\title{
Line parameters including temperature dependences of air- and self-broadened line shapes of ${ }^{12} \mathrm{C}^{16} \mathrm{O}_{2}: 2.06-\mu \mathrm{m}$ region
}

D. Chris Benner ${ }^{\mathrm{a}}$, V. Malathy Devi ${ }^{\mathrm{a}^{*}}$, Keeyoon Sung ${ }^{\mathrm{b}}$, Linda R. Brown ${ }^{\mathrm{b}}$, Charles E. Miller ${ }^{\mathrm{b}}$, Vivienne H. Payne ${ }^{b}$, Brian J. Drouin ${ }^{b}$, Shanshan $\mathrm{Yu}^{\mathrm{b}}$, Timothy J. Crawford ${ }^{\mathrm{b}}$, Arlan W. Mantz ${ }^{\mathrm{c}}$, Mary Ann H. Smith ${ }^{\text {, }}$ Robert R. Gamache ${ }^{\mathrm{e}}$

${ }^{a}$ Department of Physics, The College of William and Mary, Box 8795, Williamsburg, VA 23197, USA

${ }^{b}$ Jet Propulsion laboratory, California Institute of Technology, 4800 Oak Grove Drive, Pasadena, CA 91109, USA

${ }^{c}$ Department of Physics, Astronomy and Geophysics, Connecticut College,

New London, CT 06320, USA

${ }^{d}$ Science Directorate, NASA Langley Research Center, Hampton, VA 23681, USA

${ }^{e}$ Office of the Academic Affairs, Student Affairs, International Relations, University of Massachusetts, One Beacon Street, Boston, MA 02108, USA

Number of pages: 55

Number of Figures: 20

Number of Tables: 9

Supplemental files: 4

${ }^{a}$ Corresponding author.

Dr. V. Malathy Devi

Phone: 757.864 .5521

Fax: 757-864-4343

e mail: Malathy.d.venkataraman@nasa.gov 


\begin{abstract}
This study reports the results from analyzing a number of high resolution, high signal-to-noise ratio $(\mathrm{S} / \mathrm{N})$ spectra in the $2.06-\mu \mathrm{m}$ spectral region for pure $\mathrm{CO}_{2}$ and mixtures of $\mathrm{CO}_{2}$ in dry air. A multispectrum nonlinear least squares curve fitting technique has been used to retrieve the various spectral line parameters. The dataset includes 27 spectra: ten pure $\mathrm{CO}_{2}$, two $99 \%{ }^{13} \mathrm{C}$ enriched $\mathrm{CO}_{2}$ and fifteen spectra of mixtures of ${ }^{12} \mathrm{C}$-enriched $\mathrm{CO}_{2}$ in dry air. The spectra were recorded at various gas sample temperatures between 170 and $297 \mathrm{~K}$. The absorption path lengths range from 0.347 to $49 \mathrm{~m}$. The sample pressures for the pure $\mathrm{CO}_{2}$ spectra varied from 1.1 to 594 Torr; for the two ${ }^{13} \mathrm{CO}_{2}$ spectra the pressures were $\sim 10$ and 146 Torr. For the airbroadened spectra, the pressures of the gas mixtures varied between 200 and 711 Torr with $\mathrm{CO}_{2}$ volume mixing ratios ranging from 0.014 to $0.203 \%$. The multispectrum fitting technique was applied to fit simultaneously all these spectra to retrieve consistent set of line positions, intensities, and line shape parameters including their temperature dependences; for this, the Voigt line shape was modified to include line mixing (via the relaxation matrix formalism) and quadratic speed dependence. The new results are compared to select published values, including recent $a b$ initio calculations. These results are required to retrieve the column averaged dry air mole fraction $\left(\mathrm{X}_{\mathrm{CO} 2}\right)$ from space-based observations, such as the Orbiting Carbon Observatory-2 (OCO-2) satellite mission that NASA launched in July 2014.
\end{abstract}

Key words: $\mathrm{CO}_{2}$; Lorentz widths; pressure shifts; temperature dependences; spectral line shapes; relaxation matrix element coefficients; speed dependence 


\section{Introduction}

The 2.06- $\mu \mathrm{m}$ spectral region is used extensively for remote sensing of atmospheric carbon dioxide $\left(\mathrm{CO}_{2}\right)$ column mixing ratios [1-10]. $\mathrm{CO}_{2}$ IS BOTH LONG-LIVED AND WELL MIXED THROUGHOUT THE EARTH'S ATMOSPHERE, BUT CONCENTRATIONS OF THIS GAS IS CONTINUALLY INCREASING IN THE ATMOSPHERE, IN TURN PRODUCING GLOBAL WARMing. Climate MODEling REQUiRes PRECISE KNOWLEDGE OF THE $\mathrm{CO}_{2}$ CONCENTRATIONS AT SOURCES AND SINKS FOR THE ATMOSPHERE. Satellite missions such as the Orbiting Carbon Observatory (OCO-2) and the Greenhouse Gases Observing Satellite (GOSAT) rely on reflected sunlight in the near-infrared region to obtain columnaverage mixing ratios of $\mathrm{CO}_{2}$. WITHIN SPATIAL SCALES OF 1000 KILOMETERS, THE TYPICAL VARIATIONS ARE NO LARGER THAN 0.25\% (1 PART PER MILLION (PPM) OUT OF THE AMBIENT 400 PPM BACKGROUND). THIS PLACES STRINGENT REQUIREMENTS ON THE PRECISION AND ACCURACY OF SPACE-BORNE $\mathrm{CO}_{2}$ MEASUREMENTS, WHICH IN TURN PLACES STRINGENT REQUIREMENTS ON THE PRECISION AND ACCURACY OF THE SPECTROSCOPIC LINE PARAMETERS USED IN THE RETRIEVALS. The present study (4700-4930 $\mathrm{cm}^{-1}$ ) focuses on our efforts to provide the best line parameters for $\mathrm{CO}_{2}$ in the 2.06$\mu \mathrm{m}$ region.

During the past several years, many research groups have reported high-resolution measurements for the $2-\mu \mathrm{m}$ region of $\mathrm{CO}_{2}$ (e.g., [11-24]) including a number of line shape parameters (e.g., Lorentz half-widths and pressure-shift coefficients). In Table 1 are shown recent line shape measurements obtained for the $2.06-\mu \mathrm{m}{ }^{12} \mathrm{C}^{16} \mathrm{O}_{2}$ band, $20013 \leftarrow 00001$ [12,13,18-23]. The most comprehensive measurements by Toth et al. [11-15] included line center positions, intensities (and band strengths) and self- and air-broadened half-width coefficients. Those studies reported measurements of hundreds of $\mathrm{CO}_{2}$ bands covering the $4550-7000 \mathrm{~cm}^{-1}$ spectral region $(2.2$ $-1.43-\mu \mathrm{m}$ ), including the most abundant ${ }^{12} \mathrm{C}^{16} \mathrm{O}_{2}$ species, as well as several rarer isotopologues such as ${ }^{13} \mathrm{C}^{16} \mathrm{O}_{2}$ and ${ }^{12} \mathrm{C}^{16} \mathrm{O}^{18} \mathrm{O}$. However, these line shape studies considered neither line mixing nor speed dependence, both of which are important for atmospheric monitoring in the 2.06- to $2.0-\mu \mathrm{m}$ region (e.g., $[\mathbf{6}, \mathbf{2 5}])$. In addition, these studies used laboratory data obtained only at room temperature. 
In general, the temperature dependences of line shape coefficients are not well characterized for $\mathrm{CO}_{2}$. Suarez and Valero [17] measured temperature dependence exponents of self-broadened

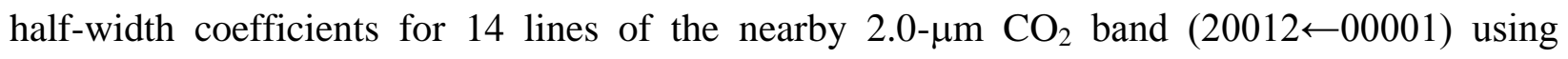
spectra obtained with a BOMEM FTS, but these few values did not fulfill the need for a comprehensive, self-consistent set of spectral line parameters for remote sensing of atmospheric $\mathrm{CO}_{2}$ in the 2.06- $\mu \mathrm{m}$ region. In our previous work [16], the multispectrum fitting approach applied to only room temperature spectra derived a self-consistent set of spectral line parameters using a quadratic speed-dependent Voigt line shape and nearest neighbor line-mixing coefficients using the off-diagonal relaxation matrix formalism [26]. These parameters were validated against atmospheric spectra from a ground-based Fourier transform spectrometer (FTS) from the Total Carbon Column Observing Network (TCCON) [6,9,10]. However, the temperature dependence of line shape parameters remained an outstanding source of uncertainty.

In addition to the line shape studies listed in Table 1, there have been a number of new measurements employing different experimental techniques concerning the 2 - $\mu \mathrm{m}$ spectral region [e.g., 27,28]. While valuable, these do not provide all the parameters needed for atmospheric

remote sensing. We began a new study of this region in 2007 and presented preliminary results at a conference [16] but later expanded our analysis to include more high-quality data (acquired with the Bruker IFS-125 HR FTS at JPL) to investigate the temperature dependence of line shapes using a wider range of optical densities.

\section{Table 1 comes here (Prior studies)}

We employed our multispectrum fitting approach $[29,30]$ to simultaneously analyze a large number of spectra [27] in order to minimize the random errors in the fit residuals and to reduce correlations between fitted line parameters. The precise results presented in this study represent the first extensive and most precise measurements of spectral line parameters (including the temperature dependence of line shapes) for $\mathrm{CO}_{2}$ and $\mathrm{CO}_{2}+$ air at 2.06- $\mu \mathrm{m}$. Our results are directly applicable to space-borne $\mathrm{CO}_{2}$ retrievals from missions such as OCO-2. 


\section{Experimental Details}

\subsection{Instrument setups, summary of experimental conditions}

Spectra used in the present analysis were recorded using two different spectrometers: 1) McMath-Pierce Fourier transform spectrometer (FTS) facility at the National Solar Observatory (NSO) on Kitt Peak, Arizona, and 2) the Bruker IFS-125HR FTS located at the Jet Propulsion Laboratory (JPL), California Institute of Technology at Pasadena, California. The experimental setups and the system configuration details are listed in Table 2. The fitted spectra were selectively chosen from a larger dataset, in order to judiciously select gas sample pressure and temperature ranges that would best determine the temperature dependences of the line shape parameters. Twenty-seven spectra obtained with seven different absorption cells were included in the final fit. Five of these were recorded at Kitt Peak, while the other twenty-two were recorded at JPL. A summary of the experimental conditions of spectra analyzed is given in Table 3.

\section{Insert Table 2 (Experimental setups)}

The spectra recorded at Kitt Peak were obtained at $\sim 0.01 \mathrm{~cm}^{-1}$ unapodized resolution. The Kitt Peak FTS was configured with a quartz halogen source, two liquid nitrogen cooled InSb detectors and $\mathrm{a} \mathrm{CaF}_{2}$ beam splitter. The experimental setup for spectra recorded at JPL consisted of a tungsten lamp for the source, a $\mathrm{CaF}_{2}$ beam splitter and an $\mathrm{InSb}$ detector. The unapodized resolutions for the 22 spectra varied between $\sim 0.004$ and $0.011 \mathrm{~cm}^{-1}$. The absorption path lengths for the 22 spectra recorded at JPL range from $0.2038 \mathrm{~m}$ to $29.3 \mathrm{~m}$. For the Kitt Peak data, a natural sample of $\mathrm{CO}_{2}$ was used for three of the spectra while a $99 \%{ }^{13} \mathrm{C}$-enriched $\mathrm{CO}_{2}$ was used for the other two. The absorption path length of the cells varied between $0.347 \mathrm{~m}$ and 49.0 $\mathrm{m}$. For the majority of the JPL (0.2038 and $20.941 \mathrm{~m}$ cells) spectra, $99.99 \%{ }^{12} \mathrm{C}$-enriched $\mathrm{CO}_{2}$ samples were used for self and air-broadening while $99.98 \%{ }^{12} \mathrm{C}$-enriched $\mathrm{CO}_{2}$ sample was used for the air-broadened spectra obtained with a room temperature multipass cell set to $29.30 \mathrm{~m}$ cell.

Table 3 comes here 
For the Kitt Peak FTS data, the $49 \mathrm{~m}$ path was obtained using the 6-m base path White cell. The $0.347 \mathrm{~m}$ and the $2.46 \mathrm{~m}$ cells were newly built to be efficient thermally-insulated chambers such that the temperature sensors could be kept in direct contact with the gas samples. Each spectrum covered the region from 3800 to $7200 \mathrm{~cm}^{-1}$. Spectrum \#1 (Table 3), with the largest path length $(49 \mathrm{~m})$ with 80 Torr $\mathrm{CO}_{2}$ included in the fit, was particularly useful in determining and validating the abundances of various rarer isotopologues in the JPL data.

For the JPL Bruker spectra, the $29.30 \mathrm{~m}$ path cell was used to obtain room temperature airbroadened spectra. The $0.2038 \mathrm{~m}$ straight path cell and the $20.941 \mathrm{~m}$ Herriott cell were both coolable and used for recording self-broadened and air-broadened spectra $(\sim 170-250 \mathrm{~K})$, respectively. The design, construction and the performance details for the two cells are detailed in Sung et al. [31] and Mantz et al. [32], respectively. For the air-broadened spectra recorded at JPL (using the $20.941 \mathrm{~m}$ Herriott cell) NIST standard air sample was used, while synthetic dry air was used for all other Bruker spectra (i.e., $0.2038 \mathrm{~m}$ and the $29.3 \mathrm{~m}$ long cell). $99.98 \%{ }^{12} \mathrm{C}$ enriched sample of $\mathrm{CO}_{2}$ was used for the $29.3 \mathrm{~m}$ spectra, while the purity of the $\mathrm{CO}_{2}$ sample was $99.99 \%$ for spectra recorded with both the coolable cells $(0.2038 \mathrm{~m}$ and $20.941 \mathrm{~m})$. More details about the experimental setups are given in Table 2.

\subsection{Calibrations of wavenumber scales}

The calibration of the wavenumber scales of the Kitt Peak spectra was determined by placing a second cell in the optical beam so that $\mathrm{CO}$ and/or $\mathrm{C}_{2} \mathrm{H}_{2}$ line positions could be used as standards $[\mathbf{3 3}, \mathbf{3 4}]$. This approach has been described in several of our previous studies (e.g., [35]). Calibration correction factors for the Bruker data were determined relative to the $2 \leftarrow 0$ band of $\mathrm{HCl}$ line positions from the HITRAN2008 database [36]. It was important that these two sets of calibration factors provided good internal consistency between both sets of spectra (Kitt Peak FTS and JPL Bruker). The multispectrum fitting software [29] used here provides the capability to adjust the wavenumber scales, interactively, if appropriate. This capability was applied for the two separate sets of data obtained with both coolable cells (see Table 3). 
In order to maintain good knowledge and control of the experimental conditions, sample pressures and temperatures were continually monitored during the entire data acquisition process. For the Kitt Peak data, gas sample pressures were monitored using calibrated capacitance manometers of appropriate pressure ranges. For the $49 \mathrm{~m}$ White cell, sample temperatures were determined using thermocouples attached to three different places on the outside surface of cell. For the shorter path cells, temperatures were determined using high accuracy $( \pm 0.05 \mathrm{~K})$ platinum resistance thermometers (Hart Scientific) inserted into the cell at four different positions (temperature sensors installed inside the cells), and these recorded temperatures varied by $\pm 0.2 \mathrm{~K}$ at most during the data acquisition period. For recording the Bruker spectra, the pressure gauges were periodically calibrated and the recommended calibration factors from the manufacturers were applied to the temperature sensors. The coolable cells used in recording the Bruker spectra are specially built with very good temperature stability and homogeneity across the length of the cells. Further details on the experimental precautions followed for the Kitt Peak spectra are listed in Miller et al. [35], while details on monitoring the gas pressures and temperatures for the data taken with the two coolable cells at JPL are described in Sung et al. [31] and Mantz et al. [32].

\section{Analysis and data retrievals}

\subsection{General procedures and equations applied}

As was done in several of our previous studies on linear molecules, such as $\mathrm{CO}_{2}$ and $\mathrm{CO}$ [e.g.,35,37,38], we followed the whole band retrieval technique originally applied by Shaw and co-workers [39] and Lin and Shaw [40], and later developed and expanded extensively by Benner et al. [29] using a better formulation of the Voigt profile [30]. Rather than adjusting the transition positions on a line-by-line basis, the multispectrum retrieval software was modified to solve for the values of the ro-vibrational constants $G, B, D$ and $H$ for the upper and lower state energies (where possible lower state constants were constrained to the best literature values). For line intensities, the vibrational band strength $S_{\mathrm{v}}$ and the Herman-Wallis factor $F$ defined by the parameters $a_{1}, a_{2}, a_{3}$ and $a_{4}$ (given below) were adjusted instead of individual line intensities. Thus our individual "measured $\square$ positions and "measured" intensities are the constrained values calculated with Eqs. (1-3). 


$$
\begin{gathered}
v_{i}=G^{\prime}-G^{\prime \prime}+\left(B^{\prime} J^{\prime}\left[J^{\prime}+1\right]-D^{\prime}\left\{J^{\prime}\left[J^{\prime}+1\right]\right\}^{2}+H^{\prime}\left\{J^{\prime}\left[J^{\prime}+1\right]\right\}^{3}\right) \\
-\left(B^{\prime \prime} J^{\prime \prime}\left[J^{\prime \prime}+1\right]-D^{\prime \prime}\left\{J^{\prime \prime}\left[J^{\prime \prime}+1\right]\right\}^{2}+H^{\prime \prime}\left\{J^{\prime \prime}\left[J^{\prime \prime}+1\right]\right\}^{3}\right) \\
S_{i}=\frac{S_{v} v_{i} L_{i} F}{Q_{r} v_{0}} \exp \left(\frac{-C_{2} E^{\prime \prime}}{T_{0}}\right)\left[1-\exp \left(\frac{-C_{2} v_{i}}{T_{0}}\right)\right]
\end{gathered}
$$

where,

$$
F=\left[1+a_{1} m+a_{2} m^{2}+a_{3} m^{3}+a_{4} J(J+1)\right]^{2}
$$

and $m=-J^{\prime \prime}$ for P-branch and $J^{\prime \prime}+1$ for R-branch lines. $v_{0}$ is the band center (equal to $G^{\prime}-G^{\prime \prime}$ ), $v_{\mathrm{i}}$ and $S_{\mathrm{i}}$ correspond, respectively, to the transition wavenumber $\left(\mathrm{cm}^{-1}\right)$ and the line intensity $\left(\mathrm{cm} /(\right.$ molecule $)$ of the $i^{\text {th }}$ line, where prime and double prime refer to the upper and lower levels; $J$ is the rotational quantum number and $Q_{\mathrm{r}}$, the rotational quantum partition function.

For the present study, the lower state ro-vibrational constants in Eq. (1) are constrained to the values from Miller and Brown [41] for ${ }^{12} \mathrm{C}^{16} \mathrm{O}_{2}$ and Elliot et al. [42] for ${ }^{16} \mathrm{O}^{12} \mathrm{C}^{18} \mathrm{O}$ and ${ }^{16} \mathrm{O}^{12} \mathrm{C}^{17} \mathrm{O}$. In Eq. (2), $E^{\prime \prime}$ is the lower state energy of the transition, and $T_{0}$ is the reference temperature $(296 \mathrm{~K}) . L_{\mathrm{i}}$ are the Hönl-London factors, $S_{\mathrm{v}}$ is the vibrational band intensity (cm/molecule), and $C_{2}$ is the second radiation constant. The values of the rotational quantum partition function, $Q_{\mathrm{r}}$, at $\mathrm{T}=296 \mathrm{~K}$ are 263.87063 for ${ }^{12} \mathrm{C}^{16} \mathrm{O}_{2}, 527.71608$ for ${ }^{13} \mathrm{C}^{16} \mathrm{O}_{2}$ and 559.30454 for ${ }^{12} \mathrm{C}^{16} \mathrm{O}^{18} \mathrm{O}[\mathbf{4 3}]$. The term $a_{4} J(J+1)$ in Eq. (3) is indistinguishable from the $a_{2}$ term in the P- and R-branches, so for bands without $\mathrm{Q}$ branches, the reported $a_{2}$ is actually very nearly $a_{2}+a_{4}$

Uncertainties are minimized with constraints in the multispectrum retrievals (1) by fitting the entire band and fitting all spectra simultaneously, (2) by applying constraints using theoretical quantum mechanical equations (eigenfunction expressions Eqs. (1) and (2)) for line positions and intensities of all major (stronger) bands, (3) by adjusting Lorentz half-widths, pressure shifts, offdiagonal relaxation matrix element coefficients and speed dependence for each line separately, (4) by including all absorption features within the fitted interval (e.g., hot bands, weak bands, and isotopologue bands), and (5) by using line mixing (relaxation matrix element coefficients) and quadratic speed dependence parameter when required as judged from fit residuals. 
The size of the fitted interval, number of spectra and the resolution of each spectrum were carefully chosen for this analysis. In the fitted interval $4700-4930 \mathrm{~cm}^{-1}$ with 27 spectra, absorption features were observed from 42 vibrational bands (24, 9, 2, 3, and 4) belonging respectively, to ${ }^{12} \mathrm{C}^{16} \mathrm{O}_{2},{ }^{13} \mathrm{C}^{16} \mathrm{O}_{2},{ }^{12} \mathrm{C}^{16} \mathrm{O}^{18} \mathrm{O},{ }^{12} \mathrm{C}^{16} \mathrm{O}^{17} \mathrm{O}$ and ${ }^{13} \mathrm{C}^{16} \mathrm{O}^{18} \mathrm{O}$. For several very weak bands, their parameters were held fixed to predicted values from HITRAN2004 [44] (see the Supplemental file discussed later). Other very weak bands belonging to the rarer isotopologues (e.g., ${ }^{18} \mathrm{O}$ ) were not significant, and no effort was made to obtain their line parameters. In all, 21 of 42 bands were measured in the fitting. The band identifications with their upper and lower vibrational levels, range of quantum numbers of transitions fitted in the $\mathrm{P}, \mathrm{Q}$ (when present), and $\mathrm{R}$ branches, and the isotopologue identifications for these 21 bands are listed in Table 4.

\section{Table 4 appears here (major bands included in the analysis)}

\subsection{Constraints}

The least squares fittings were initialized using the line list from HITRAN2004 [44]. The final input file contained 3040 spectral lines from the initial line list used in [16] from HITRAN2004 with additional lines (e.g., for ${ }^{12} \mathrm{C}^{16} \mathrm{O}^{18} \mathrm{O}$ ) added later from Toth et al. [14] to include all observable features in the $4700-4930 \mathrm{~cm}^{-1}$ region of the fitted spectra. In order to extract the most information for the stronger bands, the least squares fit solves for the ro-vibrational constants; for this the positions were calculated from the ro-vibrational constants extending to the highest $J$ for which position constraints are written and is the same as in HITRAN [44]. When two different bands involve the same vibrational state, there is a further reduction in the number of degrees of freedom in the solution since those bands will access some of the same ro-vibrational constants. Similarly the individual line intensities are calculated from the vibrational band strength $\left(S_{\mathrm{v}}\right)$ and Herman-Wallis-type parameters measured in this study along with the known quantum numbers. The multispectrum fit then solves for only the vibrational band strength $\left(S_{\mathrm{v}}\right)$ and the Herman-Wallis parameters for each band. Positions and intensities of several of the stronger bands are also constrained. For the very weak bands, the line positions and intensities are either fixed to the initial values assumed from HITRAN 2004 [44] or the values of Toth et al. $[\mathbf{1 1}, \mathbf{1 4}, \mathbf{1 5}]$, since the analysis of the present study started in 2006 was initially begun with only 
room temperature spectra [16]. Positions and intensities for a number of weak transitions (where no constraints were setup) were also adjusted during the fittings, as judged from the fit residuals.

\subsection{Sample spectra and multispectrum fits}

As example, a set of (unfitted) experimental spectra included in the present analysis is shown in Fig. 1.

\section{Insert Fig. 1 here}

Fig. 1(a-e) shows the signal levels $\left(0-100 \%\right.$ scale) vs. wavenumber $\left(\mathrm{cm}^{-1}\right)$ for 5 of the 27 spectra analyzed in this work. The spectra plotted in the top two panels (a) and (b) represent majority of the transitions observed in the entire fitted region. Panel (a) represents the spectrum with the longest absorption path length of $49 \mathrm{~m}$ in the dataset, with $\mathrm{CO}_{2}$ at natural isotopologue abundance with 80 Torr and at room temperature, while panel (b) represents one of the (only) two spectra with the highest isotopologue abundance of $\left(99 \%{ }^{13} \mathrm{C}\right.$-enriched $){ }^{13} \mathrm{CO}_{2}$. It is clear that the ${ }^{13} \mathrm{CO}_{2}$ features in (b) are practically absent in panels (c-d) but appear in (a). The ${ }^{12} \mathrm{C}^{16} \mathrm{O}^{18} \mathrm{O}$ bands appear in (a) and (c), even though data in (c) were obtained with a $99.99 \%{ }^{12} \mathrm{C}$-enriched carbon dioxide. The first hot band $(21113 \leftarrow 01101)$ transitions of ${ }^{12} \mathrm{C}^{16} \mathrm{O}_{2}$ centered at 4807.694 $\mathrm{cm}^{-1}$ are seen in (a), (c) and (d). In the bottom panel (e), most of the transitions are from the strongest $20013 \leftarrow 00001$ band of ${ }^{12} \mathrm{C}^{16} \mathrm{O}_{2}$ located at $4853.523 \mathrm{~cm}^{-1}$, because the other weaker bands almost disappear when the sample is cooled to $\sim 170 \mathrm{~K}$. Weak absorptions from the $20013 \leftarrow 00001$ band of ${ }^{12} \mathrm{C}^{16} \mathrm{O}^{18} \mathrm{O}$ are still visible near $4910 \mathrm{~cm}^{-1}$. In fitting all 27 spectra, the abundances for each of the isotopologues in every spectrum must be determined accurately enough such that when fitted simultaneously, the residuals are reduced to the noise levels of the spectra as was done in the present study (PS).

The final multispectrum fit of all 27 spectra is plotted in Fig. 2. The top panel (a) shows the observed spectra, and the bottom panel (b) shows the global weighted observed minus calculated (O-C) fit residuals.

Insert Fig.2 here 
Some small but persistent residuals (corresponding to the $20013 \leftarrow 00001$ band of ${ }^{12} \mathrm{C}^{16} \mathrm{O}_{2}$ around $4850 \mathrm{~cm}^{-1}$ ) are still present, as seen in Fig. 2(b). The sources of these residuals are not understood at this time, but they could be due to line mixing from non-neighboring lines not included in this study, missing transitions from rarer isotopologues or other causes of unknown origin. Fig. 3 shows the fit residuals grouped by temperature ranges (from the multispectrum fit). Persistent residual features are not obvious in the ${ }^{13} \mathrm{C}^{16} \mathrm{O}_{2}$ spectrum (room temperature spectrum and not high enough pressure to have line mixing) in (c), but they are clearly visible in the cold temperature residuals in (d) and (e). Spectra plotted in Figs. 2 and $\mathbf{3}$ have been given weights in accordance with their different signal-to-noise ratios. The largest weighted residual anywhere on the fit for any spectrum is $<0.2 \%$. The standard deviation of the fit for unit weight (the highest signal-to-noise ratio spectra in the solution) was $0.037 \%$.

\section{Insert Fig. 3 here}

\subsection{Lorentz half-width and pressure-shift coefficients}

The Lorentz half-width coefficients, pressure-shift coefficients, and their temperature dependences were measured on a line-by-line basis and defined by Eqs. (4-6) given below [38]:

$$
\begin{aligned}
& b_{L}=p \times\left[b_{L, a i r}^{0} \times(1-\chi)\left(\frac{T_{0}}{T}\right)^{n_{1}}+b_{L, \text { self }}^{0} \times \chi\left(\frac{T_{0}}{T}\right)^{n_{2}}\right] \\
& \nu=v_{0}+p \times\left[\delta_{\text {air }} \times(1-\chi)+\delta_{\text {self }} \times \chi\right] \\
& \delta=\delta^{0}+\delta^{\prime} \times\left(T-T_{0}\right)
\end{aligned}
$$

In Eqs. (4-6), $b_{L}^{0}$ and $\delta^{0}$ represent pressure broadening and pressure-shift coefficients (in $\mathrm{cm}^{-1}$ atm $^{-1}$ at $296 \mathrm{~K}$ ), respectively. $b_{\mathrm{L}}$ is the Lorentz half-width (in $\mathrm{cm}^{-1} / \mathrm{atm}$ ) of the spectral line at pressure $p$ and temperature $T, b_{L, a i r}^{0}$ and $b_{L, \text { self }}^{0}$ are the Lorentz half-width coefficient of the line for air- and self-broadening, respectively, at the reference pressure $p_{0}(1 \mathrm{~atm})$ and temperature $T_{0}$ $(296 \mathrm{~K})$, and $\chi$ is the ratio of the partial pressure of $\mathrm{CO}_{2}$ to the total sample pressure in the cell. Similar to air- and self-broadened width and shift coefficients, temperature dependences of airand self-broadened half-width and pressure-shift coefficients were also measured individually for each transition. As shown in Eq. (6) a linear model was used for the temperature dependence of pressure-shift coefficients. 
Applying the above expressions, Lorentz half-width and pressure-shift coefficients have been measured for a few hundred individual transitions. Where ro-vibrational constants were measured rather than individual line positions, the pressure-shift coefficients were well determined because of the more precise zero-pressure line position retrievals and the large reduction of correlation between the zero-pressure line positions and the pressure-shift coefficients. Similarly, correlations were also reduced between the line intensities and the Lorentz half-width coefficients which are characteristics for strong transitions due to the constraints upon the line intensities.

The measured line parameters and number of $\mathrm{CO}_{2}$ measurements from this study are provided in Table 5.

\section{Table 5 comes here}

The number of positions and intensity measurements given under columns 2 and 4 (first row) lists two values: the top number corresponds to constrained values while the number beneath (within parentheses) correspond to the total number of measured positions and intensities including those that were retrieved as part of the constrained parameters (top row). The number of measured intensities in Table $\mathbf{5}$ is slightly greater than the number of measured positions because those positions greatly blended with other lines are excluded in the count. Generally, more self-broadening and self-shift coefficients are measured than air-broadening and air-shift coefficients since there are higher carbon dioxide amounts in pure sample data than for the airbroadened spectra. Further details about each measured parameter will be discussed in the following sections.

\section{Discussion of results and comparison with databases}

The $\mathrm{CO}_{2}$ line parameters have undergone several improvements/modifications in the last three editions of the HITRAN databases [36,44,45]. For HITRAN2004 [44], values from the Direct Numerical Diagonalization (DND) technique [46] were replaced by combining selected experimental results with calculated line positions and intensities from the 2003 version of the 
Carbon Dioxide Spectroscopic Databank, CDSD-1000 [47]. The line parameters compilation by Toth et al. in [15] was used for further improvements in the positions and intensities for HITRAN2008 [36]. In HITRAN2012 [45], the database has been greatly expanded and improved using an updated CDSD-296 databank [48] that includes line parameters for $12 \mathrm{CO}_{2}$ isotopologues providing spectroscopic information not only for positions and intensities but also for air- and self-broadened Lorentz half-width coefficients, temperature dependences of airbroadened half-width coefficients and air- pressure-induced shift coefficients. The CDSD-296 [48] line list includes $\mathrm{CO}_{2}$ transitions with intensities as low as $\sim 1 \times 10^{-30} \mathrm{~cm}^{-1} /\left(\right.$ molecule $\left.\mathrm{cm}^{-2}\right)$ at $296 \mathrm{~K}$. Comparisons of present line parameters with the CDSD-296 [48] are shown in several graphs and discussed in the section describing estimated total error budget. In addition to the CDSD-296 [48] databank, there are two other sources of line parameters that are theoretically computed $[49,50]$ with which present results are compared. In the following subsections we detail how our new ${ }^{12} \mathrm{CO}_{2}$ measurements for the $20013 \leftarrow 00001$ band compare with HITRAN values, selected laboratory measurements [e.g., Refs. 11-13,] and recent theoretical calculations $[48-50]$.

\subsection{Line positions}

Detailed descriptions of constraints and the theoretical quantum mechanical expressions used for determining the line positions were provided in Section 3.1. Line positions from PS are compared to values reported in the HITRAN databases $[\mathbf{3 6}, \mathbf{4 4 , 4 5}$ ] and Toth et al. [11] for the $20013 \leftarrow 00001$ band of ${ }^{12} \mathrm{C}^{16} \mathrm{O}_{2}$ and are plotted in Fig. 4. Since the initial line list used in our earlier analysis of room temperature spectra [16] was from HITRAN2004 [44], values from HITRAN2004, HITRAN2008 [36], and the more recent HITRAN2012 [45] compilations are included in the comparisons. The means and standard deviations of the position differences between PS and $[\mathbf{1 1}, \mathbf{3 6}, \mathbf{4 4}, \mathbf{4 5}]$ are given in the plot. The mean difference PS-Toth et al. [11] shows the smallest value.

\section{Insert Fig. 4 here}

The position differences between PS and HITRAN $[\mathbf{3 6}, \mathbf{4 4 , 4 5}]$ are larger than the difference between this work and Toth et al. [11] because Toth et al. measured positions for P58-R58 only 
while the constrained positions from PS extend up to $J^{\prime \prime}=84$. The position differences between PS and HITRAN2004 [44] are slightly smaller compared to the differences between PS and HITRAN [36,45]. Positions and uncertainties extrapolated to higher- $J\left(J^{\prime \prime}>70\right)$ in the present study assume that the ro-vibrational model used here is correct, which is likely to be close to the truth for the $20013 \leftarrow 00001$ band of ${ }^{12} \mathrm{C}^{16} \mathrm{O}_{2}$. However, a few $\%$ difference exists between the vibrational band strength $\left(S_{\mathrm{v}}\right)$ and the total integrated band strength $\left(S_{\text {band }}\right)$ that is attributed to small vibration-rotation interactions, as discussed in a later section. The constrained positions also depend upon the ro-vibrational constants for both the upper state (determined from PS) and the lower state (fixed to literature values given in Table 6(a)). If line positions from PS are compared to only P58-R58 transitions corresponding to [11], the mean and standard deviation for all the four comparisons shown in Fig. 4 would be $0.0001716(8) \mathrm{cm}^{-1}$.

\subsection{Line Intensities}

Fig. 5 displays the measured line intensities vs. $m$ along with uncertainties for the $20013 \longleftarrow 00001$ band of ${ }^{12} \mathrm{C}^{16} \mathrm{O}_{2}$. The uncertainties shown in (b) assume that the model used for constraining the intensities is correct. This is likely to be true as long as there are no unrecognized local or nonlocal resonances for unobserved lines at high $J$ (i.e., $J^{\prime \prime} \geq=70$ ). The advantage of constraining the intensities to theory is that the uncertainties remain small at high $J$ even when the lines are disappearing in the data (especially at cold temperatures). At $J=80$ the internal uncertainties are about $0.1 \%$ (discussed and shown in a later figure). However, the sample pressures, temperatures, and absorption path lengths are only known to this level of uncertainty at best, so the absolute error in intensities of all lines plotted could be several tenths of a percent to one percent. Further details on absolute error estimates are given in the discussion section.

\section{Insert Fig. 5 here}

The retrieved line intensities for the strongest ${ }^{12} \mathrm{C}^{16} \mathrm{O}_{2}$ band, $20013 \leftarrow 00001$, are plotted vs. $m$ in Fig. 5 on both a linear (5a) and a logarithmic (5b) scale. The corresponding intensity uncertainties are also plotted in (5b). The PS intensities are compared to HITRAN2004 [44], HITRAN2008 [36] and HITRAN2012 [45] as well as to Toth et al. [11]. Similar to line positions, line intensities for this band (along with hundreds of other $\mathrm{CO}_{2}$ bands) from Toth et al. 
$[\mathbf{1 1}, \mathbf{1 5}]$ were used in $[\mathbf{3 6 , 4 5}]$. As seen in Fig. 5(b), the uncertainties in intensities are $J$-dependent since both intensities and their uncertainties are constrained to follow Eqs. (2) and (3).

The ratios of line intensities between PS and HITRAN2012 [45] and PS and Toth et al. [11] are plotted in Fig. 5(c). The comparison between PS and Toth et al. [11] is limited to measured transitions listed in [11], while the comparison with HITRAN2012 applies to all constrained line intensities $\left(J^{\prime \prime}=84\right)$ and even higher $J$. The ratios between PS and HITRAN2004 showing the largest variability are also included since the intensities in our initial room temperature fittings [16] were from [44]. The line intensities in $[\mathbf{3 6 , 4 5}]$ for the P58-R58 are nearly the same $[\mathbf{1 1}]$ and hence indistinguishable in Fig. 5(c). The reason for the discontinuity observed near $\boldsymbol{m}=58$ and 60 [45] is likely associated with some small discrepancies while combining intensity files from different sources (see Refs. [45,48] for details). Although Toth et al. [11] used a Voigt line shape model and performed a spectrum-by-spectrum analysis, the mean ratios of intensities (PS/Toth et al. $)=0.992(5)$ for P58-R58. The ratios of intensities computed between PS and Refs. $[\mathbf{3 6 , 4 4 , 4 5}]$ are given in panel 5(c). No other extensive experimental measurements of intensities have been recently reported for this band (see Table 1).

The measured ro-vibrational $(G, B, D, H)$ and the transition moment constants (vibrational band strength $S_{\mathrm{v}}$ and the Herman-Wallis terms, $a_{1}, a_{2}, a_{3}, a_{4}$ ) for several bands that were constrained to theory using Eqs. (2) and (3) are listed in Table 6(a). In the present study, the $a_{1}, a_{2}$ and $a_{3}$ terms were determined for seven of the eight bands listed in Table 6(a), and were found sufficient to describe the strongest ${ }^{12} \mathrm{C}^{16} \mathrm{O}_{2} 20013 \leftarrow 00001$ band, its hot band $21113 \leftarrow 01101$ and six other bands. For the weaker $30013 \leftarrow 10002$ band of ${ }^{12} \mathrm{C}^{16} \mathrm{O}_{2}$, only the $a_{1}$ and $a_{2}$ terms were needed to obtain a good fit. Table 6(a) also lists the correlation coefficients between appropriate pairs of the fitted parameters. These correlation coefficients are required to calculate the uncertainties of the positions and intensities of the individual lines (see the discussion section for details). These values also provide confidence in the global least squares fits. The correlation coefficients between any two unconstrained line parameters determined in this work are internally calculated in the multispectrum software and used for the uncertainty analysis. This matrix contains over 610000 entries $\{n(n-1) / 2)\}, n$ being the number of adjusted parameters in the multispectrum fit and hence is not reported here. Correlation coefficients for the upper state ro-vibrational 
constants for the $21113 \leftarrow 01101 e, 21113 \leftarrow 01101 f$ and the $40002 \leftarrow 10002$ bands (interacting bands) of ${ }^{12} \mathrm{C}^{16} \mathrm{O}_{2}$ are listed in Table $6(\mathbf{b})$.

\section{Insert Table 6(a) and 6(b) here}

Comparisons of the vibrational band strengths $(\mathrm{cm} /$ molecule at $296 \mathrm{~K})$ for a few selected bands measured in the PS with Toth et al. [11] are provided in Table 7. Only those bands whose vibrational band strengths, $S_{\mathrm{v}}$, have been determined using theoretical quantum mechanical expressions (constraints) are listed in this table. Recall that measurements in [11] were made without using constraints.

\section{Table 7 comes here}

Vibrational band strengths $\left(S_{\mathrm{v}}\right)$ for nine bands (five for the main isotopologue ${ }^{12} \mathrm{C}^{16} \mathrm{O}_{2}$, two for ${ }^{13} \mathrm{C}^{16} \mathrm{O}_{2}$ and two for ${ }^{12} \mathrm{C}^{16} \mathrm{O}^{18} \mathrm{O}$ ) are given in Table 7. The upper and lower levels for the bands with approximate band centers (for identification of bands) are also provided. For all cases, including the enriched samples, appropriate abundances for the various isotopologues for a natural carbon dioxide sample were used in determining the intensities.

Often the vibrational band strength, $S_{\mathrm{v}}$, differs from the integrated band strength, $S_{\text {band }}$, which is defined as the sum of the intensity of all lines in the band. The following discussion shows the relationship between the sum of the line intensities and the vibrational band strength and presents the results determined from present measurements whose values are listed in Tables 6 and 7.

Starting from the general expression for the intensity of a line in terms of the total transition moment squared, which in general contains all interactions (for example see Eq. (38) of Ref. [51] or Eq. (17) of Ref. [52]), the vibrational band strength can be defined as follows. Assuming no interactions, the total transition moment squared can be written as a product of a rotationless (vibrational) transition moment squared times the transition moment squared for a rigid rotor (Hönl-London factors). Further, assuming $Q_{\mathrm{tot}}=Q_{\mathrm{vib}} \times Q_{\mathrm{rot}}$, i.e., the product approximation for 
the partition sum, the line intensity expression can be written as a vibrational part times a rotational part. $S_{\mathrm{v}}$ is defined by the vibrational part,

$$
S_{v}=\frac{8 \pi^{3}}{3 h c} \nu_{\mathrm{v} ? \leftarrow \mathrm{v}}\left(\frac{e^{-C_{2} E_{\mathrm{v}} / T}}{Q_{\mathrm{v}}}\right)\left|R_{\mathrm{v} ? \leftarrow \mathrm{v}^{\prime \prime}}\right|^{2}
$$

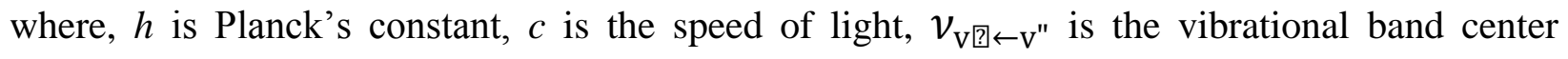
corresponding to $v_{0}$ in Eq. (2), $E_{\mathrm{v}}$ " is the lower state vibrational energy, $C_{2}$ is the second radiation constant $\left(\mathrm{cm}^{-1} \cdot \mathrm{K}\right), T$ is the temperature in Kelvin, $Q_{\mathrm{v}}$ is the vibrational partition sum, and $\mid R$ $\left.v^{\prime} \in v^{\prime \prime}\right|^{2}$ is the vibrational transition moment squared. Furthermore, if the line intensity expression is summed over all lines of a band, a sum rule can be applied reducing the rotational part to 1 giving $S_{\mathrm{v}}=S_{\text {band }}$. Note, this is only for the case for no vibration-rotation interactions. Further details are available in Ref. [52].

When vibration-rotation interactions are present, the line intensity formula is generally augmented with the Herman-Wallis factor, which defines the departure of line intensities from rigid-rotor behavior (see Eq. (2)). In such cases $S_{\mathrm{v}}$ does not equal $S_{\text {band. }}$ The difference between the two can be used as a proxy to the strength of the vibration-rotation interactions. For most parallel bands the differences are only a few percent. In the present study, the ratio of the integrated band strength, $S_{\text {band, }}$, to the vibrational band strength, $S_{\mathrm{v}}$, is 1.033 , indicating a small vibration-rotation interaction for this band. When the vibration-rotation interactions are strong, significant differences between $S_{\text {band }}$ and $S_{\mathrm{v}}$ are observed, see for example Rinsland et al. [53].

The integrated band strength $S_{\text {band }}$ for the $20013 \leftarrow 00001$ band of ${ }^{12} \mathrm{C}^{16} \mathrm{O}_{2}$ from PS is obtained by summing up the measured (or calculated) individual line intensities to be $7.110 \times 10^{-21}$ $\mathrm{cm} /$ molecule at $296 \mathrm{~K}$ and shows excellent agreement (except Ref. 44]) with the band strength values of $7.127 \times 10^{-21}[\mathbf{1 1}], 7.145 \times 10^{-21}[\mathbf{3 6}, \mathbf{4 5}], 8.042 \times 10^{-21}[\mathbf{4 4}]$ and $7.134 \times 10^{-21}[\mathbf{5 0}]$ $\mathrm{cm} /$ molecule at $296 \mathrm{~K}$. The vibrational band strength $S_{\mathrm{v}}$ for the $20013 \leftarrow 00001$ band of ${ }^{12} \mathrm{C}^{16} \mathrm{O}_{2}$ from PS obtained directly from our multispectrum least squares fittings and listed in Table 6(a) is $6.884 \times 10^{-21} \mathrm{~cm} /$ molecule at $296 \mathrm{~K}$. Thus we find that the ratio of the integrated band strength 
$S_{\text {band }}\left(7.110 \times 10^{-21} \mathrm{~cm} /\right.$ molecule $)$ to the vibrational band strength $S_{\mathrm{v}}\left(6.884 \times 10^{-21} \mathrm{~cm} / \mathrm{molecule}\right)$ for the $20013 \leftarrow 00001$ band of ${ }^{12} \mathrm{C}^{16} \mathrm{O}_{2}$ is 1.033 , indicating a small vibration-rotation interaction for this band. For the same band, a similar ratio (1.033) has been obtained between $S_{\text {band }}$ and $S_{\mathrm{v}}$ by Toth et al. [11].

\subsection{Interacting bands and Fermi resonance}

Two pairs of bands in this study have interacting levels involved in Fermi resonance. The first of these interacting pairs, the $12212 \leftarrow 00001$ and the $23301 \leftarrow 00001$ bands of ${ }^{12} \mathrm{C}^{16} \mathrm{O}_{2}$, is located near the high wavenumber limit of the fitted interval. This interaction is due to an unusual resonance where the predicted level crossing near $J=-25$ is avoided. While $J<0$ is not physically allowed, this mathematical "crossing" still affects the energy of the allowed levels for $J>0$. The interacting vibrational energies are separated by just over $2 \mathrm{~cm}^{-1}$. The $12212 \leftarrow 00001$ band is very weak; also no lines of the weaker $23301 \leftarrow 00001$ band could be identified to report the corresponding intensities. This prevented us from modeling the interaction between these bands. However, for the weak $12212 \leftarrow 00001$ band, 24 line positions and 25 line intensities between P50 and R52 were individually measured; the remaining higher $J$ line parameters were fixed to the values of the HITRAN2004 database [44].

The second interacting pair consists of a very weak $40002 \leftarrow 01101$ band which interacts with the $e$ component of the much stronger $21113 \leftarrow 01101$ band of ${ }^{12} \mathrm{C}^{16} \mathrm{O}_{2}$ (first hot band of $20013 \leftarrow 00001$ and the second strongest band in the spectral region after $20013 \leftarrow 00001$ ). Without the interaction, the $40002 \leftarrow 01101$ band would not be seen in our data. The modeling is complicated because the $21113 f$ level is not interacting and its transitions are visible in the spectrum. This prevents the solution from overly adjusting the interaction to compensate for other uncertainties in the solution. Line parameters for both interacting bands were adjusted and the unperturbed lines ( $f$ components) of the $21113 \leftarrow 01101$ band were constrained to the same position and intensity constraints as for the perturbed lines. The same interaction coefficient was forced to be used for both the positions and intensities of the two bands. Eqs. (1-3) were used to compute the unperturbed positions and intensities of all lines with only the rotation-vibrational constants, vibrational band strengths and Herman-Wallis constants varied in the solution. A 
single interaction coefficient was then used to compute the perturbed positions and intensities of the lines involved in the interaction using the formulation of Ref. [54]. The perturbed energies, $E$, of the upper levels at each $J$ were found from the two unperturbed energies, $E_{1}$ and $E_{2}$ and the interaction constant $W_{12}[\mathbf{5 5}]$.

$$
\begin{aligned}
& E=\frac{1}{2}\left(E_{1}+E_{2}\right) \pm \frac{1}{2} \sqrt{4\left|W_{12}\right|^{2}+\delta^{2}} \\
& \delta=\left(E_{1}-E_{2}\right) \\
& W_{12}=\Omega \sqrt{J J+1)}
\end{aligned}
$$

$\Omega$ is the interaction coefficient for the two bands. The perturbed intensities, $I^{p}$, of the spectral lines are computed from the unperturbed intensities, $I^{0}$, and the parameters above by the following relationships.

$$
\begin{gathered}
\sqrt{I_{1}^{p}}=\mathrm{c} \sqrt{I_{1}^{0}}-\mathrm{d} \sqrt{I_{2}^{0}} \\
\sqrt{I_{2}^{p}}=\mathrm{d} \sqrt{I_{1}^{0}}+c \sqrt{I_{2}^{0}} \\
c=\left(\frac{\sqrt{4\left|W_{12}\right|^{2}+\delta^{2}}+\delta}{2 \sqrt{4\left|W_{12}\right|^{2}+\delta^{2}}}\right)^{0.5} \\
d=\left(\frac{\sqrt{4\left|W_{12}\right|^{2}+\delta^{2}}-\delta}{2 \sqrt{4\left|W_{12}\right|^{2}+\delta^{2}}}\right)^{0.5}
\end{gathered}
$$

This interaction shares many of the features of the weaker interacting pair $(12212 \leftarrow 00001$ and the $23301 \leftarrow 00001$ bands of ${ }^{12} \mathrm{C}^{16} \mathrm{O}_{2}$ ) discussed earlier. The mathematical crossing for this interacting pair is near $J=-15$, but the separation of the upper state vibrational levels is only 0.48 $\mathrm{cm}^{-1}$ (compared to $\sim 2 \mathrm{~cm}^{-1}$ for the other weaker interacting pair). This smaller separation makes 
the interaction much stronger. The result is that up to $8 \%$ of the intensity of the stronger band is transferred to the weaker band and the positions of the lines in the two bands are moved as much as $0.15 \mathrm{~cm}^{-1}$. Fig. 6(a) displays the shift of each spectral line in position as a function of $J^{\prime}$ of this band. The shift in position starts at $J^{\prime}=0$ since the interaction coefficient is multiplied by $J^{\prime}\left(J^{\prime}+1\right)$. The shift grows rapidly due to the rapid change in $J^{\prime}\left(J^{\prime}+1\right)$, but then eventually slows and turns around near $J^{\prime}=50$ at a shift of about $0.15 \mathrm{~cm}^{-1}$ due to the increasing value of $\delta$ (separation between the unperturbed energies).

The fraction of the intensity of the stronger band borrowed by the weaker band is plotted in Fig. 6(b); the effect is zero at $J^{\prime}=0$ due to the $J^{\prime}\left(J^{\prime}+1\right)$ factor in the interaction constant. Around $J^{\prime}=15$, the intensity borrowing peaks at about $8 \%$ and then starts a slow decline due to the increasing distance from the mathematical crossing until a minimum of about $4.5 \%$ occurs near $J^{\prime}=50$. The $J^{\prime}\left(J^{\prime}+1\right)$ factor then overcomes the larger $\delta$ very slowly until a peak of about $4.5 \%$ occurs near $J^{\prime}=70$. The interaction coefficient is determined to be $0.019295(5)$ compared to $0.019300(140)$ as found by Toth et al. [11]. This few percent of intensity of the stronger band is still about 2000 times the intrinsic intensity of the weaker band (see Table 6(a)). While the effect is small (a few percent) on the stronger band, the effect on the weaker band is very large and the characterization of the interaction is crucial.

Figs. 6(a) and 6(b) come here (interacting band)

The band strength of the weaker band $(40002 \longleftarrow 01101)$ was too small to be determined in our solution, but by trial and error, an approximate value was found. The weaker band's intrinsic intensity is about 50000 times smaller than that of the stronger band (Table 6(a)), but the lines are visible because the interaction transfers significant intensity into the weaker band. Note that the interaction coefficient that affects both the intensities and positions of both bands is determined with an uncertainty of $0.03 \%$. 


\subsection{Lorentz half-width coefficients and their temperature dependences}

Numerous pressure-broadened half-width and pressure-shift coefficients were also determined from the present study. The best information on the width and shift coefficients is from spectra with large amounts of carbon dioxide and high pressures. With traditional single spectrum analysis, these spectra pose a number of difficulties. First, the broader lines are more likely to be blended, and it is difficult to retrieve reliable individual half widths. Second, for lines having larger optical depths, retrieval of the Lorentz half width is highly inversely correlated with the intensity of the line. Thus, constraining line intensities of both the strong and weak transitions to the same theoretical formula using multispectrum fitting technique provides internally consistent retrievals. With the better-determined intensities, the measured values of the individual halfwidths are more precise.

The measured air- and self-broadened Lorentz half-width coefficients, pressure-shift coefficients, and their corresponding temperature dependences along with line positions and intensities are listed in the Supplemental file (discussed later). The majority of the half-width and pressureshift coefficients were measured for the strongest band $(20013 \leftarrow 00001)$ and its first hot band (21113־01101) of ${ }^{12} \mathrm{C}^{16} \mathrm{O}_{2}$. Similar parameters could be measured only for a fewer number of transitions in other bands. Temperature dependences of half-widths, pressure-shifts and offdiagonal relaxation matrix elements were measured only for the $20013 \leftarrow 00001$ band of ${ }^{12} \mathrm{C}^{16} \mathrm{O}_{2}$.

As example, in Table 8, all measured line parameters (except the off-diagonal relaxation matrix element coefficients) for the $20013 \longleftarrow 00001$ band of ${ }^{12} \mathrm{C}^{16} \mathrm{O}_{2}$ are listed for the P20-R20 transitions. In the Supplemental file, the air- and self-broadening parameters (widths, shifts and their temperature dependences) for all measured transitions are listed under separate columns. In the sample Table 8, the line parameters for air-broadening are given at the top of each row, and the self-broadening parameters are given underneath. The identification of each transition, its position and intensity (with corresponding uncertainties) are provided. For the line intensities, half-width coefficients and their temperature dependence exponents, uncertainties in $\%$ are given in the column next to their values while the uncertainties for positions, pressure-shift coefficients and their temperature dependences are provided in parentheses corresponding to the last quoted digits. The last two columns in Table 8 list the quadratic speed dependence 
parameter for each transition and their \% uncertainties. We have assumed that the speed dependence parameter is independent of both the broadening gas $\left(\mathrm{CO}_{2}\right.$ and air in this study) and the temperature of the gas sample, hence a single value common to both $\mathrm{CO}_{2}-\mathrm{CO}_{2}$ and $\mathrm{CO}_{2}$-air broadening has been assumed. Lisak et al. [56] recently published theoretical prediction for the temperature dependence of the quadratic speed dependence parameter. Future analysis could include this model as an appropriate physical constraint on the speed-dependent parameter.

\section{Insert Table 8 here}

The values in Table 8 are representative of the high precision achieved in our multispectrum fittings (for strong, unblended lines) where the random errors are minimized to the noise levels of the spectra. All listed uncertainties correspond to one-sigma internal statistical errors in the last quoted digit(s) obtained from the multispectrum fit. The uncertainties listed above do not include any systematic errors that might arise due to unknown sources (e.g., uncertainties in the physical conditions of spectra, absorption path lengths, line shape model used).

The measured air- and self-broadened half-width coefficients for the $20013 \leftarrow 00001$ are plotted vs. $m$ ( $m=-J^{\prime \prime}$ for P-branch lines and $J^{\prime \prime}+1$ for R-branch lines) in Fig. 7, where the values are compared to those measured by Toth et al. [12,13], Régalia Jarlot et al. [18], and HITRAN2012 [45]. Even though there are no theoretical constraints to the half-width coefficients, the values still follow a fairly smooth curve; however, at high $J$, the half-width coefficients are less well determined because of the weakness of those absorptions (even in spectra with the largest gas amounts, especially at cold sample temperatures). The uncertainties are also plotted, but they are not visible in most cases since their values are smaller than the symbol size. Toth et al. [12,13] fitted their measured half-width coefficients to a model (polynomial expression in $m$ ) after measuring each value individually; as seen in Fig. 7, the trend with $J$ is smooth. The width coefficients in HITRAN2012 [45] also follow a smooth curve since those were calculated using an empirical polynomial expressions in $m$ obtained by fitting measured values. Also included are

the predicted Lorentz air- and self-broadened half-width coefficients by Gamache and Lamouroux [57] which are in very good agreement with other measurements and calculations. 


\section{Insert Fig. 7 comes here (half-width coefficients)}

The ratios of self- and air-broadened half-width coefficients from PS to Toth et al. [12,13] as a function of $m$ are plotted in Figs. 7(b) and 7(c), respectively. The ratios of self- to air-broadened half-width coefficients as a function of $m$ from PS, Toth et al. and HITRAN2012 are shown in Fig. 7(d). As expected, the self-width to air-width ratios are $m$-dependent and vary from 1.19(13) to about 1.28(10) in the three cases compared. The mean ratios from PS and Toth et al. [12,13] agree well while the mean ratio from HITRAN2012 [45] gives a smaller value, but all three ratios agree within the mutual scatter from the other two ratios.

The temperature dependence exponents for air- and self- broadened half-width coefficients $\left(n_{1}\right.$ and $n_{2}$, respectively) for the $20013 \leftarrow 00001$ band of ${ }^{12} \mathrm{C}^{16} \mathrm{O}_{2}$ have been determined in the PS. A power law model shown in Eq. (4) was applied to fit all the spectra (see Table 3) simultaneously. These power law parameters $\left(n_{1}\right.$ and $\left.n_{2}\right)$ are needed on a line-by-line basis for accurate remote sensing of $\mathrm{CO}_{2}$. The measured $n_{1}$ and $n_{2}$ are shown as a function of $m$ in Fig. 8(a). The black horizontal dashed line corresponds to the value of $n=0.75$, usually assumed when no measured values are available.

Fig. 8 comes here (T-dep. Widths)

Since no extensive measurements for temperature dependence exponents of half-width coefficients for air- or self-broadening have been published in the literature, the present values for $n_{2}$ are compared to the experimental measurements reported for the $20012 \leftarrow 00001$ band of ${ }^{12} \mathrm{C}^{16} \mathrm{O}_{2}$ by Suarez and Valero [17]. Although measurements in [17] were available only for $m=$ 6-32, the general pattern of the variation of $n_{2}$ vs. $m$ is similar to what is observed in the present work. In the PS, the variations are almost identical in the $\mathrm{P}$ and $\mathrm{R}$ branches. The $n_{2}$ values beyond $m=50-54$ are uncertain because when the sample is cooled, high $J$ lines become weak and often disappear from lower temperature spectra. For air broadening, there are no previous measured temperature dependence exponents available for this band. The temperature dependence exponents for air-broadening are larger than those for self-broadening, and the variation of $n_{1}$ vs. $m$ mirrors the $m$-variation seen in $n_{2}$. 
In Fig. 8(b) we have plotted previously measured values for the $30013 \leftarrow 00001(1.6-\mu \mathrm{m})$ band of ${ }^{12} \mathrm{C}^{16} \mathrm{O}_{2}$ [58]. The behavior of $n_{1}$ and $n_{2}$ vs. $m$ is similar to that obtained for the $20013 \longleftarrow 00001$ band shown in Fig. 8(a). A similar pattern was observed in calculated temperature dependence exponents for self-broadening reported by Predoi-Cross et al. [59] for the $30012 \longleftarrow 00001$ and the $30013 \leftarrow 00001$ bands of ${ }^{12} \mathrm{C}^{16} \mathrm{O}_{2}$ where the authors modeled their self-broadened half-width coefficients using semi-classical calculations based upon the Robert-Bonamy formalism [60]. The values in [61] for $n_{1}$ and in [59] for $n_{2}$ are not included in Fig. 8(b) for two reasons: (1) these $1.6 \mu \mathrm{m}$ bands were measured using a different [59] line mixing approach, and (2) the results were more scattered, especially for $n_{2}$, making it difficult to decipher. In Fig. 8(c) we have included the predicted temperature dependence exponents for $n_{1}$ and $n_{2}$ by Gamache and Lamouroux [57]. The variations in $n_{1}$ and $n_{2}$ with $m$ [57] show overall similar patterns observed in (a) and (b).

\subsection{Air-and self-shift coefficients and their temperature dependences}

Air- and self- pressure-shift coefficients, $\delta^{0}$ (air) and $\delta^{0}$ (self), and their temperature dependences $\delta^{\prime}$ (air) and $\delta^{\prime}$ (self) are measured for lines of the dominant $20013 \longleftarrow 00001$ band of ${ }^{12} \mathrm{C}^{16} \mathrm{O}_{2}$. Measurements were also obtained for a number of transitions in the $21113 \leftarrow 01101$ hot band of ${ }^{12} \mathrm{C}^{16} \mathrm{O}_{2}$. Similar to the Lorentz half-width coefficients, the pressure-shift coefficients were also measured for a number of the stronger transitions in a few other bands (see the Supplemental file).

The measured parameters for the $20013 \leftarrow 00001$ band are displayed in Fig. 9. The pressure-shift coefficients are plotted in the top panel (a). Values from the PS are compared to the measured values by Toth et al. $[\mathbf{1 2 , 1 3}]$ and values from the HITRAN2012 compilation. For $m(-20$ to +20$)$, the self-shift coefficients from PS are larger (more negative) compared to [12] while the air-shift coefficients are more consistent to those in [13]. The air-shift coefficients in HITRAN2012 are calculated values using experimental measurements modeled with empirical polynomial expressions and therefore follow a smooth curve with $m$. The HITRAN2012 compilation does not list self-shift coefficients. 


\section{Insert Fig. 9 here}

The self-shift coefficients from PS (solid red up triangles) are more strongly negative than those from Toth et al. [12] and follow a strange pattern, especially for $m$ in the -20 to +20 range. The reason for this is not obvious, but it could arise from strong correlation between pressure shifts and line mixing, resulting in some interplay in the observed pressure shifts and the large relaxation matrix element coefficients seen for $\mathrm{CO}_{2}-\mathrm{CO}_{2}$ mixing between the P-branch transitions (discussed later in the line mixing section). Normally, self-shift coefficients are measured more precisely than the air-shift coefficients due to the larger optical densities in pure sample spectra. In the PS, the self-shift coefficients do show more scatter and higher uncertainties than the air-shift coefficients (solid blue triangles).

The temperature dependences of the air- and self-shift coefficients, $\delta^{\prime}$ (air) and $\delta^{\prime}$ (self), are shown in Fig. 9(b). Similar to the temperature dependence exponents for air- and self-broadened Lorentz half-width coefficients, the patterns seen in the temperature dependences of pressureshift coefficients are also quite different. Panel (b) shows that $\delta^{\prime}$ (self) is more negative compared to $\delta^{\prime}$ (air) until $m=30-34$, beyond which $\delta^{\prime}$ (self) are more positive compared to $\delta^{\prime}$ (air). $\delta^{\prime}$ (self) shows the largest negative value of nearly $-0.000045 \mathrm{~cm}^{-1} \mathrm{~atm}^{-1} \mathrm{~K}^{-1}$ near the lowest $J$ and slowly becomes less negative until its value reaches zero near $m=30-34$ and increases slowly to a positive value close to $+0.00002 \mathrm{~cm}^{-1} \mathrm{~atm}^{-1} \mathrm{~K}^{-1}$ near $m=50$ and higher. Beyond $m=50$, their values are subject to higher uncertainty due to weakness of these lines at low temperatures.

$\delta^{\prime}$ (air), starts near $-0.00001 \mathrm{~cm}^{-1} \mathrm{~atm}^{-1} \mathrm{~K}^{-1}$ around $m=0$ and becomes slightly more negative in the $\mathrm{R}$ branch for $m=20-24$ and remain close to zero until $m=50$; while its value in the $\mathrm{P}$ branch remain close to zero from $m=10$ until $m=50 ; \delta^{\prime}$ (self) and $\delta^{\prime}$ (air) cross zero temperature dependence coefficient near $m=30$. This mirror behavior in $\delta^{\prime}$ (air) and $\delta^{\prime}$ (self) is also seen in Fig. 9(c) where plots for the $30013 \leftarrow 00001$ band of ${ }^{12} \mathrm{C}^{16} \mathrm{O}_{2}$ are shown [58], except the crossing occurs near $+0.000015 \mathrm{~cm}^{-1} \mathrm{~atm}^{-1} \mathrm{~K}^{-1}$ for $m=40-44$. The differences in magnitudes between $\delta^{\prime}$ (air) and $\delta^{\prime}$ (self) for the $20013 \leftarrow 00001$ band shown in panel (b) and the $30013 \leftarrow 00001$ band shown in panel (c) may be related to the differences between the magnitudes of the pressure-shift coefficients themselves (wavenumber dependence of pressure-shift coefficients). 


\subsection{Off-diagonal relaxation matrix element coefficients and their temperature dependences}

As higher pressure spectra were added to the global least squares fittings, the need to include line mixing became obvious. Line mixing using the off-diagonal relaxation matrix element coefficients $\left(W_{\mathrm{ij}}\right.$ in $\left.\mathrm{cm}^{-1} \mathrm{~atm}^{-1}\right)$ were included using the formalism of Lévy et al. [26]. In the analysis we included the mixing only between the nearest neighbor transitions. To make the fitting program work smoothly, initially, the relaxation matrix element coefficients were slowly activated for a few transition pairs in the $\mathrm{P}$ and the $\mathrm{R}$ branches, and for $\mathrm{CO}_{2}$-air and $\mathrm{CO}_{2}-\mathrm{CO}_{2}$ mixing starting from the center of the band (i.e., lowest $J$ ) and slowly adding higher- $J$ transitions. Statistical weights of $2 J^{\prime \prime}+1$ were assigned to each line with the appropriate $J^{\prime \prime}$.

In principle, if the dataset contains spectra at multiple temperatures, the temperature dependence exponents of the relaxation matrix element coefficients should also be determinable. Similar to building the relaxation matrix elements starting with zero values initially, the temperature dependence exponents for both $\mathrm{CO}_{2}-\mathrm{CO}_{2}$ and $\mathrm{CO}_{2}$-air states could also be setup in both $\mathrm{P}$ and $\mathrm{R}$ branches with initial default values (e.g., 0.75, a value similar to the temperature dependence exponents for the Lorentz half-width coefficients) and their values carefully adjusted until all residuals characteristic of line mixing disappear. In practice, fitting the temperature exponents of the line mixing has proved challenging. In our analysis of the 1.6- $\mu \mathrm{m} \mathrm{CO}_{2}$ band [58] as well as the $2 v_{3}$ band of $\mathrm{CH}_{4}$ [62], we have not been very successful in retrieving the temperature dependence exponents for all of the measured relaxation matrix element coefficients. In this study, we have measured the temperature dependence exponents for low- $J$ to mid- $J$ transitions for both $\mathrm{CO}_{2}-\mathrm{CO}_{2}$ and $\mathrm{CO}_{2}$-air mixing in the $\mathrm{P}$ and $\mathrm{R}$ branches (e.g., $\mathrm{P} 6 \& \mathrm{P} 8-\mathrm{P} 30$ \& $\mathrm{P} 32$ and R6 \& R8-R28 \& R30 and listed in Table 9). In determining these temperature dependence exponents, we have assumed the same power-law model (Eq. 4) applied to the temperature dependence exponents of the Lorentz half-width coefficients.

The off-diagonal relaxation matrix element coefficients for $\mathrm{CO}_{2}-\mathrm{CO}_{2}$ and $\mathrm{CO}_{2}$-air mixing listed in Table 9 have been measured in the PS for the maximum $J$ up to 50 in the P branch and up to $J=46$ in the $\mathrm{R}$ branch. All other $W_{i j}$ elements that could not be retrieved were fixed to a default value of $0.004-0.005 \mathrm{~cm}^{-1} \mathrm{~atm}^{-1}$ at $296 \mathrm{~K}$ (for the nearest neighbor transitions) up to P58 \& P60 
in the P branch and R48 \& R50 in the R branch (corresponding to $J$ for which Lorentz half-width coefficients were obtained).

\section{Table 9 comes here}

The measured values of $W_{i j}\left(\mathrm{~cm}^{-1}\right.$ atm $^{-1}$ at $\left.296 \mathrm{~K}\right)$ are plotted in Fig. 10(a) as a function of $m$. The measured $W_{i j}$ vary from 0.0047 to 0.047 for $\mathrm{CO}_{2}-\mathrm{CO}_{2}$, and between 0.0064 and 0.0022 for $\mathrm{CO}_{2^{-}}$ air mixing in the $\mathrm{P}$ branch. In the $\mathrm{R}$ branch, the range of $W_{i j}\left(\mathrm{~cm}^{-1} \mathrm{~atm}^{-1}\right.$ at $\left.296 \mathrm{~K}\right)$ varies between 0.0043 and 0.028 for $\mathrm{CO}_{2}-\mathrm{CO}_{2}$, and from 0.0025 to 0.023 for $\mathrm{CO}_{2}$-air. From Table 9 and Fig. 10(a) it is apparent that the value of the relaxation matrix element coefficients slowly increase from the center of the band and reach the maximum value around $m=20$ and then fall off slowly until the highest $J$ for which measurements are made. The $W_{i j}$ coefficients are not the same (but close) for the same $m$ in both the $\mathrm{P}$ and $\mathrm{R}$ branches. Generally the measured $W_{i j}$ for $\mathrm{CO}_{2}-\mathrm{CO}_{2}$ are larger compared to $\mathrm{CO}_{2}$-air, perhaps analogous to the corresponding self- and air-broadened Lorentz half-width coefficients as observed in our previous and recent studies of the $1.6 \mu \mathrm{m} \mathrm{CO}_{2}$ band [e.g., 58]. In Fig. 10(a) we have plotted the results from our earlier room temperature measurements for the $20013 \longleftarrow 00001$ band [16] for purpose of comparison with the present measurements. It is clear that the relaxation matrix element coefficients from PS for $\mathrm{CO}_{2}$-air (blue inverted triangles) are comparable to those measured in [16] (purple stars) in both the $\mathrm{P}$ and $\mathrm{R}$ branches. However, the line mixing coefficients for the $\mathrm{P}$ branch from PS for $\mathrm{CO}_{2}-\mathrm{CO}_{2}$ (solid black up triangles) are higher than those determined from room temperature measurements (solid green squares) and extend to a few more higher- $J$ transition pairs while those for the R branch are nearly the same in both the PS and in [16]. In the R branch, range for $\mathrm{CO}_{2}-\mathrm{CO}_{2}$ mixing extends only up to R34 \& R36 transition pairs in the PS, but it was measured to slightly larger $J$ range $(\mathrm{R} 36 \& 38)$ in [16]. The maximum value for $W_{i j}$ in the $\mathrm{R}$ branch is $\sim 0.03$ $\mathrm{cm}^{-1} \mathrm{~atm}^{-1}$. In the $\mathrm{P}$ branch, the range of $W_{i j}$ for $\mathrm{CO}_{2}-\mathrm{CO}_{2}$ extends to $\mathrm{P} 46 \& \mathrm{P} 48$ in the PS and the maximum $W_{i j}$ is $0.047 \mathrm{~cm}^{-1} \mathrm{~atm}^{-1}$ compared to the range $\mathrm{P} 42-\mathrm{P} 44$ and maximum $W_{i j}$ of $\sim 0.035$ $\mathrm{cm}^{-1} \mathrm{~atm}^{-1}$ in [16]. In the PS, the maximum value of $W_{i j}$ for $\mathrm{CO}_{2}-\mathrm{CO}_{2}$ is shifted to higher $J(\sim 36)$ compared to the room temperature study for $J(\sim 28)$. A small upward shift in the $\mathrm{P}$ branch $W_{i j}$ for the $\mathrm{CO}_{2}-\mathrm{CO}_{2}$ mixing starts near P20-P22 and reaches a maximum of $0.047 \mathrm{~cm}^{-1} \mathrm{~atm}^{-1}$ for P38 \& P40 transitions; this is nearly 55\% higher than the value in the $\mathrm{R}$ branch. We are not able to 
explain this peculiar behavior, except for the fact that the hot band $21113 \leftarrow 01101$ (centered near the P-branch side of $20013 \longleftarrow 00001$ band) is in Fermi resonance with the $40002 \longleftarrow 10002$ band. Since line mixing is strongly correlated with pressure shifts, this peculiar pattern of $W_{i j}$ in Fig. 10(a) could be responsible for the odd pattern for self-shift coefficients shown in Fig. 9(a). Temperature dependence exponents of the relaxation matrix element coefficients were obtained only for low- $J$ to mid- $J$ transitions; their values are listed in Table 9 (but not included in the plots). For all unmeasured transition pairs, their values were fixed to either 0.8 or 0.7 (see Table 9). To avoid complexity in the plots, the relaxation matrix element coefficients, $W_{i j}$, for the $30013 \leftarrow 00001$ band [58] are not included in Fig. 10(a).

Line mixing was also measured for a few transition pairs for both $\mathrm{CO}_{2}-\mathrm{CO}_{2}$ and $\mathrm{CO}_{2}$-air in the $\mathrm{Q}$ branch of the $21113 \leftarrow 01101$ band of ${ }^{12} \mathrm{C}^{16} \mathrm{O}_{2}$. The results are appended at the end of Table 9. Although it was required to include line mixing for the $\mathrm{Q}$ branch of this hot-band to obtain a better fit, those values should be considered only as approximate at best. Temperature dependence exponents for those relaxation matrix element coefficients were fixed to a default value of 0.7 . Statistical weights of $2 J^{\prime \prime}+1$ were applied while retrieving those relaxation matrix element coefficients.

Fig. 10 comes here (Line mixing and speed dependence)

\subsection{Speed dependence}

Speed dependence of the Lorentz half-width coefficients were measured for 112 transitions

mostly belonging to the $20013 \leftarrow 00001$ and the $21113 \leftarrow 01101$ bands of ${ }^{12} \mathrm{C}^{16} \mathrm{O}_{2}$. These correspond to $\sim 20 \%$ of the constrained lines for the strong and medium strength transitions (see Table 5). Measurement of this parameter requires measuring well into the wings of the Lorentz profile, so spectra with more carbon dioxide (stronger lines) and good signal-to-noise ratios were required. The measured speed dependence parameters for the $20013 \leftarrow 00001$ band are plotted as a function of $m$ in Fig. 10(b) and compared to values from previous room temperature measurements [16]. 
The quadratic speed dependence of the Lorentz half-width coefficients was measured using the following expression from Ref. [63] and given in Eq. (15) below, similar to our previous studies $[e . g ., \mathbf{3 7 , 3 8 , 5 8 , 6 2 ]}$.

$$
b_{L}^{0}(v)=b_{L}^{0}\left(v_{m}\right)\left\{1+S\left[\left(\frac{v}{v_{p}}\right)^{2}-c\right]\right\}
$$

In the above expression, $v$ is the speed of the molecular collision, $v_{\mathrm{m}}$ is the mean speed of the molecular collision, $v_{\mathrm{p}}$ is the most probable speed of the collision, $S$ is the speed dependence parameter (SD) reported in this study, and $\mathrm{c}$ is a constant whose value is taken to be 1.5 . When Eq. (15) is implemented into the Voigt expression and integrated over all velocities of collision, the resulting real $\left(K_{S}\right)$ and imaginary $\left(L_{S}\right)$ parts for the line shape are given by Eq. (16).

$$
\begin{aligned}
& K_{S}(x, y, S)=\frac{2}{\pi} \int_{-\infty}^{\infty} e^{-v^{2}} v \tan ^{-1}\left\{\left[\frac{v+x}{y\left(S\left\{v^{2}-c\right\}+1\right)}\right]^{2}\right\} d v \\
& L_{S}(x, y, S)=\frac{1}{\pi} \int_{-\infty}^{\infty} e^{-v^{2}} v \ln \left\{\left[\frac{v+x}{y\left(S\left\{v^{2}-c\right\}+1\right)}\right]^{2}+1\right\} d v
\end{aligned}
$$

Where,

$x=\sqrt{\ln 2}\left|v-v_{0}\right| / \alpha_{D}, y=\sqrt{\ln 2} b_{L}^{0} / \alpha_{D}$, and $\alpha_{D}$ is the Doppler half-width $\left(\mathrm{cm}^{-1}\right)$.

For transitions for which speed dependence was not measured, values were fixed to 0.08 for P52P84 and for R40-R84 (see the last two columns in the Supplemental file). The measured values of the speed dependence plotted in Fig. 10(b) are compared to values from our room-temperature analysis [16]. It is clear that the results obtained in the PS are about 8-10\% larger compared to the peak values for the R- and P-branch transitions [16]; the change is more pronounced for the $|m|$ values between 10 and 20. No obvious reason could be offered for this discrepancy except for the fact that the present study included a number of low-temperature spectra while the previous study involved only room-temperature data. We also assumed that the speed dependence parameter is independent of both the broadening gas $\left(\mathrm{CO}_{2}\right.$ and air in this study) and the temperature of the gas sample, hence a single value common to both $\mathrm{CO}_{2}-\mathrm{CO}_{2}$ and $\mathrm{CO}_{2}$-air 
broadening has been assumed. As stated earlier, Lisak et al. [56] recently published theoretical prediction for the temperature dependence of the quadratic speed dependence parameter.

Speed dependence parameters were also measured for a number of transitions of the P- and Rbranches of $21113 \leftarrow 01101$ band, but they are not included in Fig. 10(b). Some of the new line shape models and updated databases including software for line mixing in $\mathrm{CO}_{2}$ should be considered for improving the accuracy in future analysis of laboratory data requiring atmospheric remote retrievals $[57,64-68]$.

\subsection{Influence of line mixing in fitting laboratory spectra}

To illustrate the influence of line mixing in the retrieved parameters, we plot in Fig. 11(a) an airbroadened carbon dioxide spectrum and its fit residuals in (b) where line mixing was excluded in the final fit, and in (c) with line mixing included. The final fit shown was obtained from the multispectrum fit of all 27 spectra, and the graphs in panels (a), (b) and (c) are shown for one spectrum, as an example, taken from the multispectrum fit. The plots clearly demonstrate the point that except for the residuals under the strongest $20013 \leftarrow 00001$ band due to neglect of line mixing, the entire wavenumber interval (4700-4930.5 $\mathrm{cm}^{-1}$ ) has been fitted with no other detectable residuals (c). The plots also illustrate that line mixing was not required in any other bands under the present experimental conditions of the spectra fitted. The graphs in Fig. 11 compare remarkably well to Fig. 6 by Hartmann et al. [25] illustrating the influence of line mixing on atmospheric retrievals of $\mathrm{CO}_{2}$ from space. Theoretically, line mixing could be measurable for all the bands producing absorption in the fitted region. In experimental data, it would be necessary to include spectra with very large optical densities and carbon dioxide molecules, such as those obtained with cavity ring-down and photo-acoustic techniques, not only for the major carbon dioxide isotopologue but also for each of the rarer isotopologues producing absorption.

Insert Fig. 11 here (influence of line mixing) 


\section{Uncertainties in some measured parameters}

To estimate the total error budget in our measurements it would be useful to provide a few details illustrating the precision (excluding systematic errors) obtained. We present the best precision achieved in all of the line parameters by simultaneously fitting a number of spectra with careful choice of their experimental physical conditions for the gas samples.

\subsection{Line positions and intensities}

Uncertainties in zero pressure line positions are plotted in Fig. 12 as a function of $J^{\prime}$ for the strongest band in the measured region, $20013 \leftarrow 00001$ band of ${ }^{12} \mathrm{C}^{16} \mathrm{O}_{2}$. The theoretical expressions (Eqs. 1-3) used in the fittings result in the smooth $J$-dependence of these uncertainties that follow a simple function as displayed in Eq. (17).

$$
\begin{aligned}
\varepsilon_{\mathrm{v}}^{2}= & \varepsilon_{G^{\prime}}^{2}+2 q \rho_{G^{\prime} B^{\prime}} \varepsilon_{G^{\prime}} \varepsilon_{B^{\prime}}+q^{2}\left(\varepsilon_{B^{\prime}}^{2}-2 \rho_{G^{\prime} D^{\prime}} \varepsilon_{G^{\prime}} \varepsilon_{D^{\prime}}\right)+2 q^{3}\left(\rho_{G^{\prime} H^{\prime}} \varepsilon_{G^{\prime}} \varepsilon_{H^{\prime}}-\rho_{B^{\prime} D^{\prime}} \varepsilon_{B^{\prime}} \varepsilon_{D^{\prime}}\right) \\
& +q^{4}\left(\varepsilon_{D^{\prime}}^{2}+2 \rho_{B^{\prime} H^{\prime}} \varepsilon_{B^{\prime}} \varepsilon_{H^{\prime}}\right)-2 q^{5} \rho_{D^{\prime} H^{\prime}} \varepsilon_{D^{\prime}} \varepsilon_{H^{\prime}}+q^{6} \varepsilon_{H^{\prime}}^{2}
\end{aligned}
$$

Here $\varepsilon$ is the one standard deviation uncertainty in the parameter in the subscript, $q$ is $J(J+1)$ and $\rho$ is the correlation coefficient in the solution between the two parameters in the subscript. For the constrained interacting bands this expression requires several more terms involving the upper state constants for both interacting bands and the interaction coefficient. Both the values of $\varepsilon$ and $\rho$ are given in Table 6 for the constrained bands.

The uncertainties in positions for $0 \leq J \leq 40$ are between $0.6 \times 10^{-7}$ and $1.1 \times 10^{-6} \mathrm{~cm}^{-1}$, less than $1.1 \times 10^{-5} \mathrm{~cm}^{-1}$ for $J^{\prime}$ up to 60 , less than $1.1 \times 10^{-4} \mathrm{~cm}^{-1}$ for $J^{\prime}$ up to 80 . As always, the extrapolation of the uncertainties beyond the range of measured lines are only an estimate assuming that there are no local or non-local resonances or higher order ro-vibrational terms used in the equations.

\section{Insert Fig. 12 (Position uncertainty vs. $m$ )}

The uncertainties in the constrained non interacting intensities are computed as indicated in Eqs. (18-21). 
$\varepsilon_{S_{i}}^{2}=S_{i}^{2}\left\{\frac{\varepsilon_{S_{i}}^{2}}{S_{v}^{2}}+\frac{A_{1}}{F^{2}}+\frac{2}{F}\left[\frac{\varepsilon_{S_{i}} A_{2}}{S_{v}}+\frac{A_{3}}{F}\right]\right\}$

where, $F$ is the Herman-Wallis factor as defined in Eq. (3), and

$$
\begin{aligned}
& A_{1}=m^{2} \varepsilon_{a_{1}}^{2}+m^{4} \varepsilon_{a_{2}}^{2}+m^{6} \varepsilon_{a_{3}}^{2}+\left[J^{\prime}\left(J^{\prime}+1\right)\right]^{2} \varepsilon_{a_{4}}^{2} \\
& A_{2}=\rho_{S_{i} a_{1}} m \varepsilon_{a_{1}}+\rho_{S_{i} a_{2}} m^{2} \varepsilon_{a_{2}}+\rho_{S_{i} a_{3}} m^{3} \varepsilon_{a_{3}}+\rho_{S_{i} a_{4}} J^{\prime}\left(J^{\prime}+1\right) \varepsilon_{a_{4}} \\
& A_{3}=\rho_{a_{1} a_{2}} m^{3} \varepsilon_{a_{1}} \varepsilon_{a_{2}}+\rho_{a_{1} a_{3}} m^{4} \varepsilon_{a_{1}} \varepsilon_{a_{3}}+\rho_{a_{1} a_{4}} m J^{\prime}\left(J^{\prime}+1\right) \varepsilon_{a_{1}} \varepsilon_{a_{4}} \\
& +\rho_{a_{2} a_{3}} m^{5} \varepsilon_{a_{2}} \varepsilon_{a_{3}}+\rho_{a_{2} a_{4}} m^{2} J^{\prime}\left(J^{\prime}+1\right) \varepsilon_{a_{2}} \varepsilon_{a_{4}}+\rho_{a_{3} a_{4}} m^{3} J^{\prime}\left(J^{\prime}+1\right) \varepsilon_{a_{3}} \varepsilon_{a_{4}}
\end{aligned}
$$

The definitions of uncertainties, $\varepsilon$ and $\rho$, appearing in the various terms in Eqs. (18)-(21) are the same as those in Eq. (17). Expressions for the interacting bands will require many additional terms for the upper states of both the bands involved in the interaction and the interaction coefficient than provided in the above Equations. The values of $\varepsilon$ and $\rho$ for the constrained interacting bands are listed in Table 6.

The percent uncertainties in the fitted intensities (ratio of intensity uncertainty to intensity $\times 100$ ) are plotted as a function of $m$ in Fig. 13 for the $20013 \longleftarrow 00001$ band of ${ }^{12} \mathrm{C}^{16} \mathrm{O}_{2}$. The percent uncertainty in intensity varies between $0.005 \%$ and $0.01 \%$ for $|m| 0 \leq 10 \leq 50$ and to $\sim 0.1 \%$ for $|m|=100$ (notice that there is a minimum percent intensity of $\sim 0.003 \%$ near P24). Such high precision in positions (Fig. 12) and intensities (Fig. 13) was achieved by using the constraints as discussed earlier. The absolute error in measured individual line intensities is limited by the uncertainty due to possible dimer formation that may occur in the sample at cold temperatures or if there is any Collision Induced Absorption (CIA) in addition to systematic errors arising from knowledge of absorption path lengths, pressure and temperature readings and model deficiencies.

Insert Fig. 13 (Percent intensity uncertainty vs. $m$ ) 


\subsection{Lorentz half-width coefficients vs. sample temperature}

In order to simulate the spectrum of a molecule or its band(s) at a specific temperature, the uncertainties associated with the spectral line parameters at that temperature are even more important than the uncertainties in their values themselves. If we measure only the half-width coefficients at $\mathrm{T}=296 \mathrm{~K}$ and apply its temperature dependence from another source (such as the calculated values available in the HITRAN compilation), it is assumed that the half-width coefficients near $296 \mathrm{~K}$ are the best determined values, and the uncertainty in the knowledge of temperature dependence exponent $(n)$ increases the uncertainty in half-width coefficients as the sample temperature moves father from $296 \mathrm{~K}$ (at which our measurements are reported). This is not always the case as we illustrate in the following discussion supported by the present measurements.

In Fig. 14, we have plotted the uncertainties (internal one sigma values) in the measured Lorentz half-width coefficients $\left(\mathrm{cm}^{-1} \mathrm{~atm}^{-1}\right)$ vs. gas sample temperatures (in K) for a set of self- and airbroadened transitions for the $20013 \leftarrow 00001$ band of ${ }^{12} \mathrm{C}^{16} \mathrm{O}_{2}$. We have shown the variations of half-width uncertainties vs. gas temperature for P4, P18, and R42 transitions to represent low-J, medium- $J$ and high- $J$ transitions. For each graph, the uncertainties without considering the correlations are also plotted to illustrate the significance of incorporating correlations in calculating the uncertainties. For the low- to medium $J$ transitions, the minimum uncertainty tends to be towards the low temperature (200-230 K) range, whereas for the higher $J$ transitions, the minimum uncertainty gets shifted to higher temperature (typically between 270 and $290 \mathrm{~K}$ ). There are no experimental data above room temperature from this study to see the pattern in uncertainty (the curves drawn above room temperature and below $170 \mathrm{~K}$ are extrapolated).

For high- $J$ transitions, the intensities of the transitions become weak at low temperatures and the fit does not determine the half-width coefficients well. Therefore, the temperature region where the half-width coefficients are best determined narrows to only the higher temperatures of the fitted region. It is obvious that the trend toward lower uncertainties at higher temperature for higher $J$ transitions (e.g., R42 self- at the top curve) is akin to the trend towards lower uncertainties at lower temperature for the low- $J$ and mid- $J$ values (e.g., P4 self-). 


\section{Insert Fig. 14 (uncertainty in Lorentz width vs. temp)}

In Fig. 15, graphs showing the relative uncertainty (ratios of half-width uncertainty to half-width coefficients) are plotted vs. gas sample temperature (in K) for the same set of transitions shown in Fig. 14. The relative uncertainties with and without considering the correlations among fitted parameters are shown by dashed curves. As in Fig. 14, the percent uncertainties remain small for low to mid-J transitions. Similar in Fig. 14, to minimize congestion, only few transitions are labeled.

The uncertainty in the Lorentz halfwidth coefficient at temperature $T$ is given Eq. (22).

$\varepsilon_{b_{L}^{0}(T)}^{2}=b_{L}^{0}(T)^{2}\left\{\frac{\varepsilon_{b_{L}^{0}\left(T_{0}\right)}^{2}}{b_{L}^{0}\left(T_{0}\right)^{2}}+\left[\ln \left(\frac{T_{0}}{T}\right)\right]^{2} \varepsilon_{n}^{2}+2 \rho_{b_{L}^{0}\left(T_{0}\right), n} \frac{\ln \left(\frac{T_{0}}{T}\right) \varepsilon_{b_{L}^{0}\left(T_{0}\right)} \varepsilon_{n}}{b_{L}^{0}\left(T_{0}\right)}\right\}$

Here $\varepsilon$ is the one standard deviation uncertainty in the parameter in the subscript, and $\rho$ is the correlation coefficient in the solution between the two parameters in the subscript.

\section{Insert Fig. 15 (Relative uncertainty in Lorentz width vs. temp)}

\subsection{Uncertainties in pressure shift coefficients vs. sample temperature}

Uncertainties in air- and self- pressure-shift coefficients vs. gas sample temperature (in K) for the P18, P42, R4 and R18 transitions are plotted in Fig. 16 for the ${ }^{12} \mathrm{C}^{16} \mathrm{O}_{2} 20013 \leftarrow 00001$ band. Similar to Fig. 14, the uncertainties in the pressure-shift coefficients vs. temperature also tend to be smallest near the middle of the temperature range of the fitted spectra. Uncertainties in airshift coefficients are smaller than those for self-shift coefficients for all transitions plotted. In the present study, such large differences in pressure-shift uncertainty between air- and self-shift coefficients could be due to the much larger relaxation matrix element coefficients for $\mathrm{CO}_{2}-\mathrm{CO}_{2}$ mixing obtained from present study (Fig. 10(a)), and the large correlations with self-shift coefficients. Graphs with correlations included in the fit are not shown in this figure. For low- $J$ and medium- $J$ transitions, the uncertainty in air-shift coefficients is small for all temperatures in the fitted temperature range. Eq. (23) is used for determining the pressure-shift uncertainty. 


$$
\varepsilon_{\delta}^{2}=\varepsilon_{\delta_{0}}^{2}+\left(T-T_{0}\right)^{2} \varepsilon_{\delta^{\prime}}^{2}+2 \rho_{\delta_{0} \delta^{\prime}}\left(T-T_{0}\right) \varepsilon_{\delta_{0}} \varepsilon_{\delta^{\prime}}
$$

Similar to Eqs. (17)-(22), here $\varepsilon$ is the one standard deviation uncertainty in the parameter indicated by the subscript, and $\rho$ is the correlation coefficient in the solution between the two parameters in the subscript.

Insert Fig. 16 (uncertainty in pressure shift vs. gas temperature)

\subsection{Uncertainties in measured relaxation matrix coefficients vs. sample temperature}

Uncertainty in the measured off-diagonal relaxation matrix element coefficients $\left(\mathrm{cm}^{-1} \mathrm{~atm}^{-1}\right)$ are plotted vs. gas sample temperature (in K) in Fig. 17. Plots are shown for two transition pairs, P12 \& P14 and R12 \& R14 (for $\mathrm{CO}_{2}-\mathrm{CO}_{2}$ and $\mathrm{CO}_{2}$-air) as marked in the panel. Uncertainties without and with correlations are computed as a function of sample temperature to illustrate their importance in reducing the measured uncertainty which are clearly observed for the two cases of $\mathrm{CO}_{2}-\mathrm{CO}_{2}$ mixing. As seen in Fig. 17, the uncertainty is much larger for $\mathrm{CO}_{2}-\mathrm{CO}_{2}$ than for $\mathrm{CO}_{2}$-air. This behavior could be related to the peculiar relaxation matrix element coefficients mentioned earlier and shown in Fig. 10(a) and listed in Table 9. Compared to $\mathrm{CO}_{2}-\mathrm{CO}_{2}$, the uncertainty for $\mathrm{CO}_{2}$-air mixing is smaller by a factor of three or more.

Eq. (22) used for uncertainty in Lorentz half-width coefficients could be applied for the line mixing uncertainty (except replacing half-width coefficient $b_{L}^{0}$ with the line mixing coefficient $\left.W_{i j}\right)$.

Insert Fig. 17 (Uncertainty in relaxation matrix coefficients vs. gas sample temperature)

Plots similar to Figs. 14-17 could be made for all measured line parameters obtained from this study. Representative cases only are discussed here to show that for the accurate simulation of spectra it is not the uncertainties in the Lorentz half-width or pressure shift at $296 \mathrm{~K}$ of those 
transitions that are important, but the uncertainties in those parameters at the temperature at which the spectrum is to be calculated.

\section{Estimated total error budget}

With several spectral line parameters investigated in the present study, it is important to provide the absolute errors we estimate in the various measured quantities. Where possible, each measured parameter was listed with its associated uncertainty (in various Tables). These values correspond to the formal one-sigma precisions resulting from the simultaneous fitting of several spectra. To judge the absolute errors in the retrieved parameters, the errors in the experimental conditions of the spectra and reference standards used in the calibration of wavenumber scales must be evaluated. These errors include uncertainties in the knowledge of absorption cell path lengths, knowledge of gas pressures and temperatures, calibration errors in their monitoring systems, errors due to line shape model deficiencies, weak absorption features whose parameters could not be adjusted in the fits and hence fixed to some theoretical or default values in the least squares solution. Understanding, evaluating and fixing those errors is not trivial, and every effort was made to minimize these errors sources. Simultaneous fitting of several spectra recorded at different gas conditions and using various absorption path cells, including non-Voigt line shapes and the best-fit obtained (Figs. 1 and 2) with no significant fit residuals clearly indicate that most such uncertainties are small. The absolute one sigma standard deviations in our measured parameters are estimated conservatively to be $0.0001 \mathrm{~cm}^{-1}$ for positions, $\leq 1 \%$ for line intensities, $<2-3 \%$ for the Lorentz half-width coefficients (for the strong, unblended lines), 5-10\% in pressure-shift coefficients and in the temperature dependences of width and shift coefficients. The absolute errors in the off-diagonal relaxation matrix elements and their temperature dependence exponents, and the speed dependence parameters are estimated to be in the 5-20\% (depending on individual lines, their rotational quantum numbers, $\mathrm{S} / \mathrm{N}$ etc.).

To compute the absolute accuracies in our measured line intensities we have estimated the various contributions due to systematic error sources: (a) $0.3-0.4 \%$ in the knowledge of path lengths, (b) 0.1-0.2\% in pressure readings, (c) 0.1-0.2\% in temperature readings, and (d) 0.1$0.2 \%$ background polynomial fits, field of view corrections, residual phase errors, zero level determinations, and some undetermined contribution due to model deficiencies in our analysis. 
The global standard deviation of weighted observed minus calculated fit residuals are only $0.045 \%$, thus eliminating almost all random errors to within the noise levels of the fitted spectra.

After we completed our analysis and in the process of documenting the results, new calculations of line intensities became available. In Fig. 18 we provide comparisons of our measured line intensities to the only other extensive experimental measurements of Toth et al. [11], calculated line intensities from HITRAN2012 $[\mathbf{3 6 , 4 5}]$ and the recent $a b$ initio calculations by Zak et al. [50]. In the top panel we have shown the four sets of line intensities (cm/molecule at $296 \mathrm{~K}$ ) plotted vs. $m$. In the middle panel (b) we show the position differences between present values and those from Zak et al. The mean and standard deviation of the position differences, (PS-Zak et al.) for P68-R68 is $0.00022(4) \mathrm{cm}^{-1}$. In the bottom panel (c) the ratios of present line intensities to HITRAN database [36,45], Toth et al. [11] and the ab initio values [50] are shown. The following mean ratios with standard deviations are obtained: PS/HITRAN2012=0.993(5); PS/HITRAN2008=0.988(8); PS/Toth et al. [11]=0.992(5) and PS/ab initio $=0.994(2)$. Similar observations were made in our study of the 1.6 micron $\mathrm{CO}_{2}$ band [58].

Insert Fig. 18 here [Comparisons of Intensities with ab initio calculations]

Judging the various plots in Fig. 18(c), very good agreement is seen between present measurements and with those in [45] and [50] for the entire P84-R84 range. Similar agreement is also seen between PS and Toth et al., where the range in $J$ is limited to P58-R58. A small curvature in the intensity ratios between this study and the ab initio calculations is clear which could be related to the differences in the models used in the two methods.

Calculations of new line positions and intensities for $\mathrm{CO}_{2}$ including the 2- $\mu \mathrm{m}$ region have been reported recently [48-50]. For a comprehensive understanding of the changes in the $\mathrm{CO}_{2}$ line positions and intensities (including the $20013 \leftarrow 00001$ band of ${ }^{12} \mathrm{C}^{16} \mathrm{O}_{2}$ ) listed in HITRAN databases $[\mathbf{3 6 , 4 4 , 4 5}]$, and to compare our results with the most recent calculations [46-50], we include two figures [Figs. 19 and 20]. In Fig. 19(a) comparison of line position differences between HITRAN2012 [45] and CDSD-296 [48] vs. $m$ and in 19(b) percent intensity differences between HITRAN2012 and CDSD-296 vs. $m$ are shown. In both (a) and (b) the values corresponding to higher $m$ ( $\sim 60$ and beyond shown by solid red circles) are predicted values 
from various sources (see Section 4). As seen in Fig. 19, the position differences between HITRAN2012 and CDSD-296 lie between +0.000033 and $+0.000184 \mathrm{~cm}^{-1}$ for P58-R62, and beyond that the differences range from -0.00012 to $+0.00283 \mathrm{~cm}^{-1}$. For the same range in $J$, the $\%$ intensity differences between HITRAN2012 and CDSD-296 are small and vary approximately from -0.9 to +1.1 and from -0.3 to -1.0 , respectively.

\section{Insert Fig. 19 here (Comparisons between HITRAN2012 vs. CDSD-296)}

In Fig. 20, the ratios of line intensities between PS and $[\mathbf{1 1 , 3 6 , 4 5 , 4 8 - 5 0 ]}$ vs. $m$ are plotted. We find that line intensities from present study agree well with all the comparisons plotted in (a). The mean and standard deviations of the ratios of line intensities between this study with Toth et al. $[11]=0.992(5) ;$ HITRAN2012 $=0.993(5) ;$ HITRAN2008 $=0.988(7)$, Zak et al. $=0.994(3)$; CDSD-296 $=0.990(3)$; and Huang $=0.981(5)$. While the comparisons with Toth et al. are limited to their measured values for P58-R62, all other comparisons are for P84-R84. In panel (b), the following mean ratios for the plots shown are obtained: Zak et al./CDSD-296 $=0.996(5)$; Zak et al./Huang $=0.997(2)$, and CDSD-296/Huang $=0.991(6)$. It is interesting to see that the curvature observed in the intensity ratios between PS/Zak et al. and between PS/CDSD-296 seen in panel (a) is also seen in the plots showing the intensity ratios among other ab initio $[49,50]$ and calculated [48] intensities, though to a lesser degree.

Line mixing (off-diagonal relaxation matrix formalism) included in the PS could influence line intensities, although its magnitude is not quantified at this time. The very good agreement (0.994 \pm 0.003$)$ in line intensities between PS and the ab initio calculated intensities [50] is very encouraging.

In addition to plotting the intensity ratios discussed above, we have shown several other comparisons in panels (c) - (f). In (c) line position differences PS-Zak et al. and PS-CDSD-296 are shown, (d) Ratios of Lorentz air- and self-broadened half-width coefficients between PS and CDSD-296, (e) temperature dependence exponents of Lorentz air-broadened half-width coefficients from PS and CDSD-296, and (f) air- pressure-shift coefficients from PS and CDSD296. In addition to the good agreement seen between present measurements and CDSD-296 for 
line positions and intensities as seen in panels (a) and (c) for P64-R64, it is very encouraging to see also the good agreements in the self- and air-broadened half-width coefficients, temperature dependence exponents for air-broadened half-width coefficients and air- pressure-shift coefficients obtained from PS and the CDSD-296.

\section{Insert Fig. 20 here (Comparisons of several parameters)}

Comparisons of line positions and intensities between the present results and the CDSD-296 databank [48], Huang [49] and Zak et al. [50] and line shape parameters with CDSD-296 are compared and plotted.

\section{Conclusions}

The principal objective of this investigation was to support accurate $X_{\mathrm{CO} 2}$ remote retrievals for the OCO-2 mission. As for the $1.6 \mu \mathrm{m}$ region [58], the present results will be used to generate the absorption coefficients for the OCO-2 retrieval algorithm; these results can also be applied to model spectra for other instruments (such as GOSAT) measuring the 2.06 and $1.6 \mu \mathrm{m} \mathrm{CO}_{2}$ bands.

Recent multiple studies have shown that accurate simulations of remote retrievals for well-mixed atmospheric gases (e.g., $\mathrm{CO}_{2}, \mathrm{CH}_{4}$ ) require spectroscopic characterization of molecular line parameters beyond the use of traditional Voigt line shapes to minimize measurement uncertainties without systematic residuals. In the present work we have reduced the fit residuals in our laboratory absorption spectra of pure $\mathrm{CO}_{2}$ and mixtures of $\mathrm{CO}_{2}$ in air by including speed dependence and line mixing via off-diagonal relaxation matrix element coefficients. We have further improved the analysis by reducing random errors through simultaneously fitting a large number of experimental spectra (recorded at high resolution and high S/N acquired at various opacities, absorption path lengths, gas amounts and sample temperatures). Important precautionary measures were followed with great care in collecting the laboratory data by carefully monitoring the physical conditions of the data while data were being recorded. New fitting methodologies were implemented for determining the ro-vibrational and band intensity 
parameters by constraining the individual line positions and intensities to the known theoretical quantum mechanical expressions. Spectroscopic parameters were determined for several bands that reproduce detectable absorptions in the fitted region by including many weaker features and interfering bands to increase the accuracy of the fits. The high correlations between line intensities and Lorentz half-width coefficients were reduced by appropriate choice of the experimental conditions of the spectra.

The goal of the OCO-2 mission is to retrieve $X_{C O 2}$ with accuracy of 1 part in $400(0.25 \%)$. Spectroscopy is only one part of the OCO-2 error budget [69]. Therefore, in order to achieve the OCO-2 accuracy goal, the spectroscopic parameters must be known to even higher accuracy. Through the results presented in this work, we have demonstrated that with careful planning and implementing a state-of-the art analysis technique, it was possible to characterize $\mathrm{CO}_{2}$ spectral line parameters (positions, intensities and line shape parameters) with precision close to $\sim 0.3 \%$.

There is still room for improvement in future analyses. For example, off-diagonal relaxation matrix elements for only the neighboring pairs of transitions have been considered, but it is possible that line mixing between non-neighboring transitions could occur. Furthermore, speed dependence associated with only the Lorentz half-width coefficients was included in this study. However, new studies involving different line shape models are appearing in the literature, such as more sophisticated line shapes including speed dependence of pressure-shift coefficients and temperature dependence of speed dependence are being evaluated. Also, assumptions were made in this work for retrieving the temperature dependences of Lorentz half-width (power-law) and pressure-shift coefficients (linear model); the speed dependence was assumed to be independent of the broadening gas and independent of gas temperatures Perhaps a standard line shape model including line mixing, speed dependence and other more subtle effects such as Dicke narrowing and collision-induced absorption could be required to establish inter-laboratory comparisons and standardization of spectra databases. The situation is complicated by the fact that a single choice of line shape/line mixing formalism is unlikely to produce optimal results under all pressuretemperature combinations of all broadening gases.

We emphasize that the parameterization presented in this study is intended for specific applications for $\mathrm{CO}_{2}$ retrievals for earth's atmospheric remote sensing. The present 
measurements of the temperature dependences for the air- and self-broadened Lorentz widths, pressure shifts and the relaxation matrix element coefficients along with speed dependence result in best precision for the $2.06 \mu \mathrm{m}$ region for the pressure and temperature conditions applicable to earth's atmosphere. We conclude that, while simulating the spectrum of $\mathrm{CO}_{2}$, the full accuracy will be achieved only by using the complete, consistent set of parameters; the full accuracy will be reduced if some of the parameters from the present study are applied to another line shape/line mixing model or mixed with parameters obtained from other studies.

\section{Acknowledgments}

Part of the material related to Kitt Peak measurements applied in this investigation was based upon work supported by the National Science Foundation under Grant \# ATM-0338475 to the College of William and Mary. The recent study involving the low-temperature measurements was supported by a Research Grant to the College of William and Mary for the OCO-2 mission through JPL. The research at the Jet Propulsion Laboratory (JPL), California Institute of Technology, Connecticut College and NASA Langley Research Center was performed under contracts and cooperative agreements with the National Aeronautics and Space Administration.

One of the authors, RRG, was supported by the National Science Foundation through Grant \# AGS-1156862. DCB and VMD acknowledge the help provided by Emily M. Nugent, a former student of the College of William and Mary. 


\section{References}

1. D. Crisp, R.M. Atlas, F.-M. Breon, L.R. Brown, J.P. Burrows, P. Ciais, et al. The Orbiting carbon Observatory (OCO) mission. Adv. Space. Res. 34 (2004) 700-709.

2. T. Yokota, Y. Yoshida, N. Eguchi, Y. Ota, T. Tanaka, H. Watanabe, et al. Global concentrations of $\mathrm{CO}_{2}$ and $\mathrm{CH}_{4}$ retrieved from GOSAT: first preliminary results. SOLA Sci. Online: Lett. Atmos. 5 (2009) 160-163. doi:10.2151/Sola.2009-041

3. H. Boesch, D. Baker, B. Connor, D. Crisp, C.E. Miller. Global characterization of $\mathrm{CO}_{2}$ column retrievals from shortwave-infrared satellite observation of the Orbiting Carbon Observatory-2 mission. Remote. Sens. 3 (2011) 270-304.

4. C.E. Miller, D. Crisp, P.L. DeCola, S.C. Olsen, J.T. Randerson, A.M. Michalak, et al. Precision requirements for space-based $\mathrm{X}_{\mathrm{CO} 2}$ data. J. Geophys. Res. 112 (2007) D10314. doi:10.1029/2006JD007659.

5. D. Crisp, C.E. Miller, P.L. DeCola. NASA Orbiting Carbon Observatory: measuring the column averaged carbon dioxide mole fraction from space. J. Appl. Rem. Sens. 2 (2008) 023508. http://dx.doi.org/10.1117/1.2898457.

6. D.R. Thompson, D.C. Benner, L.R. Brown, D. Crisp, V.M. Devi, J. Yibo, et al. Atmospheric validation of high accuracy $\mathrm{CO}_{2}$ absorption coefficients for the OCO-2 mission. J. Quant. Spectrosc. Radiat. Transfer 113 (2012) 2265-2276.

7. D. Crisp, B.M. Fischer, C. O’Dell, C. Frankenberg, R. Basilio, H. Bösch, et al. The ACOS $\mathrm{CO}_{2}$ retrieval algorithm-Part II. Global $\mathrm{X}_{\mathrm{CO} 2}$ data characterization. Atmos. Meas. Tech. 5 (2012) 687-707.

8. C.W. O’Dell, B. Connor, H. Bösch, D. O’Brien, C. Frankenberg, R. Castano, et al. The ACOS $\mathrm{CO}_{2}$ retrieval algorithm Part I: Description and validation against synthetic observations. Atmos. Meas. Tech. Discuss. 5 (2012) 99-121. doi:10.5194/amt-5-99-2012.

9. R.A. Washenfelder, G.C. Toon, J.-F Blavier, Z. Yang, N.T. Allen, P.O. Wennberg, et al. Carbon dioxide column abundances at the Wisconsin Tall Tower site. J. Geophys. Res.111 (2006) D22305. doi:10.1029/2006JD007154 
10. D. Wunch, G.C. Toon, J.-F. Blavier, R.A. Washenfelder, J. Nothold, B. Connor, et al. The Total Carbon Column Observing Network. Philo. Trans. R. Soc. A 369 (2011) 2087-2112.

11. R.A. Toth, L.R. Brown, C.E. Miller, V.M. Devi, D.C. Benner. Line strengths of ${ }^{12} \mathrm{C}^{16} \mathrm{O}_{2}$ : 4550-7000 $\mathrm{cm}^{-1}$. J. Mol. Spectrosc. 239 (2006) 221-242.

12. R.A. Toth, L.R. Brown, C.E. Miller, V.M. Devi, D.C. Benner. Self-broadened widths and shifts of ${ }^{12} \mathrm{C}^{16} \mathrm{O}_{2}$ : 4750-7000 cm ${ }^{-1}$. J. Mol. Spectrosc. 239 (2006) 243-271.

13. R.A. Toth, C.E. Miller, V.M. Devi, D.C. Benner, L.R. Brown. Air-broadened halfwidth and pressure shift coefficients of ${ }^{12} \mathrm{C}^{16} \mathrm{O}_{2}$ bands: 4750-7000 $\mathrm{cm}^{-1}$. J. Mol. Spectrosc. 246 (2007) 133157.

14. R.A. Toth, C.E. Miller, L.R. Brown, V.M. Devi, D.C. Benner. Line Positions and strengths of ${ }^{16} \mathrm{O}^{12} \mathrm{C}^{18} \mathrm{O},{ }^{18} \mathrm{O}^{12} \mathrm{C}^{18} \mathrm{O}$ and ${ }^{17} \mathrm{O}^{12} \mathrm{C}^{18} \mathrm{O}$ between 2200 and $7000 \mathrm{~cm}^{-1}$. J. Mol. Spectrosc. 243 (2007) 43-61.

15. R.A. Toth, L.R. Brown, C.E. Miller, V.M. Devi, D.C. Benner. Spectroscopic database of $\mathrm{CO}_{2}$ line parameters: 4300-7000 cm c $^{-1}$ J. Quant. Spectrosc. Radiat. Transfer 109 (2008) 906-921.

16. D.C. Benner, V.M. Devi, E. Nugent, L.R. Brown, C.E. Miller, R.A, Toth, K. Sung. The 4850 $\mathrm{cm}^{-1}$ spectral region of $\mathrm{CO}_{2}$ : Constrained multispectrum nonlinear least squares fitting including line mixing, speed dependent line profiles and Fermi resonance. Paper presented at the $64^{\text {th }}$ International Symposium on Molecular Spectroscopy, Ohio State University. June 22-26 ${ }^{\text {th }}, 2009$. (\# RI12, pp. 266).

17. C.B. Suarez, F.P.J. Valero. Temperature dependence of self-broadened halfwidths of $\mathrm{CO}_{2}$. J. Quant. Spectrosc. Radiat. Transfer 43 (1990) 327-334.

18. L. Régalia-Jarlot, V. Zénninari, B. Parvitte, A. Grossel, X. Thomas, P. Heyden von der, et al. A complete study of the line intensities of four bands of $\mathrm{CO}_{2}$ around 1.6 and $2.0 \mu \mathrm{m}$ : A comparison between Fourier transform and diode laser measurements. J. Quant. Spectrosc. Radiat. Transfer 101 (2006) 325-338. 
19. L. Joly, F. Gibert, B. Grouiez, A. Grossel, B. Parvitte, G. Durry, V. Zénninari. A complete study of $\mathrm{CO}_{2}$ line parameters around $4845 \mathrm{~cm}^{-1}$ for lidar applications. J. Quant. Spectrosc. Radiat. Transfer 109 (2008) 426-434.

20. L. Joly, F. Marnas, F.Gibert, D. Bruneau, B. Grouiez, P.H. Flamant, G. Durry, et al. Laser diode absorption spectroscopy for accurate $\mathrm{CO}_{2}$ line parameters at $2 \mu \mathrm{m}$ : Consequences for space-based DIAL measurements and potential biases. Appl. Opt. 48 (2009) 5475-5483.

21. J.S. Li, G. Durry, J. Cousin, L. Joly, B. Parvitte, P.H. Flamant, et al. Tunable diode laser measurement of pressure-induced shift coefficients of $\mathrm{CO}_{2}$ around $2.05 \mu \mathrm{m}$ for lidar applications. J. Quant. Spectrosc. Radiat. Transfer 112 (2011) 1411-1419.

22. J.S. Li, G. Durry, J. Cousin, L. Joly, B. Parvitte, V. Zénninari. Self-induced pressure shift and temperature dependence measurements of $\mathrm{CO}_{2}$ at $2.05 \mu \mathrm{m}$ with a tunable diode laser spectrometer. Spectrochim. Acta A 85 (2012) 74-78.

23. L.E. Christensen, G.D. Spiers, R.T. Menzies, J.C. Jacob. Tunable laser spectroscopy of $\mathrm{CO}_{2}$ near $2.05 \mu \mathrm{m}$ : Atmospheric retrieval biases due to neglecting line-mixing. J. Quant. Spectrosc. Radiat. Transfer 113 (2012) 739-748.

24. G. Casa, R. Wehr, A. Castrillo, E. Fasci, L. Gianfrani. The line shape problem in the nearinfrared spectrum of self-colliding $\mathrm{CO}_{2}$ molecules: Experimental investigation and test of semiclassical models. J. Chem. Phys. 130 (2009) 184306.

25. J.-M. Hartmann, H. Tran, G.C. Toon. Influence of line mixing on the retrievals of atmospheric $\mathrm{CO}_{2}$ from spectra in the 1.6 and $2.1 \mu \mathrm{m}$ regions. Atmos. Chem. Phys. 9 (2009) 7303-7312.

26. A. Levy, N. Lacome, C. Chackerian Jr. Collisional line mixing In: Rao K Narahari, Weber Alfons, editors. Spectroscopy of the earth's atmosphere and interstellar medium. San Diego, CA. Academic Press, Inc. 1992 (Chapter 4).

27. D.A. Long, C.-W. Truong, J.T. Hodges, C.E. Miller. Absolute ${ }^{12} \mathrm{C}^{16} \mathrm{O}_{2}$ transition frequencies at the kHz-level from 1.6 to $7.8 \mu \mathrm{m}$. J. Quant. Spectrosc. Radiat. Transfer 130 (2013) 112-115. 
28. D. Gatti, N. Coluccelli, A. Gambetta, A. Di Lieto, M. Tonelli, G. Galzerano, et al. Absolute frequency spectroscopy of $\mathrm{CO}_{2}$ lines around $2.09 \mu \mathrm{m}$ by combined use of an Er:fiber comb and a Ho:YLF amplifier. Opt. Lett. 36 (2011) 3921-3923.

29. D.C. Benner, C.P. Rinsland, V.M. Devi, M.A.H. Smith, D. Atkins. A multispectrum nonlinear least-squares fitting technique. J. Quant. Spectrosc. Radiat. Transfer 53 (1995) 705721.

30. K.L. Letchworth, D.C. Benner. Rapid and accurate calculation of the Voigt function. J. Quant. Spectrosc. Radiat. Transfer 107 (2007) 173-192.

31. K. Sung, A.W. Mantz, M.A.H. Smith, L.R. Brown, T.J. Crawford, V.M. Devi, et al. Cryogenic absorption cells operating inside a Bruker IFS-125HR: First results for ${ }^{13} \mathrm{CH}_{4}$ at $7 \mu \mathrm{m}$. J. Mol. Spectrosc. 262 (2010) 122-134.

32. A.W. Mantz, K. Sung, T.J. Crawford, L.R. Brown, M.A.H. Smith, V.M. Devi, et al. A cryogenic Herriott cell vacuum coupled to a FT-IR JPL Bruker IFS-125 HR. J. Mol. Spectrosc. 304 (2014) 12-24.

33. C.R. Pollock, P.R. Petersen, D.A. Jennings, J.S. Wells, A.G. Maki. Absolute frequency measurements of the 2-0 band of $\mathrm{CO}$ at $2.3 \mu \mathrm{m}$; calibration standard frequencies from high resolution color center laser spectroscopy. J. Mol. Spectrosc. 99 (1983) 357-368.

34. W.C. Swan, S.L. Gilbert. Pressure induced shift and broadening of 1510-1540-nm acetylene wavelength calibration lines. J. Opt. Soc. Am. B 17 (2000) 1263-1270.

35. C.E. Miller, L.R. Brown, R.A. Toth, D.C. Benner, V.M. Devi. Spectroscopic challenges for high accuracy retrievals of atmospheric $\mathrm{CO}_{2}$ and the Orbiting Carbon Observatory (OCO) experiment. C.R. Phys. 6 (2005) 876-887.

36. L.S. Rothman, I.E. Gordon, A. Barbe, D.C. Benner, P.F. Bernath, M. Birk, et al. The HITRAN2008 molecular spectroscopic database. J. Quant. Spectrosc. Radiat. Transfer 110 (2009) 533-572. 
37. V.M. Devi, D.C. Benner, L.R. Brown, C.E. Miller, R.A. Toth. Line mixing and speed dependence in $\mathrm{CO}_{2}$ at $6348 \mathrm{~cm}^{-1}$. Positions and intensities, and air- and self-broadening derived with constrained multispectrum analysis. J. Mol. Spectrosc. 242 (2007) 90-117.

38. V.M. Devi, D.C. Benner, M.A.H. Smith, A.W. Mantz, K. Sung, L.R. Brown, et al. (a) Spectral line parameters including temperature dependences of self- and air-broadening in the $2 \leftarrow 0$ band of CO at $2 \mu \mathrm{m}$. J. Quant. Spectrosc. Radiat. Transfer 113 (2012) 1013-1033. (b) Erratum to Spectral line parameters including temperature dependences of self- and airbroadening in the 2-0 band of CO at $2.3 \mu \mathrm{m}$. [J. Quant. Spectrosc. Radiat. Transfer 113 (2012) 1013-1033]. J. Quant. Spectrosc. Radiat. Transfer 116 (2013) 199-200.

39. R.L. Hawkins, M.I. Hoke, J.H. Shaw. Band parameters of $\mathrm{N}_{2} \mathrm{O}$ and $\mathrm{CO}_{2}$ determined by whole band analysis. Proc. Soc. Photo-Opt. Instr. Eng. 289 (1981) 99-201.

40. C.I. Lin, J.H. Shaw. Line parameters determined by spectral curve fitting. J. Opt. Soc. Am. 67 (1977) 1442-1442.

41. C.E. Miller, L.R. Brown. Near infrared spectroscopy of carbon dioxide. I. ${ }^{16} \mathrm{O}^{12} \mathrm{C}^{16} \mathrm{O}$ line positions. J. Mol. Spectrosc. 228 (2004) 329-354.

42. B.M. Elliott, K. Sung, C.E. Miller. FT-IR spectra of ${ }^{17} \mathrm{O}$-enriched $\mathrm{CO}_{2}$ in the $v_{3}$ region: High accuracy frequency calibration and spectroscopic constants for ${ }^{16} \mathrm{O}^{12} \mathrm{C}^{17} \mathrm{O},{ }^{17} \mathrm{O}^{12} \mathrm{C}^{17} \mathrm{O}$, and ${ }^{17} \mathrm{O}^{12} \mathrm{C}^{18}$ O. J. Mol. Spectrosc. 304 (2014) 1-11.

43. Gamache RR [unpublished results, 2015].

44. L.S. Rothman, D. Jacquemart, A. Barbe, D.C. Benner, M. Birk, L.R. Brown, et al. The HITRAN2004 molecular spectroscopic database. J. Quant. Spectrosc. Radiat. Transfer 96 (2005) 139-204.

45. L.S. Rothman, I.E. Gordon, Y. Babikov, A. Barbe, D.C. Benner, P.F. Bernath, et al. The HITRAN2012 molecular spectroscopic database. J. Quant. Spectrosc. Radiat. Transfer 130 (2013) 4-50. 
46. R.B. Wattson, L.S. Rothman. Direct numerical diagonalization: wave of the future. J. Quant. Spectrosc. Radiat. Transfer 48 (1992) 763-780.

47. S.A. Tashkun, V.I. Perevalov, J.-L. Teffo, A.D. Bykov, N.N. Lavrentieva. CDSD-1000, the high-temperature carbon dioxide spectroscopic databank. J. Quant. Spectrosc. Radiat. Transfer 82 (2003)165-196.

48. S.A. Tashkun, V.I. Perevalov, R.R. Gamache. J. Lamouroux. CDSD-296, high resolution carbon dioxide spectroscopic databank: Versions for atmospheric applications. J. Quant. Spectrosc. Radiat. Transfer 152 (2015) 45-73.

49. X. Huang [Private communication, Dec. 2015].

50. E. Zak, J. Tennyson, O.L. Polyansky, L. Lodi, N.F. Zobov, S.A. Tashkun, V.I. Perevalov. A room temperature $\mathrm{CO}_{2}$ linelist with $a b$ initio calculated intensities. J. Quant. Spectrosc. Radiat. Transfer (in press). http://dx.doi.org/10.1016/j.jqsrt.2015.12.022

51. R.R. Gamache, L.S. Rothman. Extension of the HITRAN database to Non-LTE applications. J. Quant. Spectrosc. Radiat. Transfer 48 (1992) 519-525.

52. R.R. Gamache, A. Goldman. Einstein A coefficient, integrated band intensity, and population factors: Application to the $a^{1}{ }_{g} X_{g}^{3}(0,0) \mathrm{O}_{2}$ band. J. Quant. Spectrosc. Radiat. Transfer. 69 (2001) 389-401.

53. C.P. Rinsland, D.C. Benner, V.M. Devi. Absolute line intensities in $\mathrm{CO}_{2}$ bands near $4.8 \mu \mathrm{m}$. Appl. Opt. 25 (1986) 1204-1214.

54. G. Herzberg. Molecular Spectra and Molecular Structure. New York. Van Nostrand Reinhold Company, 1950 (pp. 282-284).

55. A. Baldacci, C.P. Rinsland. M.A.H. Smith, K. Narahari Rao. Spectrum of ${ }^{13} \mathrm{C}^{16} \mathrm{O}_{2}$ at $2.8 \mu \mathrm{m}$. J. Mol. Spectrosc. 94 (1982) 351-362.

56. D. Lisak, A. Cygan, P. Wcislo, R. Ciurylo. Quadratic speed dependence of collisional broadening and shifting for atmospheric applications. J. Quant. Spectrosc. Radiat. Transfer 151 (2015) 43-48. 
57. R.R. Gamache, J. Lamouroux. Predicting accurate line shape parameters for $\mathrm{CO}_{2}$ transitions. J. Quant. Spectrosc. Radiat. Transfer 130 (2013) 158-171.

58. V.M. Devi, D.C. Benner, K. Sung, L.R. Brown, T.J. Crawford, C.E. Miller, et al. Line parameters including temperature dependences of self- and air-broadened line shapes of ${ }^{12} \mathrm{C}^{16} \mathrm{O}_{2}$ : 1.6- $\mu \mathrm{m}$ region. J. Quant. Spectrosc. Radiat. Transfer (in press). http://dx.doi.org/10.1016/j.jqsrt.2015.12.020

59. A. Predoi-Cross, W. Liu, R. Murphy, C. Povey, R.R. Gamache, A.L. Laraia, et al. Measurements and computations for temperature dependences of self-broadened carbon dioxide transitions in the $30012 \leftarrow 00001$ and $30013 \leftarrow 00001$ bands. J. Quant. Spectrosc. Radiat. Transfer 111 (2010) 1065-1079.

60. D. Robert, J. Bonamy. Short range force effects in semi-classical molecular line broadening calculations. J. Phys. 40 (1979) 923-943.

61. A. Predoi-Cross, A.R.W. McKellar, D.C. Benner, V.M. Devi, R.R. Gamache, C.E. Miller, et al. Temperature dependences for air-broadened Lorentz half-width and pressure shift coefficients in the $30013 \leftarrow 00001$ and $30012 \leftarrow 00001$ bands of $\mathrm{CO}_{2}$ near $1600 \mathrm{~nm}$. Can. J. Phys. 87 (2009) 517-535.

62. V.M. Devi, D.C. Benner, K. Sung, T.J. Crawford, Yu Shanshan, L.R. Brown, et al. Self- and Air-broadened Line Shapes in the $2 v_{3} \mathrm{P}$ and R Branches of ${ }^{12} \mathrm{CH}_{4}$. J. Mol. Spectrosc. 315 (2015) $114-136$.

63. A. Vitcu. Line shape studies in the 0310-0110 Q branch of $\mathrm{N}_{2} \mathrm{O}$ using a mid-infrared difference frequency spectrometer. Dissertation, University of Toronto, 2003.

64. J. Lamouroux, H. Tran, A.I. Laraia, R.R. Gamache, L.S. Rothman, I.E. Gordon, J.-M. Hartmann. Updated database plus software for line-mixing in $\mathrm{CO}_{2}$ infrared spectra and their test using laboratory spectra in the $1.5-2.3 \mu \mathrm{m}$ region. J. Quant. Spectrosc. Radiat. Transfer 111 (2010) 2321-2331. 
65. J. Lamouroux, L. Régalia, X. Thomas, J. Vander Auwera, R.R. Gamache, J.-M. Hartmann. $\mathrm{CO}_{2}$ line-mixing database and software update and its tests in the $2.1 \mu \mathrm{m}$ and $4.3 \mu \mathrm{m}$ regions. J. Quant. Spectrosc. Radiat. Transfer 151 (2015) 88-96.

66. J.-M. Hartmann, H. Tran, N.H. Ngo, X. Landsheere, P. Chelin, Y. Lu, et al. Ab initio calculations of the spectral shapes of $\mathrm{CO}_{2}$ isolated lines including non-Voigt effects and comparisons with experiments. Phys. Rev. A. 87 (2013) 013403.

67. H. Tran, N.H. Ngo, J.-M. Hartmann. Efficient computation of some speed-dependent isolated line profiles. J. Quant. Spectrosc. Radiat. Transfer 129 (2013) 199-203.

68. D.A. Long, S. Wó jtewicz, C.E. Miller, J.H. Hodges. Frequency-agile, rapid scanning cavity ring-down spectroscopy (FARS-CRDS) measurements of the $(30012) \longleftarrow(00001)$ near infrared carbon dioxide band. J. Quant. Spectrosc. Radiat. Transfer 161 (2015) 35-40.

69. B.J. Connor, H. Boesch, G. Toon, B. Sen, C.E. Miller, D. Crisp. Orbiting Carbon Observatory: Inverse method and prospective error analysis. J. Geophys. Res. 113 (2008) D05305, doi: 10.1029/2006JD008336, 2008. 
Table titles

1. Summary of line shape measurements ${ }^{\mathrm{a}}$ reported for $\mathrm{CO}_{2}$ bands near $2 \mu \mathrm{m}$.

2. Experimental setups and physical conditions of analyzed $\mathrm{CO}_{2}$ spectra.

3. Summary of experimental conditions of spectra analyzed.

4. Major bands included in the $2 \mu \mathrm{m}$ region $^{\mathrm{a}}$ of the fitted interval.

5. Measured line parameters and number of $\mathrm{CO}_{2}$ measurements.

6. (a) Ro-vibrational constants, vibrational band strengths $\left(S_{\mathrm{v}}\right)$, and correlation coefficients of measured $\mathrm{CO}_{2}$ bands: $4700-4930 \mathrm{~cm}^{-1}$. (b) Correlation coefficients for the upper state rovibrational constants for the $21113 \leftarrow 01101 \mathrm{e}, 21113 \leftarrow 01101 \mathrm{f}$ and the $40002 \longleftarrow 10002$ interacting bands of ${ }^{12} \mathrm{C}^{16} \mathrm{O}_{2}$.

7. Comparisons of vibrational band strengths, $S_{\mathrm{v}}(\mathrm{cm} / \mathrm{molecule})$ at $296 \mathrm{~K}$, for select bands ${ }^{\mathrm{a}}$ measured in the $2-\mu \mathrm{m}$ region of $\mathrm{CO}_{2}$.

8. A sample of measured line parameters for the P20-R20 transitions in the $20013 \longleftarrow 00001$ band of ${ }^{12} \mathrm{C}^{16} \mathrm{O}_{2}$.

9. Measured off-diagonal relaxation matrix element coefficients, $W_{\mathrm{ij}}\left(\mathrm{cm}^{-1} \mathrm{~atm}^{-1}\right)$ at $296 \mathrm{~K}$, and their temperature dependence exponents for $\mathrm{CO}_{2}-\mathrm{CO}_{2}$ and $\mathrm{CO}_{2}$-air for the $20013 \leftarrow 00001$ band of ${ }^{12} \mathrm{C}^{16} \mathrm{O}_{2}$. 


\section{Figure captions}

Fig. 1. Five laboratory absorption spectra $\left(4700-4930 \mathrm{~cm}^{-1}\right)$ of pure $\mathrm{CO}_{2}$ and dilute mixtures of $\mathrm{CO}_{2}$ in air in the $20013 \leftarrow 00001$ region recorded at high resolution $\left(0.004-0.012 \mathrm{~cm}^{-1}\right)$ using two different FTS. Spectra shown in (a) and (b) were recorded using the Kitt Peak FTS with a natural sample of $\mathrm{CO}_{2}$ and a $99 \%{ }^{13} \mathrm{C}$-enriched $\mathrm{CO}_{2}$ near room temperature. Spectra shown in panels (c)(d) were recorded at JPL with a Bruker IFS-125HR FTS using a $99.99 \%{ }^{12} \mathrm{C}$-enriched $\mathrm{CO}_{2}$ and $\mathrm{CO}_{2}$ +air: (c) Pure $\mathrm{CO}_{2}$ at $170.3 \mathrm{~K}$, (d) $0.051 \% \mathrm{CO}_{2}$ in air at $295.7 \mathrm{~K}$ and (e) $\sim 0.03 \% \mathrm{CO}_{2}$ in air at 169.8 K. (For interpretation of the reference to color in this figure legend, the reader is referred to the electronic version of this article).

Fig. 2. The multispectrum fit of the $4700-4930 \mathrm{~cm}^{-1}$ region covering the strongest $20013 \leftarrow 00001$ band of ${ }^{12} \mathrm{C}^{16} \mathrm{O}_{2}$ and a number of other bands of $\mathrm{CO}_{2}$. All 27 experimental spectra are overlaid in the top panel (a) and the weighted observed minus calculated $(\mathrm{O}-\mathrm{C})$ fit residuals are plotted in the lower panel (b). The dashed horizontal line in (a) corresponds to the $100 \%$ absorption level. Color codes: (Refer Table 3) Black: (self-broadened); Red: (air-broadened); Blue: $\left({ }^{13} \mathbf{C}^{16} \mathbf{O}_{2}\right)$. (For interpretation of the reference to color in this figure legend, the reader is referred to the electronic version of this article).

Fig. 3. This figure is a re-plot of Fig. 2 with the weighted fit residuals grouped according to different ranges of the sample temperatures. Similar to Fig. 2, the top panel (a) displays all the 27 spectra fitted simultaneously. Panel (b) shows the fit residuals from all 27 spectra at various pressures, temperatures and volume mixing ratios of $\mathrm{CO}_{2}$; (c) shows the two $99 \%{ }^{13} \mathrm{C}$-enriced $\mathrm{CO}_{2}$ at room temperature; (d) has two pure ${ }^{12} \mathrm{CO}_{2}$ spectra at 170 and $180 \mathrm{~K}$, and (e) shows five air-broadened $\mathrm{CO}_{2}$ spectra between 170 and $195 \mathrm{~K}$ (see Table 3).

Fig. 4. The comparisons of line position differences $\left(\mathrm{cm}^{-1}\right)$ plotted vs. $m$ (where $m=-J^{\prime \prime}, J^{\prime \prime}+1$ for P- and R-branch lines of the 20013-00001 band of ${ }^{12} \mathrm{C}^{16} \mathrm{O}_{2}$, respectively): Present study [PS]HITRAN2012 [45] open black circles, PS-Toth et al. [11] open green diamonds, PSHITRAN2004 [44] solid blue triangles, and PS-HITRAN2008 [36] solid red stars. If line positions from PS are compared to only P58-R58 transitions measured in Toth et al. [11], the means and standard deviations for all four comparisons shown above would very nearly be the same: $0.0001716(8) \mathrm{cm}^{-1}$. (For interpretation of the reference to color in this figure legend, the reader is referred to the electronic version of this article). 
Fig. 5. Line intensities (cm/molecule at $296 \mathrm{~K})$ in natural abundance determined for the $P$ and $R$ branches for the $20013 \leftarrow 00001$ band of ${ }^{12} \mathrm{C}^{16} \mathrm{O}_{2}$ plotted as a function of $m$ ( $m=-J$ " for P-branch and $J^{\prime \prime}+1$ for R-branch lines). A linear scale is used in (a) and a logarithmic scale in (b). In (b) the line intensities and their uncertainties are plotted. In panel (c), ratios of line intensities between PS/HITRAN [36,44,45]; and PS/Toth et al. [11] are plotted. The error bars in (c) are dominated by the uncertainties in Toth et al. [11]. The reason for the discontinuity observed near $\mathrm{P}(58)$ and $\mathrm{R}(60)$ may be due to some difficulties in merging intensities beyond Toth et al. [11] experimental values and other calculated intensities $[\mathbf{4 5 , 4 8}]$. (For interpretation of the reference to color in this figure legend, the reader is referred to the electronic version of this article).

Fig. 6(a). The effect on line positions due to crossing interaction (due to Fermi resonance) in the $21103 \leftarrow 01101$ and $40002 \leftarrow 01101$ bands of ${ }^{12} \mathrm{C}^{16} \mathrm{O}_{2}$. The shift in the position of each spectral line as a function of $J^{\prime}$ is plotted. The shift in position at $J^{\prime}=0$ is zero but grows rapidly as a function of $J^{\prime}\left(J^{\prime}+1\right)$ but turns around near $J^{\prime}=50$ at a shift of about $0.15 \mathrm{~cm}^{-1}$. See the text for details.

Fig. 6(b). The fraction of intensity borrowed from the stronger band $(21103 \leftarrow 01101)$ to the weaker band $(40002 \leftarrow 01101)$ of ${ }^{12} \mathrm{C}^{16} \mathrm{O}_{2}$ plotted vs. $J$ (see the text for details).

Fig. 7. (a) The measured Lorentz air- and self-broadened half-width coefficients $\left(\mathrm{cm}^{-1} \mathrm{~atm}^{-1}\right.$ at $296 \mathrm{~K}$ ) for the $20013 \leftarrow 00001$ band of ${ }^{12} \mathrm{C}^{16} \mathrm{O}_{2}$ plotted as a function of $m(m=-J$ " for P-branch and $J^{\prime \prime}+1$ for R-branch lines). The half-width coefficients are compared to those by Toth et al. [11,12], HITRAN2012 [45] and Régalia-Jarlot et al. [18] and the predicted air- and self-width coefficients by Gamache and Lamouroux [57]. The ratios of air-broadened widths from PS to Toth et al. [13] and for self-broadened widths from PS to Toth et al. [12] are plotted in (b) and (c), respectively. The ratios of self- to air-broadened half-width coefficients from PS, Toth et al. [12, 13] and HITRAN [45] are plotted in panel (d). Where error bars are not visible the uncertainties are smaller than the size of the symbols used. The means and standard deviations of the ratios are also given, when appropriate.

Fig. 8. Measured temperature dependence exponents for the air- and self-broadened half-width coefficients ( $n_{1}$ and $n_{2}$, respectively) for the $20013 \leftarrow 00001$ band of ${ }^{12} \mathrm{C}^{16} \mathrm{O}_{2}$ as a function of $m$. Upper panel: (a) Measured $n_{2}$ (red solid up triangles) and $n_{1}$ (solid blue down triangles). The 
values for $n_{2}$ are from Suarez and Valero (green open circles] for the $20012 \leftarrow 00001$ band of ${ }^{12} \mathrm{C}^{16} \mathrm{O}_{2}$. Middle panel: (b) Measured $n_{2}$ (red solid stars) and $n_{1}$ (solid blue stars) for the $30013 \leftarrow 00001$ band of ${ }^{12} \mathrm{C}^{16} \mathrm{O}_{2}$ [58]. In the bottom panel: (c) The predicted $n_{2}$ (red semi-filled squares) and $n_{1}$ (blue semi-filled circles) from Gamache and Lamouroux [57]. The horizontal black dashed line in all three panels corresponds to the temperature dependence exponent $n=$ 0.75 (default values normally used). (For interpretation of the reference to color in this figure legend, the reader is referred to the electronic version of this article).

Fig. 9. Measured air- and self- pressure-shift coefficients, $\delta^{0}$ (air) and $\delta^{0}$ (self), and their temperature dependences $\delta^{\prime}$ (air) and $\delta^{\prime}$ (self), respectively, are plotted in panels (a)-(c). In (a) the air- and self-shift coefficients from PS, Toth et al. [12,13], and HITRAN [45] are shown; in the middle panel (b) the temperature dependences $\delta^{\prime}$ (air) and $\delta^{\prime}$ (self) for the $20013 \leftarrow 0001$ band is plotted; in the bottom panel (c) the temperature dependences $\delta^{\prime}$ (air) and $\delta^{\prime}$ (self) for the $30013 \leftarrow 00001$ [57] are shown for comparisons. The horizontal black dashed line in (b) and (c) represents zero temperature dependence. (For interpretation of the reference to color in this figure legend, the reader is referred to the electronic version of this article).

Fig. 10. (a) Measured off-diagonal relaxation matrix element coefficients, $W_{i j},\left(\mathrm{~cm}^{-1} \mathrm{~atm}^{-1}\right)$ at $296 \mathrm{~K}$ for $\mathrm{CO}_{2}-\mathrm{CO}_{2}$ and $\mathrm{CO}_{2}$-air plotted vs. $m\left(m=-J^{\prime \prime}\right.$ for P branch and $J^{\prime \prime}+1$ for R-branch lines) for the $20013 \leftarrow 00001$ band of ${ }^{12} \mathrm{C}^{16} \mathrm{O}_{2}$ and comparison to the corresponding room temperature measurements [16], (b) The measured speed dependence parameter in the $\mathrm{P}$ and $\mathrm{R}$ branches for the $20013 \leftarrow 00001$ band plotted against $m$. from present study and [16, indicated here by green **]. Where error bars are not visible, the uncertainties are smaller than the plot symbol. (For interpretation of the reference to color in this figure legend, the reader is referred to the electronic version of this article).

Fig. 11. Influence of line mixing in fitting the laboratory spectra plotted vs. wavenumber $\left(\mathrm{cm}^{-1}\right)$. The fit was performed with a Voigt line shape with quadratic speed dependence and no line mixing in the multispectrum fit. (a) One spectrum $\left(5.1 \% \mathrm{CO}_{2}\right.$ with a total pressure of 649.73 Torr, $20.941 \mathrm{~m}$ path and $\mathrm{T}=220.2 \mathrm{~K}$ ) plotted from the simultaneous fit of 27 spectra. (b) The weighted observed minus calculated fit residuals for the spectrum shown in (a). The characteristic fit residuals associated with line mixing are apparent. (c) The same spectrum in (a) with relaxation matrix elements included in the fit. Notice the improvement in the fit residuals. 
Fig. 12. Uncertainties in the fitted line positions $\left(\mathrm{cm}^{-1}\right)$ are plotted as a function of $J^{\prime}$ for the $20013 \leftarrow 00001$ band of ${ }^{12} \mathrm{C}^{16} \mathrm{O}_{2}$. With the multispectrum fitting and using the theoretical quantum mechanical formulae, very precise determination of line positions are achieved. At $J^{\prime}=40,60$ and 80 , for example, the obtained position uncertainties are nearly $1 \times 10^{-6}, 1 \times 10^{-5}$, and $1 \times 10^{-4}\left(\mathrm{~cm}^{-1}\right)$, respectively.

Fig. 13. The percent intensity uncertainties (ratio of intensity uncertainty to intensity $\times 100$ ) plotted as a function of $m(-J "$ and $J "+1$ for the $\mathrm{P}$ and $\mathrm{R}$ branches, respectively) for the $20013 \leftarrow 00001$ band of ${ }^{12} \mathrm{C}^{16} \mathrm{O}_{2}$ for $\mathrm{P}$ and $\mathrm{R}$ branches. See the text for details.

Fig. 14. Uncertainty in measured Lorentz half-width coefficients $\left(\mathrm{cm}^{-1} \mathrm{~atm}^{-1}\right)$ plotted as a function of gas sample temperature (in $\mathrm{K}$ ) for the $20013 \leftarrow 00001$ band of ${ }^{12} \mathrm{C}^{16} \mathrm{O}_{2}$. The graphs are plotted for select $J$ transitions for both self- and air-broadening. These plots illustrate that the minimum uncertainty in measured half-width coefficients does not always correspond to $\mathrm{T}=296$ $\mathrm{K}$ (where half-width coefficients are usually reported). The inset lists the various transitions for which the graphs are shown. To avoid confusion only P4 self and R42 air are labeled. The dashed curves signify the importance of correlations in reducing the uncertainties in measured half-width coefficients.

Fig. 15. Relative uncertainty (ratios of half-width uncertainty to half-width coefficients) are plotted vs. gas sample temperature (in K) for the same set of transitions shown in Fig. 14. The relative uncertainties with and without considering correlations among fitted parameters are shown by dashed curves. As in Fig. 14, to minimize the congestion, only few lines are labeled.

Fig. 16. Uncertainty of self- and air- pressure-shift coefficients $\left(\mathrm{cm}^{-1} \mathrm{~atm}^{-1}\right)$ plotted as a function of gas sample temperature (in $\mathrm{K}$ ) for $20013 \leftarrow 00001$ band of ${ }^{12} \mathrm{C}^{16} \mathrm{O}_{2}$ illustrating that the minimum uncertainty does not always correspond to $\mathrm{T}=296 \mathrm{~K}$ (where pressure shift coefficients are reported). The curves represent uncertainties for both self- and air-shift coefficients for P18, P42, R4, and R18 transitions. Uncertainties in self-shift coefficients are rather higher than for airshift coefficients (perhaps related to the large $\mathrm{CO}_{2}-\mathrm{CO}_{2}$ mixing observed in self broadening). Plots for only a few $J$-transitions are labeled. Minimum uncertainty occurs generally at lower gas temperatures than at $296 \mathrm{~K}$. 
Fig. 17. Uncertainty in the measured off-diagonal relaxation matrix element coefficients $\left(\mathrm{cm}^{-1}\right.$ $\mathrm{atm}^{-1}$ ) are plotted vs. gas sample temperature (in $\mathrm{K}$ ). Plots are made for two transition pairs, $\mathrm{P} 12 \& \mathrm{P} 14$ and R12\&R14 (for $\mathrm{CO}_{2}-\mathrm{CO}_{2}$ and $\mathrm{CO}_{2}$-air) as marked in the panel. Uncertainties without and with correlations are displayed as a function of temperature to illustrate the importance of correlations in reducing the uncertainty.

Fig. 18. Line intensities from present study are compared to the only other extensive experimental measurements by Toth et al. [11], calculated intensities from the latest HITRAN2012 database [45] and the most recent ab initio calculations by Zak et al. [50]. (a) Line intensities vs. $m$. (b) Line position differences between PS and Zak et al. (c) The line intensity ratios for the same set as in (a) plotted as a function of $m$. (For interpretation of the reference to color in this figure legend, the reader is referred to the electronic version of this article).

Fig. 19. (a) Comparison of line position differences between HITRAN2012 [45] and CDSD296 [48] vs. $m$. (b) Percent intensity differences between HITRAN2012 and CDSD-296 vs. $m$. In (a) and (b), the CDSD-296 data points for P58-P2 and R0-R62 (open black circles and open black triangles, respectively) were calculated using the experimental values from Toth et al. [11] and for the higher $m$ values (solid red stars and solid brown circles, respectively) from Refs. [48] (See the text for details). (For interpretation of the reference to color in this figure legend, the reader is referred to the electronic version of this article).

Fig. 20. (a) Ratios of line intensities: PS/HITRAN2012; PS/Toth et al. [11]; PS/HITRAN2008; PS/Zak et al. [50]; PS/CDSD-296 [48]; PS/Huang [49], (b) Ratios of line intensities between Zak et al./CDSD-296, Zak et al./Huang, and CDSD-296/Huang, (c) Line position differences PS-Zak et al. and PS-CDSD-296, (d) Ratios of Lorentz air- and self-broadened half-width coefficients between PS and CDSD-296, (e) Temperature dependence exponents of Lorentz airbroadened half-width coefficients from PS and CDSD-296, and (f) air- pressure-shift coefficients from PS and CDSD-296. See the text for details. (For interpretation of the reference to color in this figure legend, the reader is referred to the electronic version of this article). 

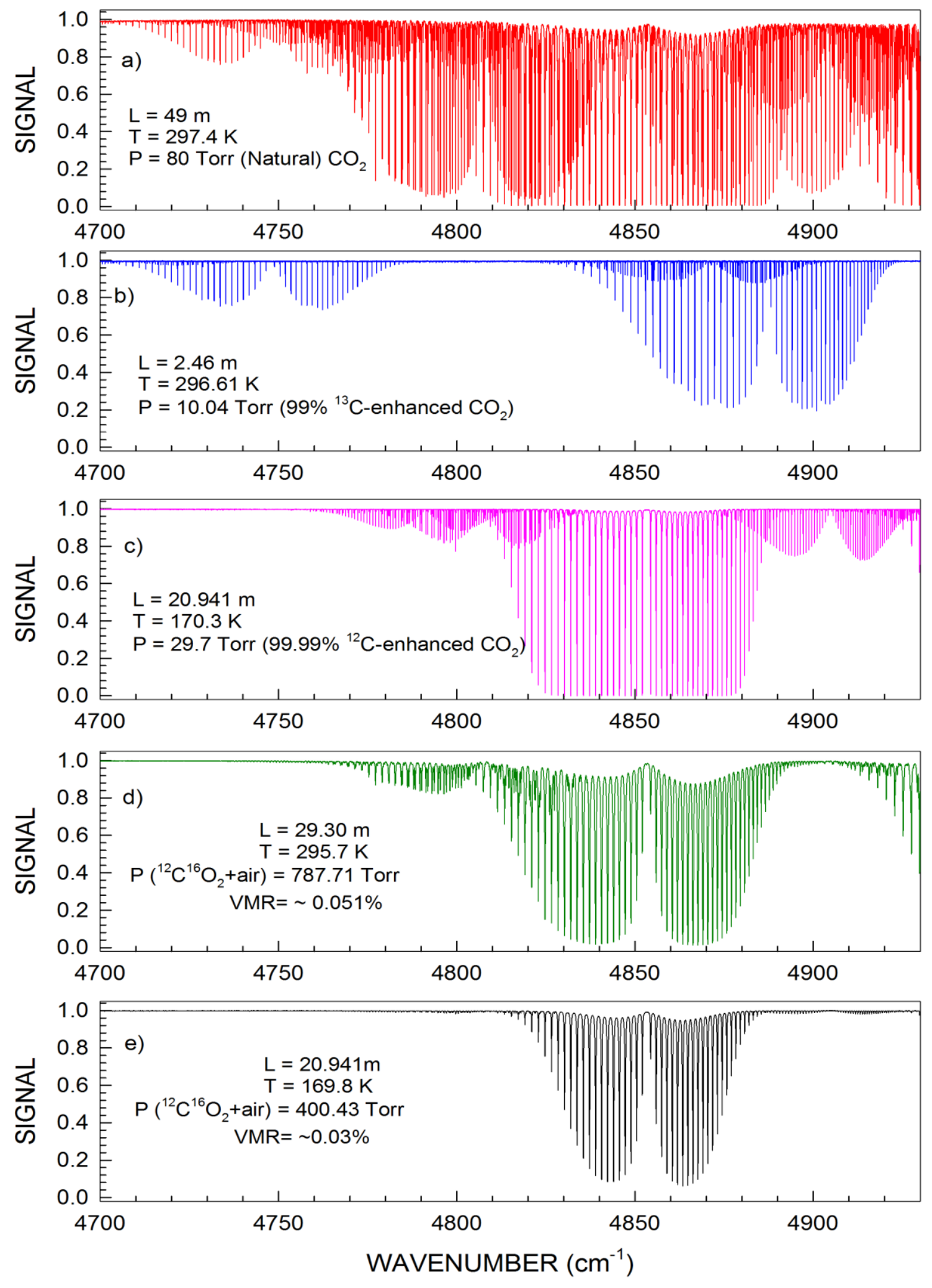

Fig. 1 
a)

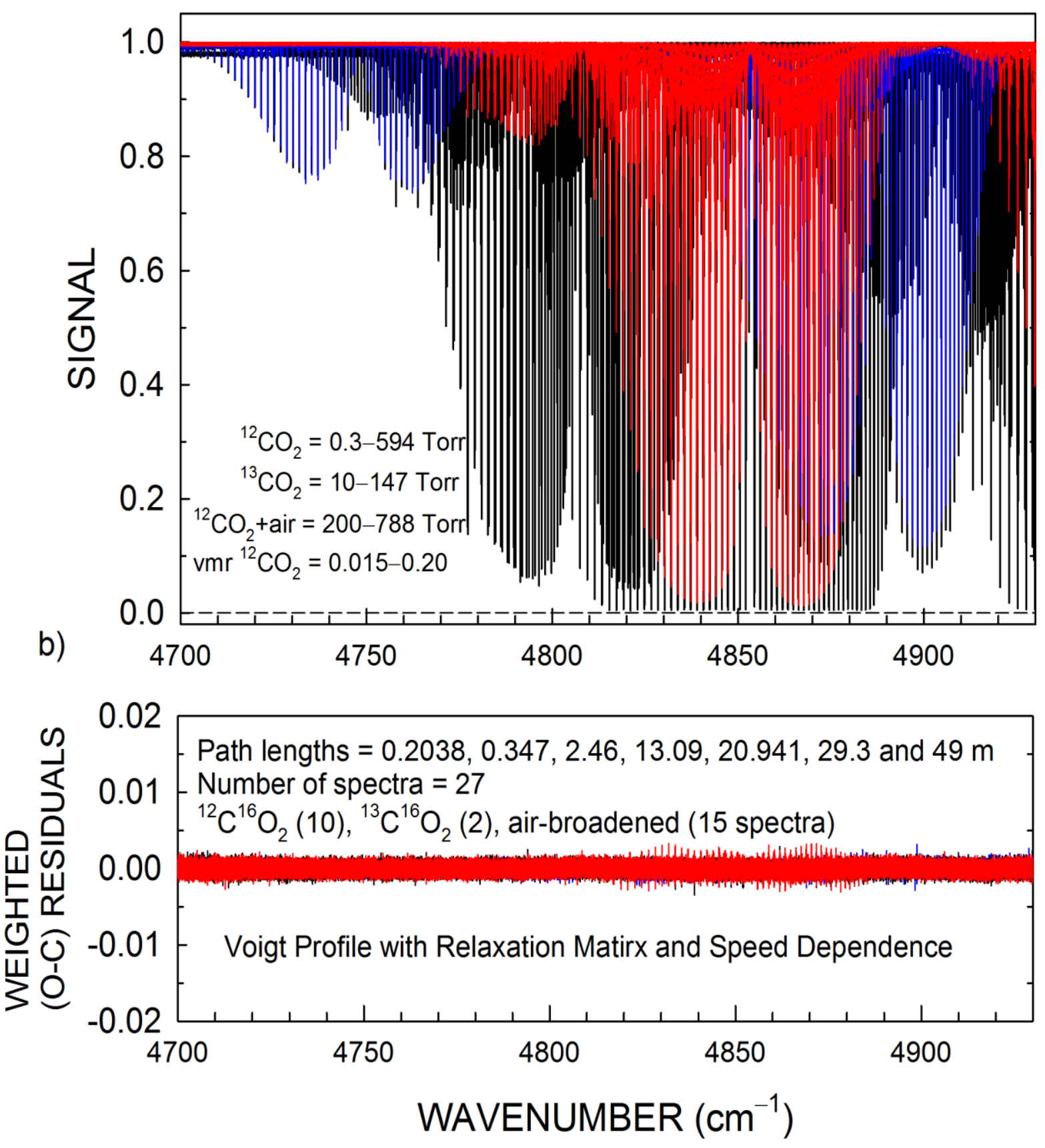

Fig. 2 


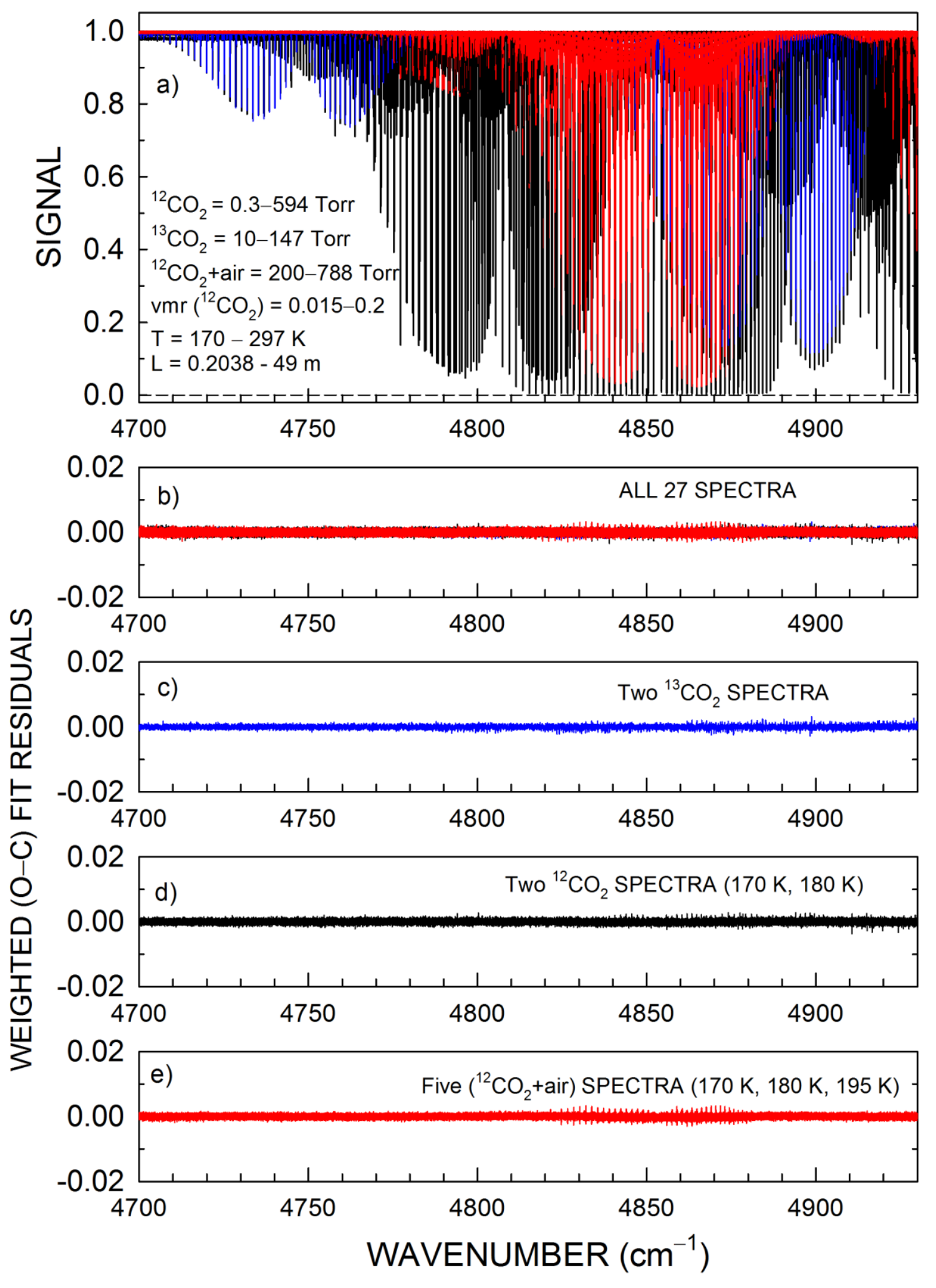

Fig. 3 


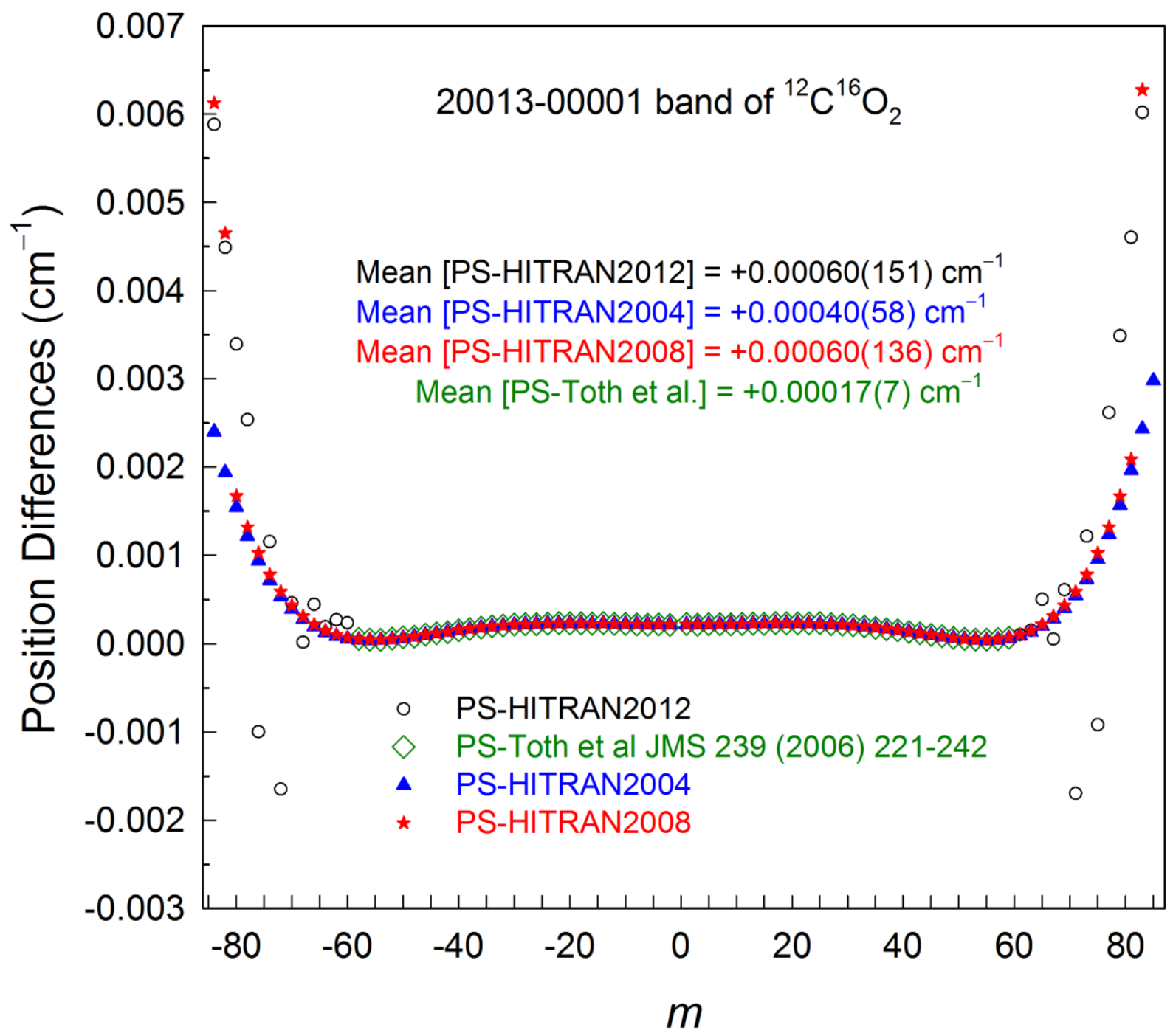

Fig. 4 
a)
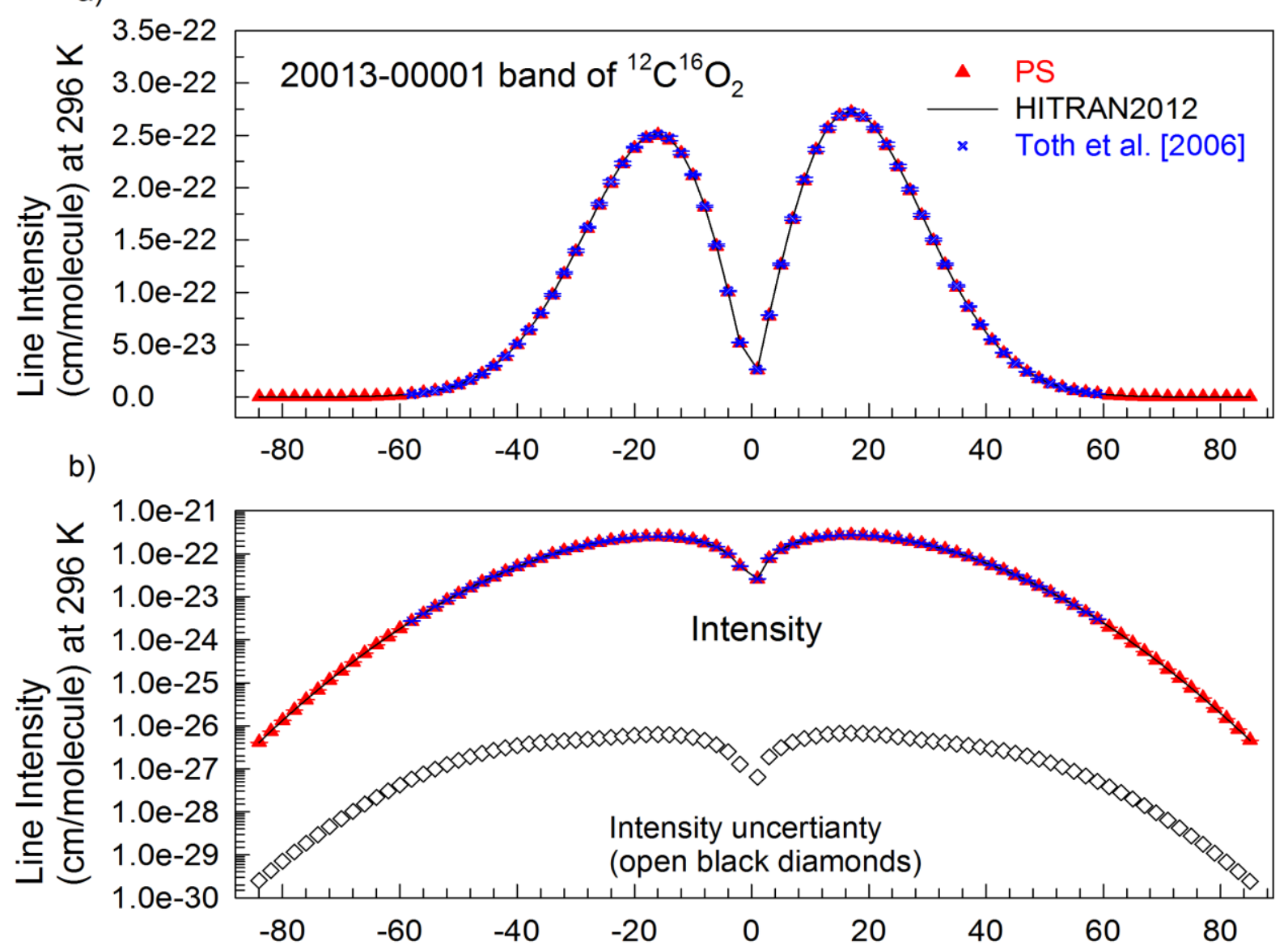

c)

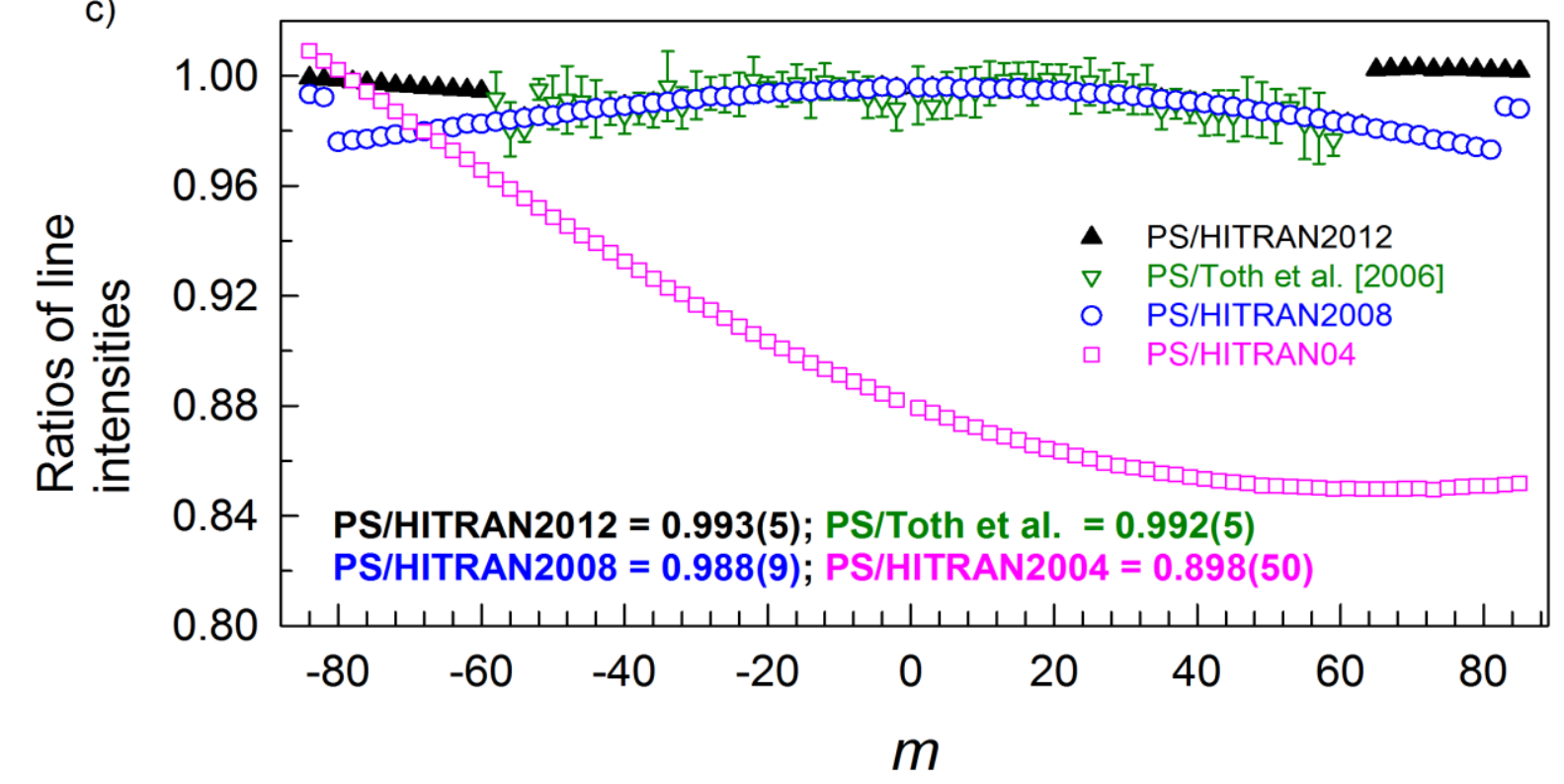

Fig. 5 
a)

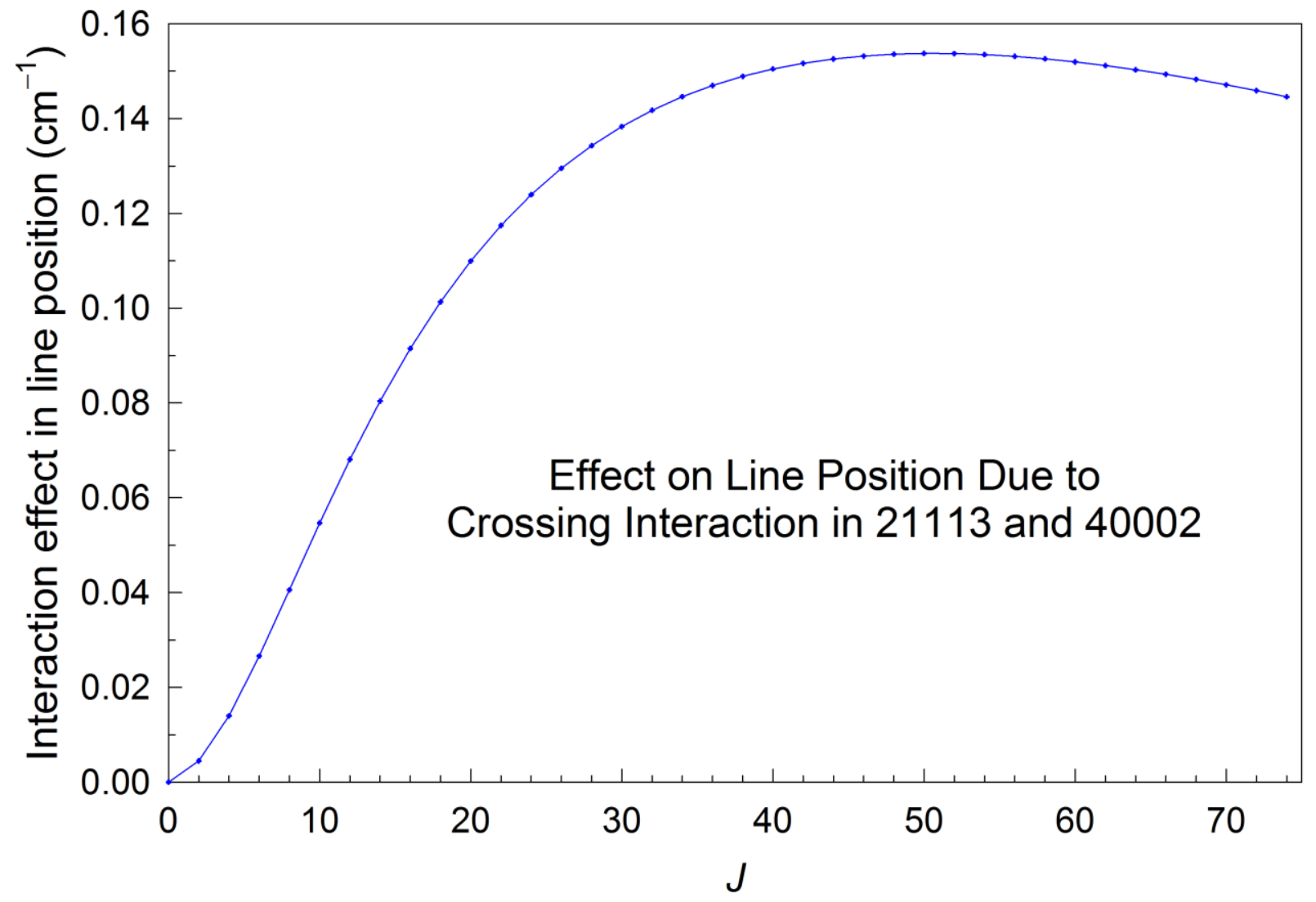

Fig. (6a) 
b)

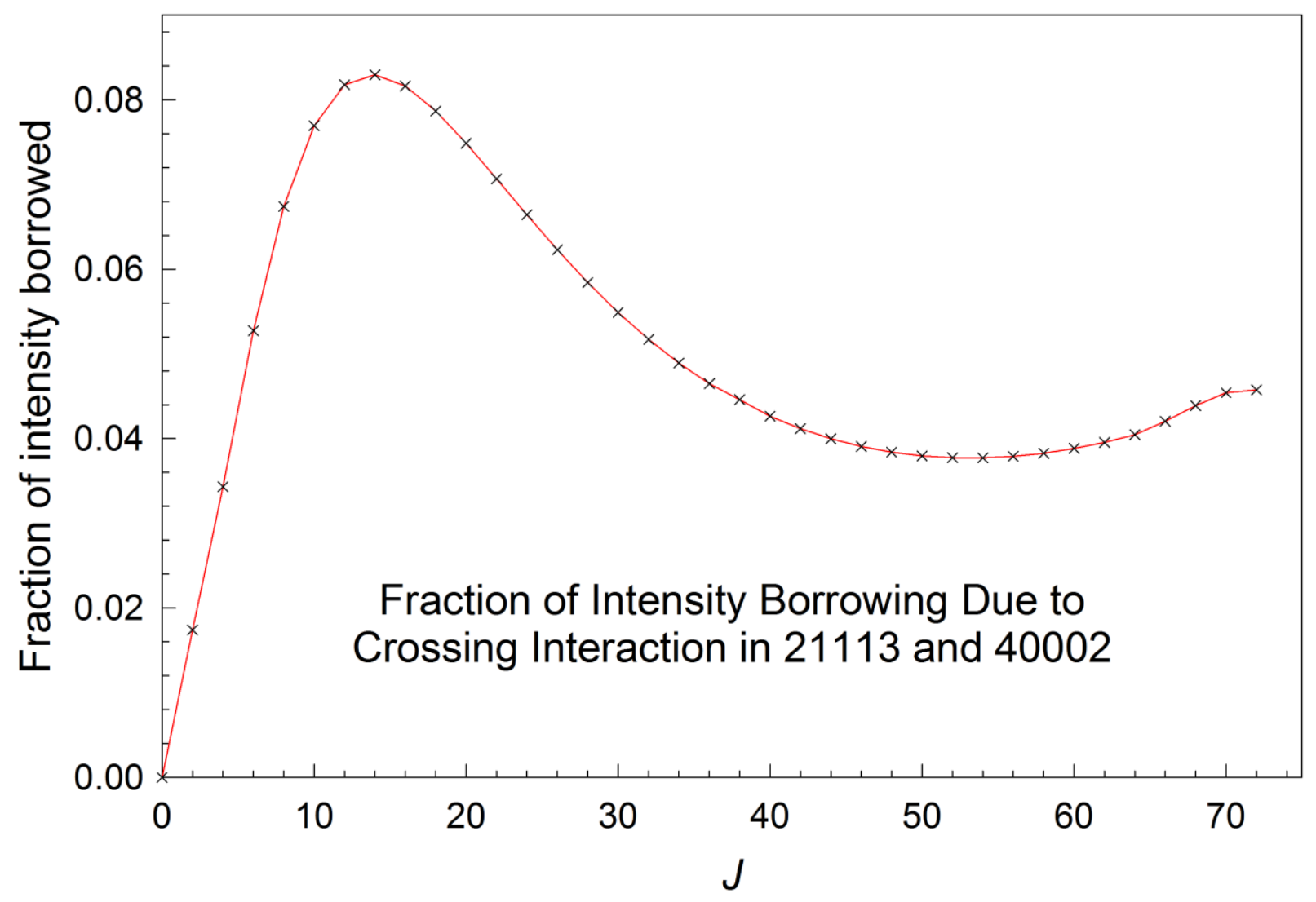

Fig. 6(b) 
a)

b)
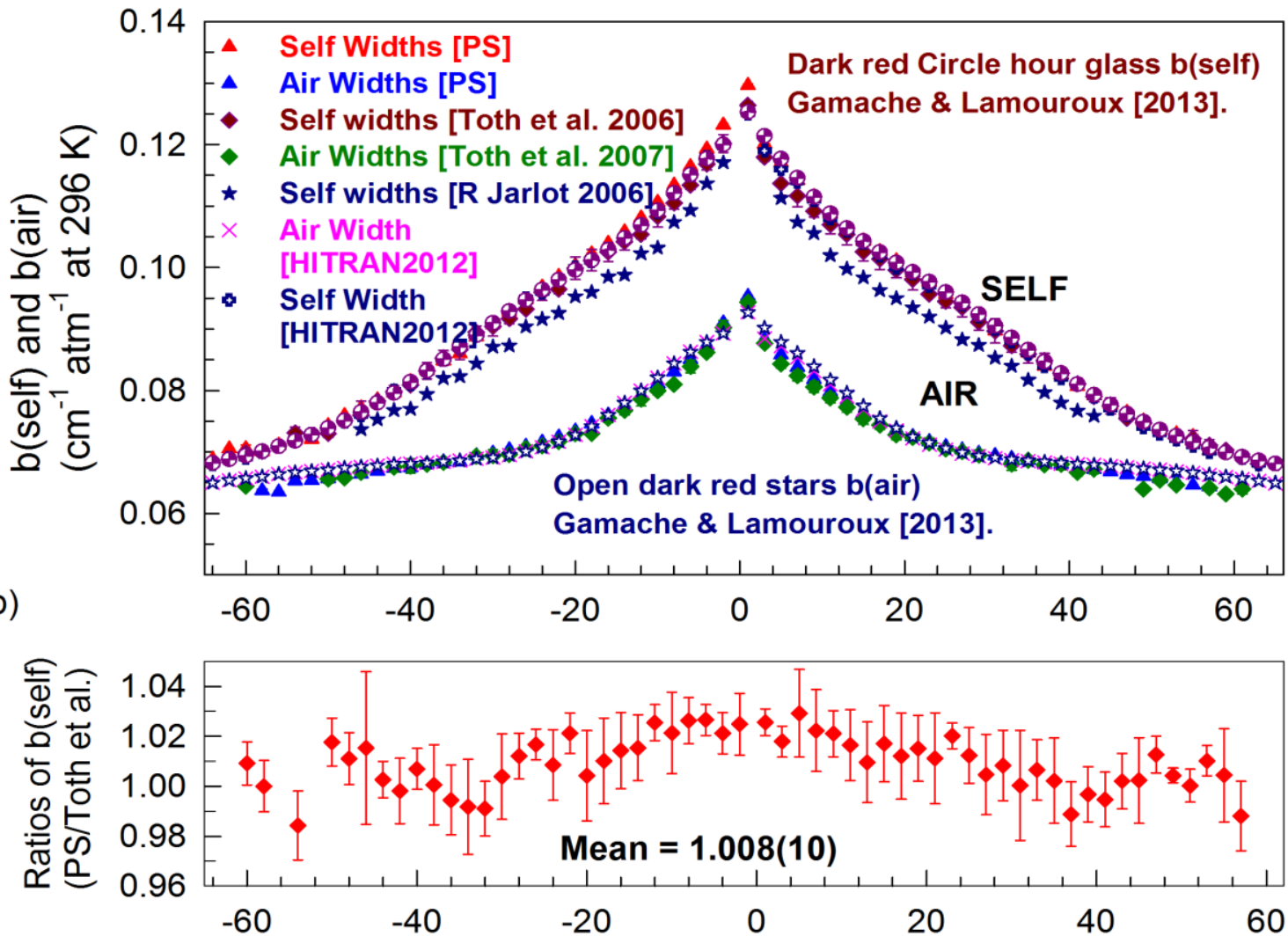

c)

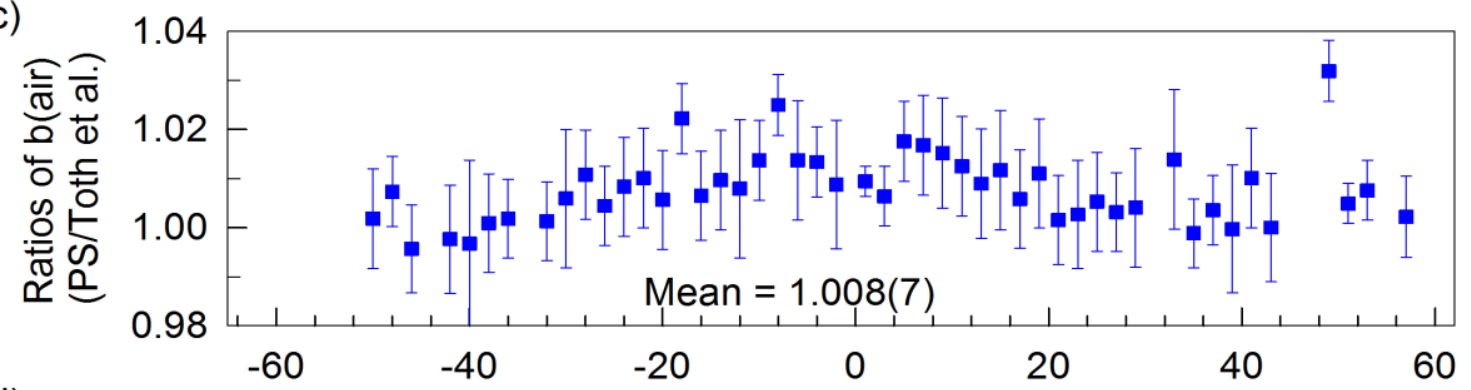

d)

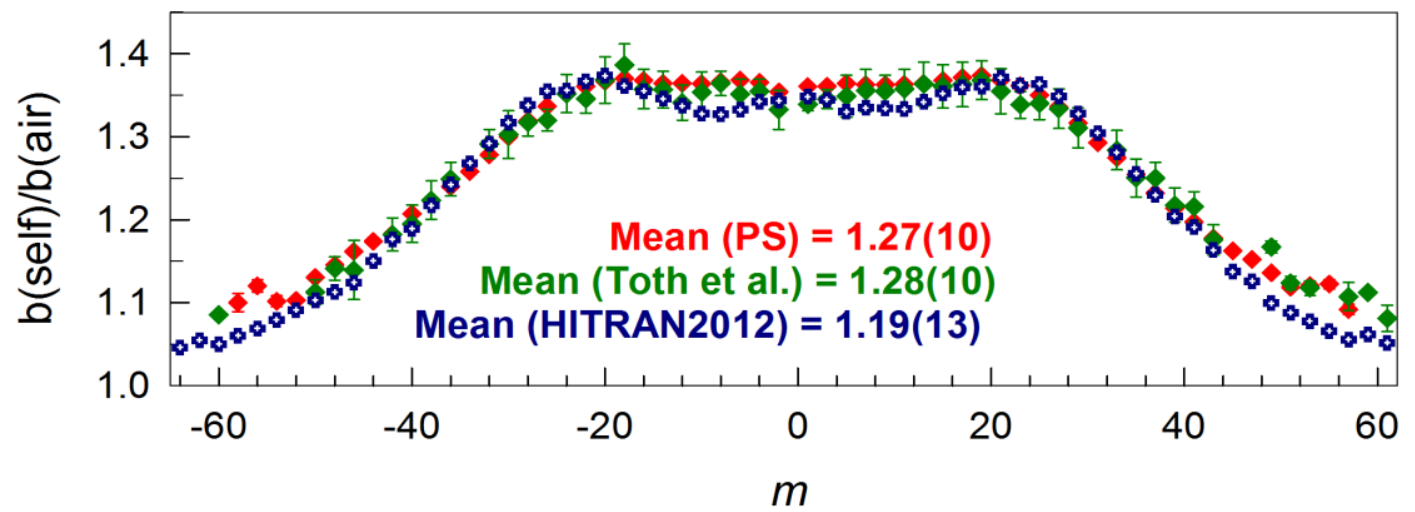

Fig. 7 
a)
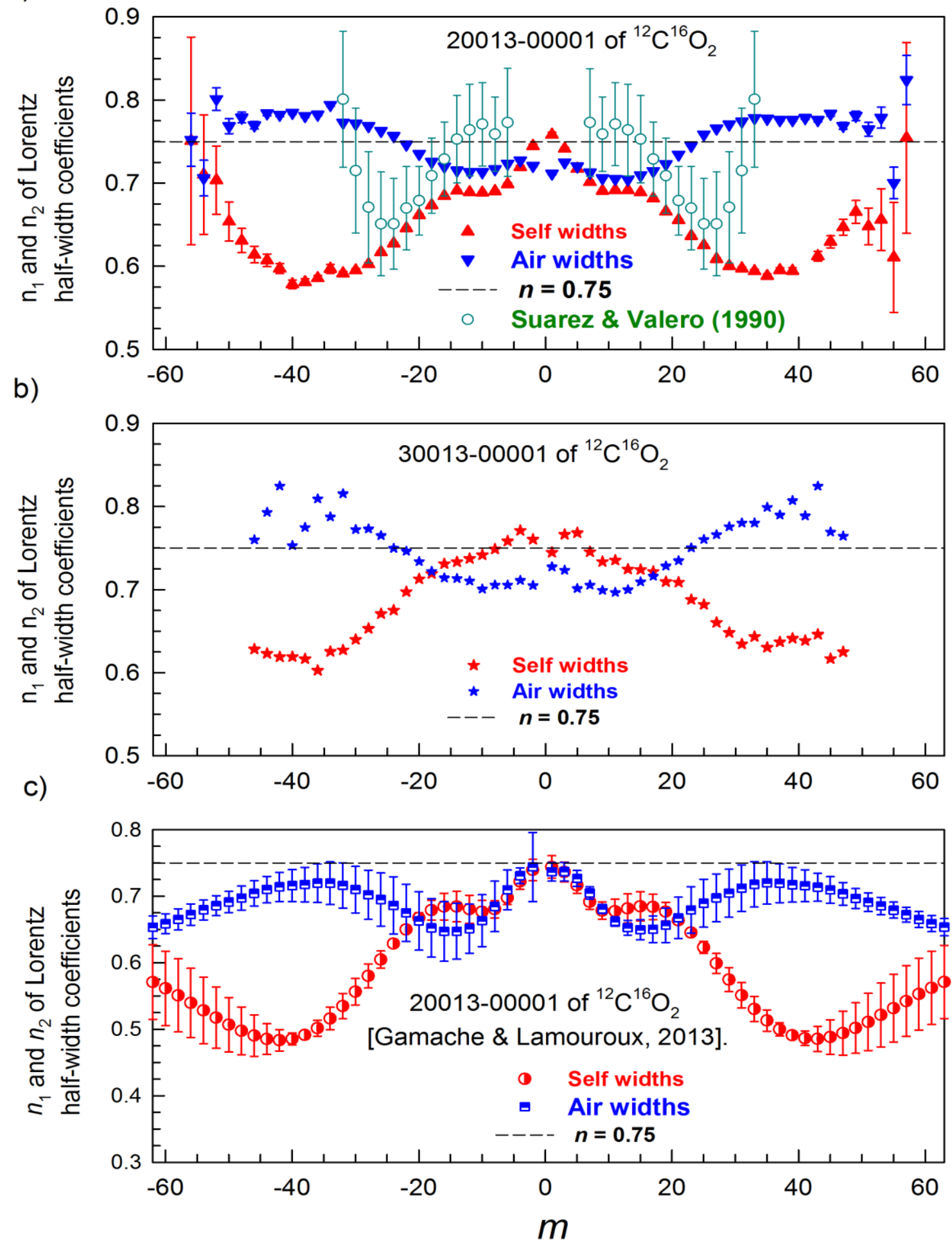

Fig. 8 
a)
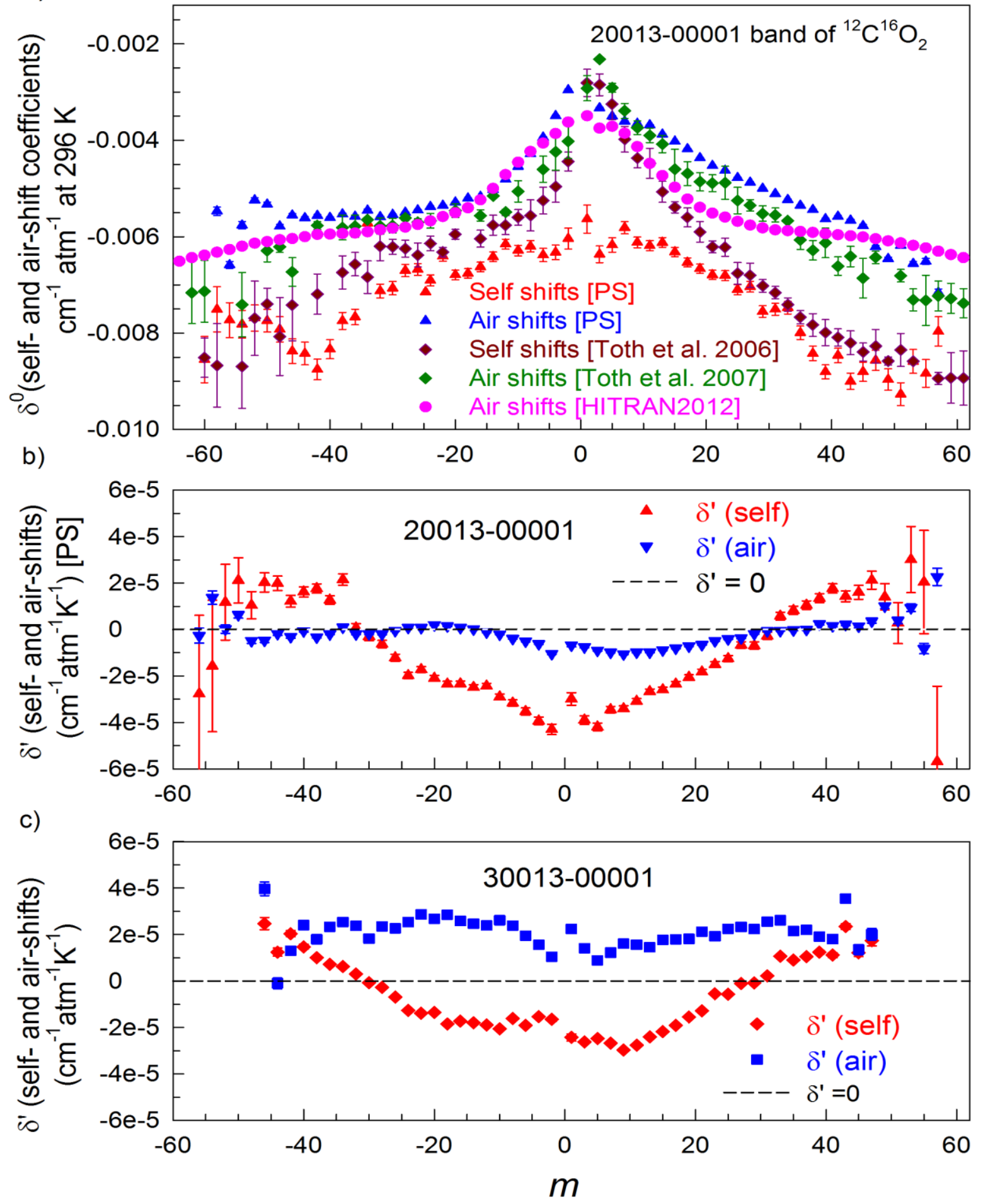

Fig. 9 
(a)

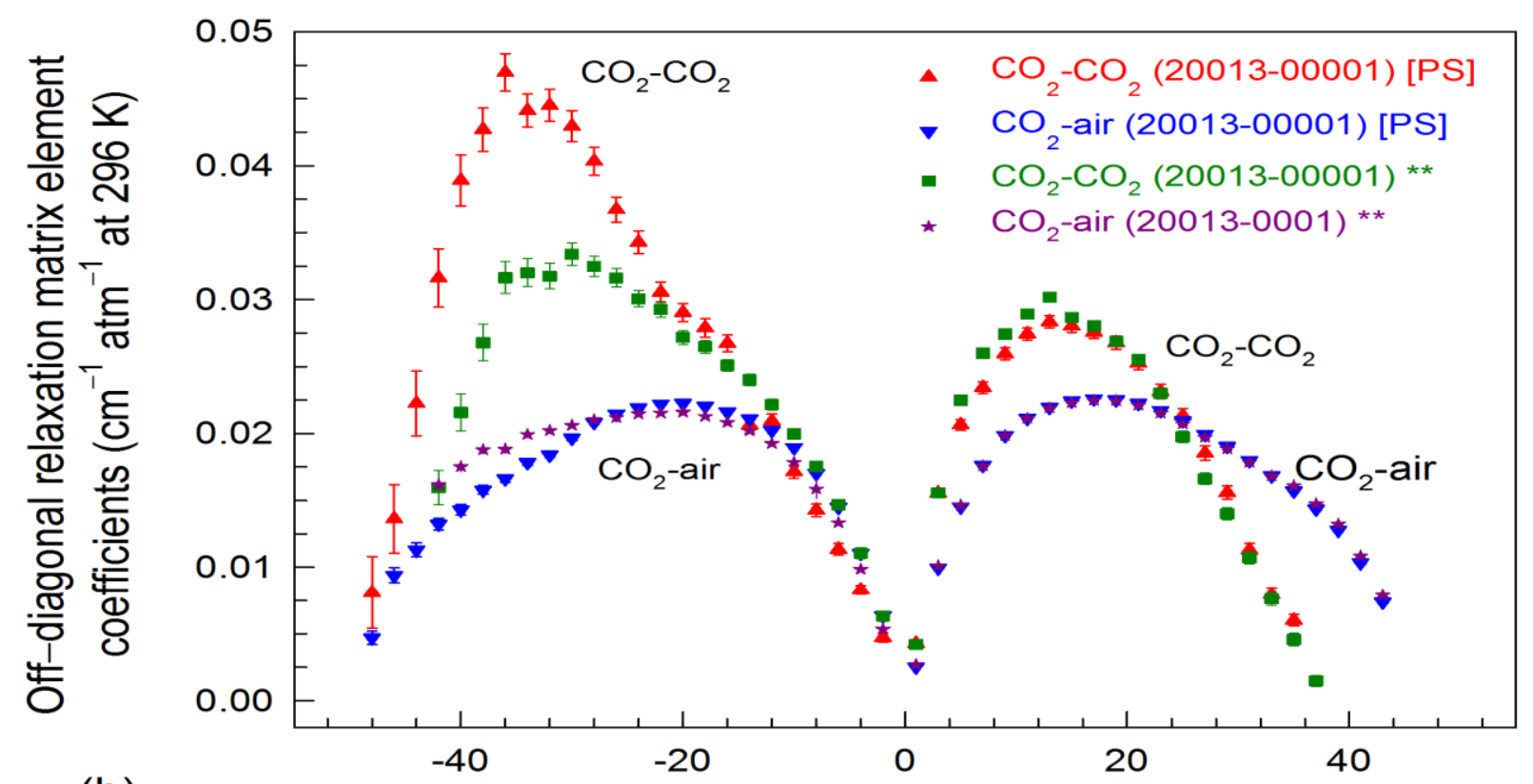

(b)

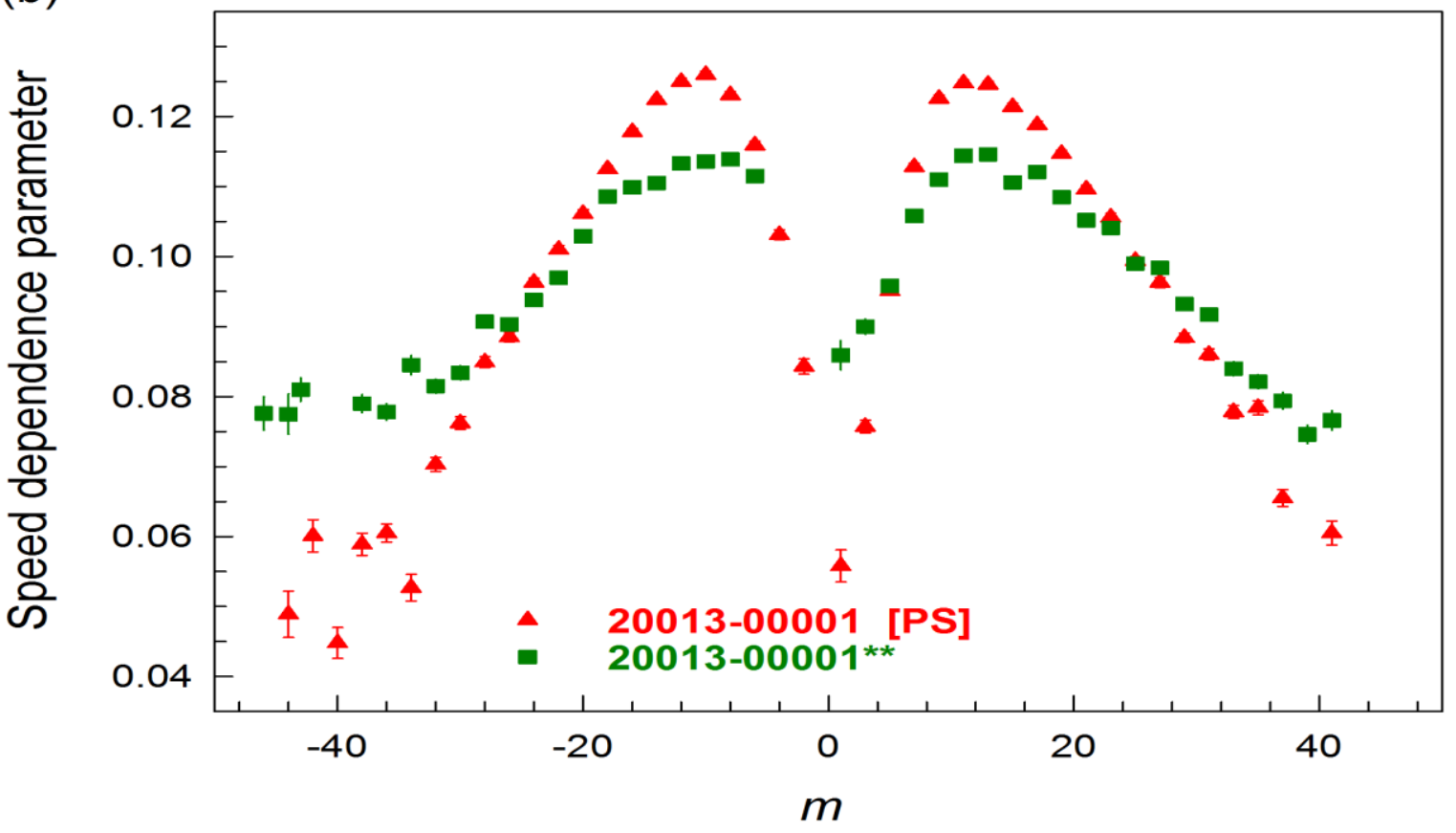

Fig. 10 
a)

b)
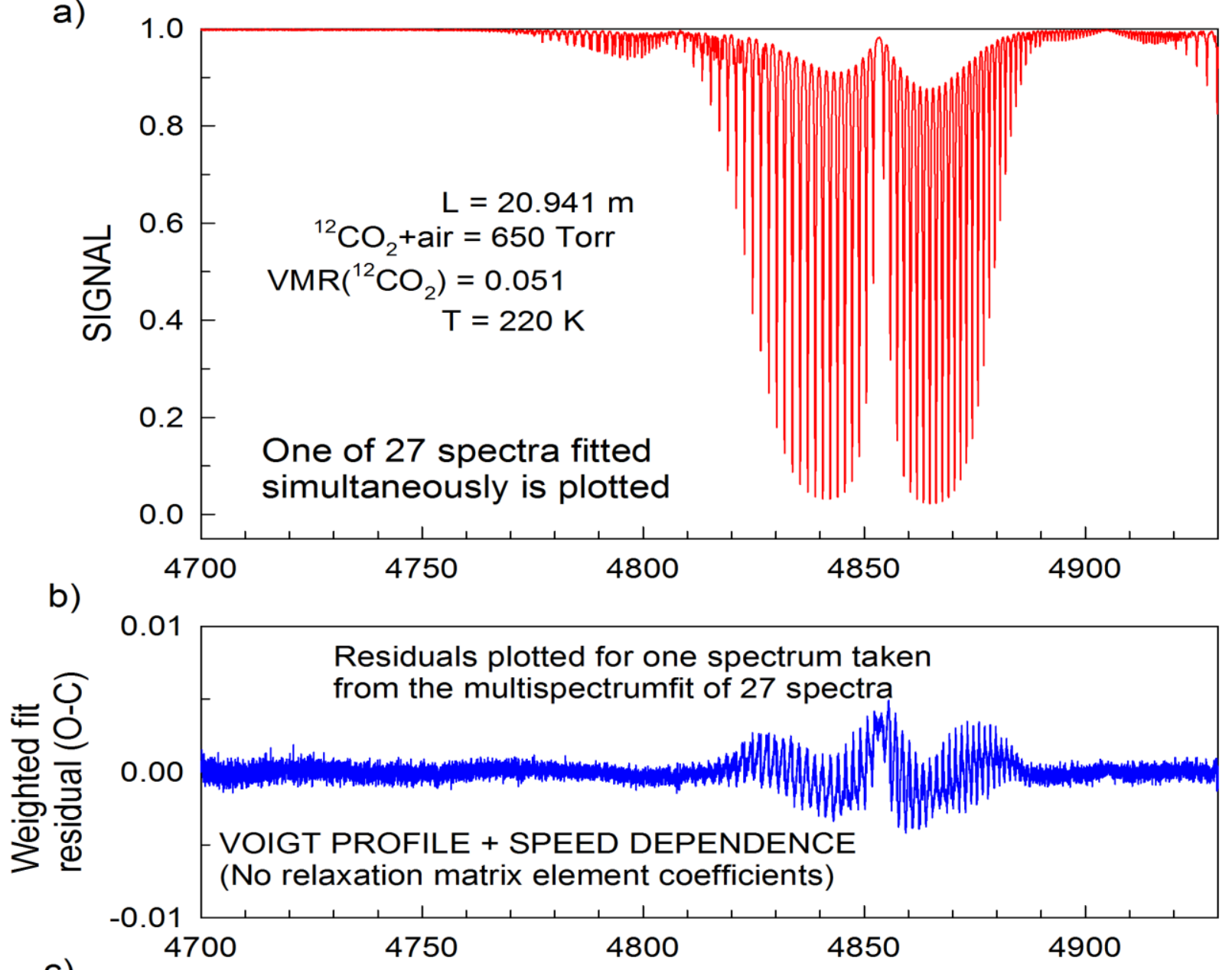

c)

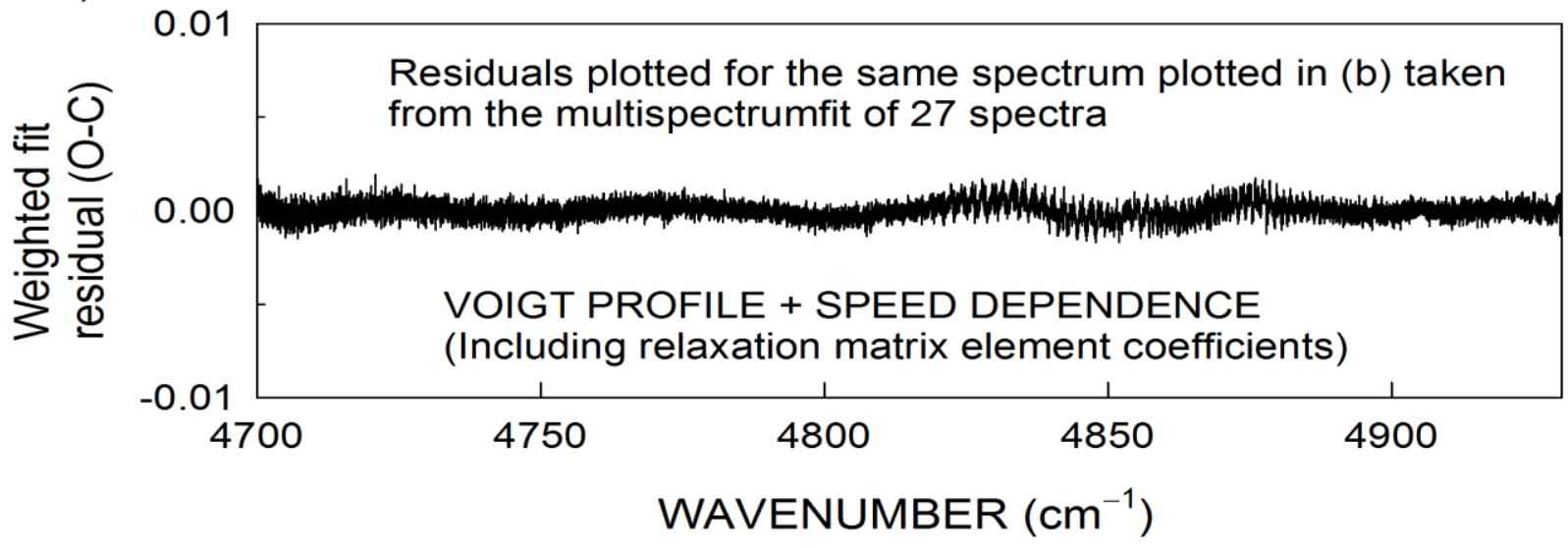

Fig.11 


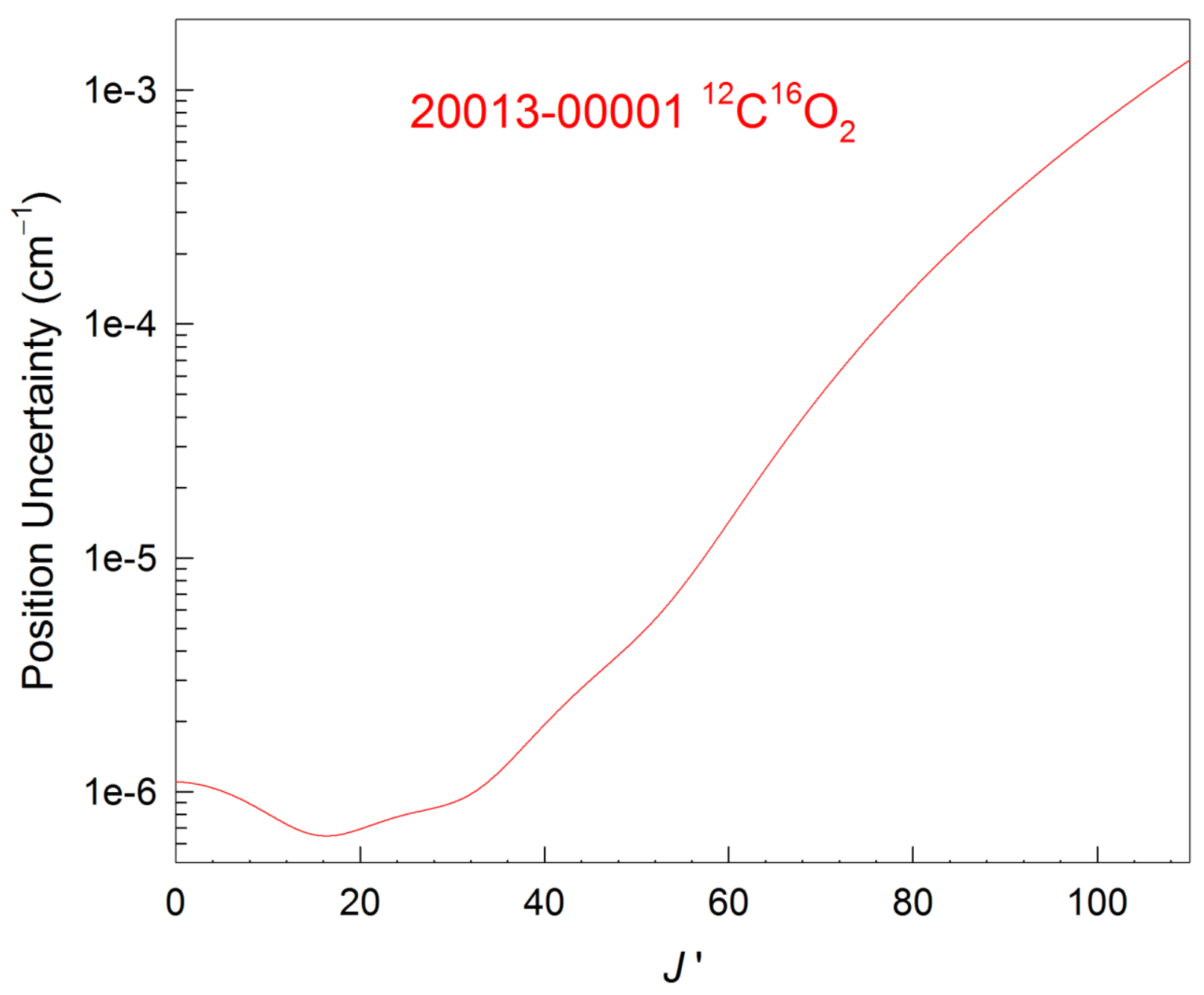

Fig. 12

Figure 


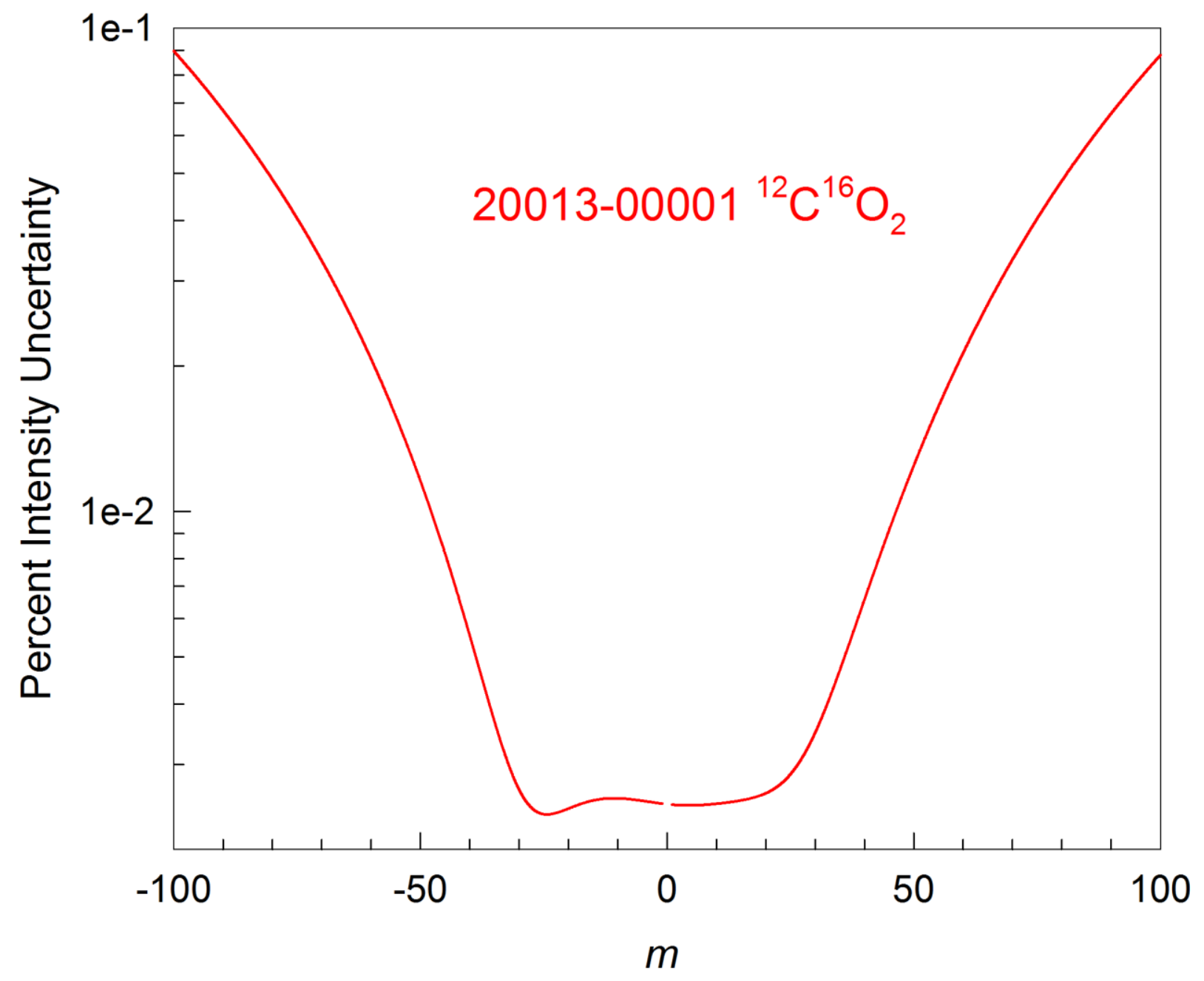

Fig. 13 


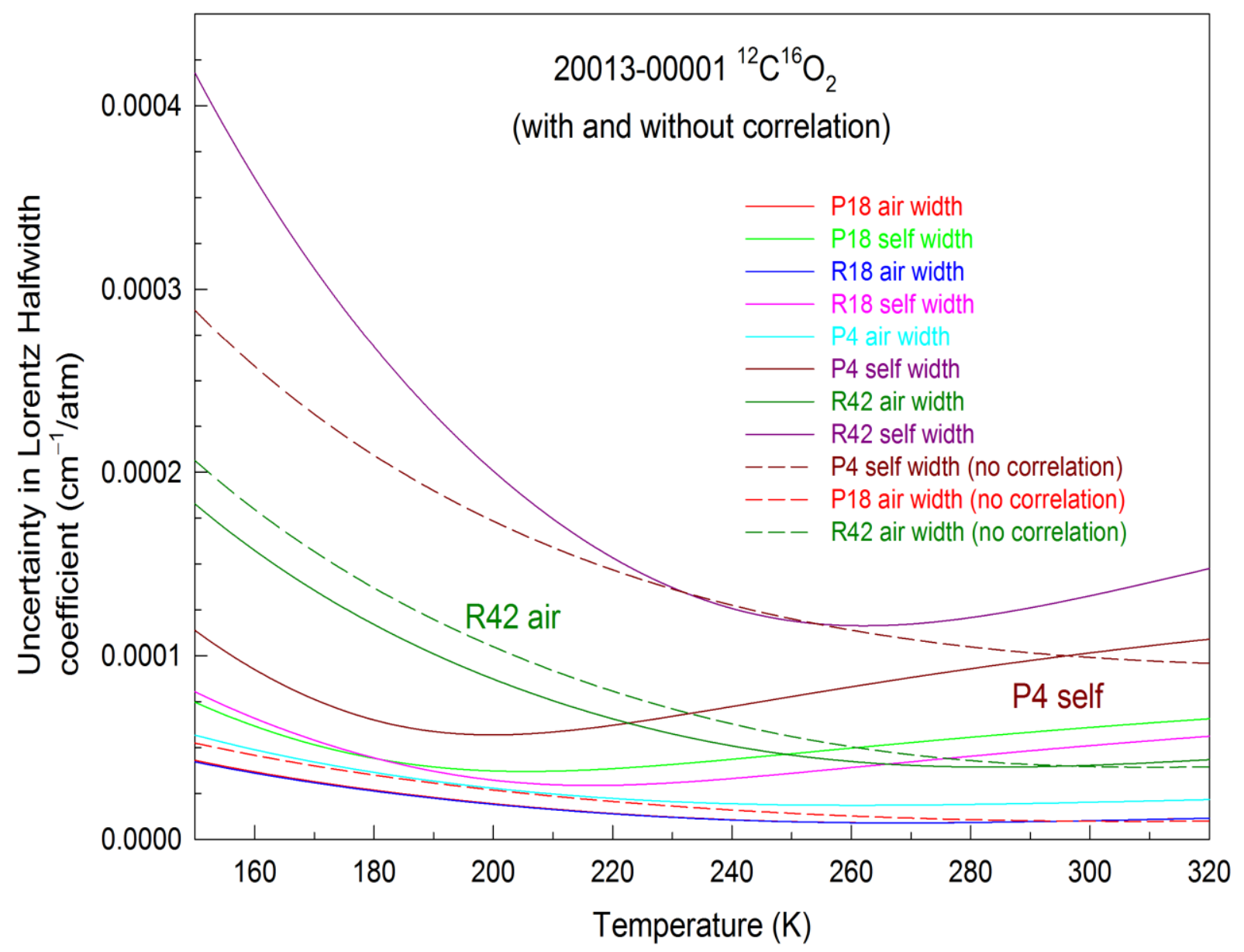

Fig. 14 


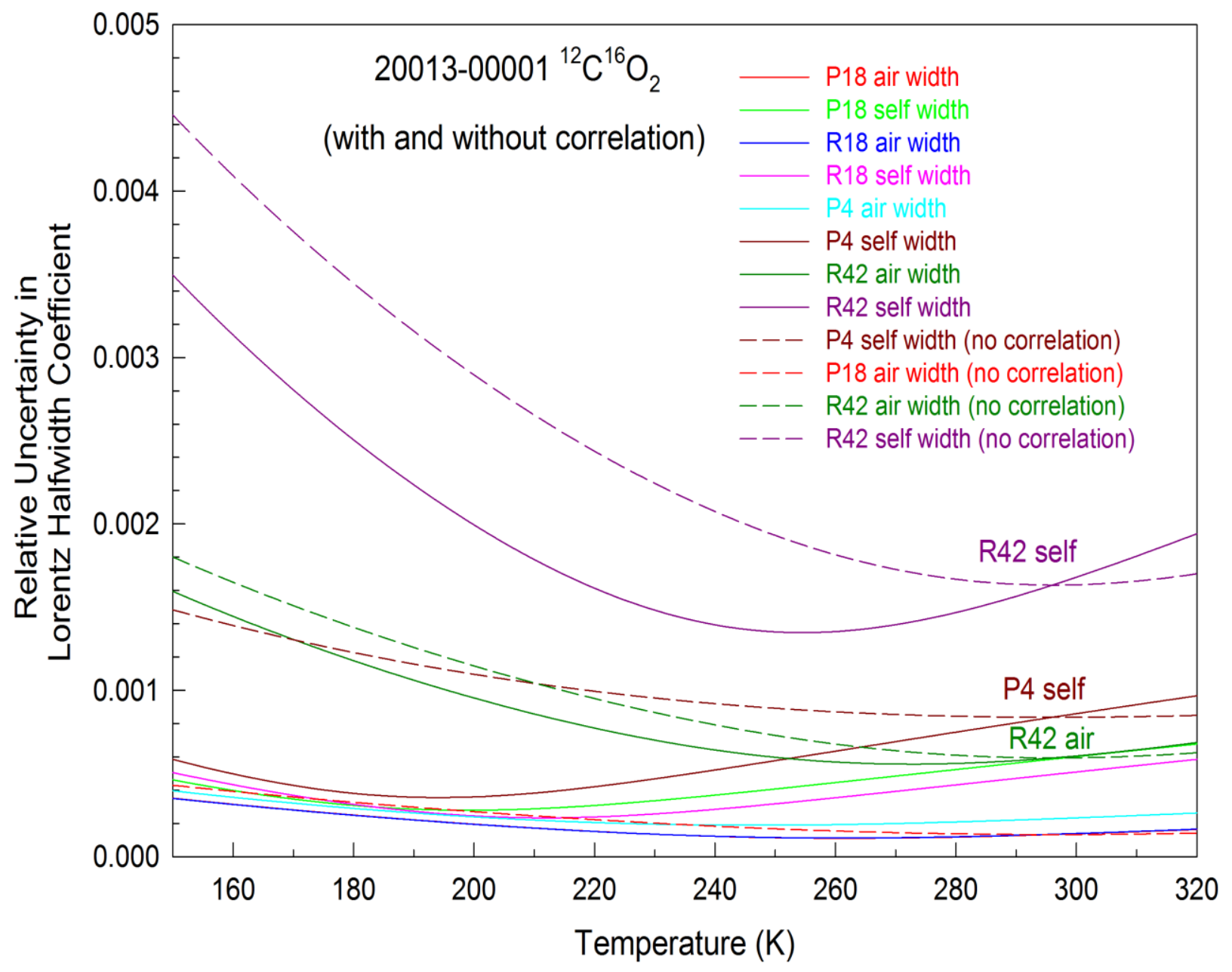

Fig. 15 


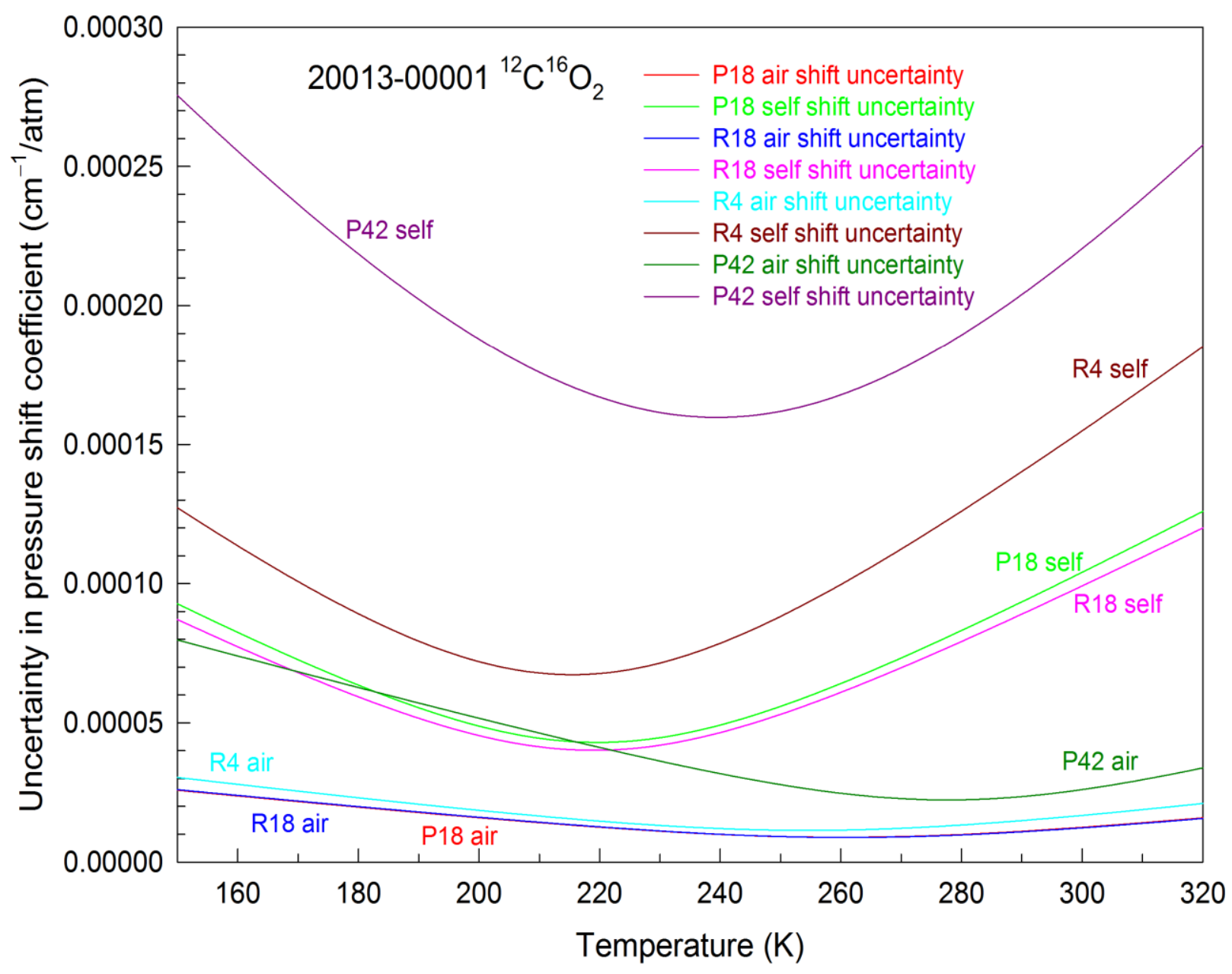

Fig. 16 


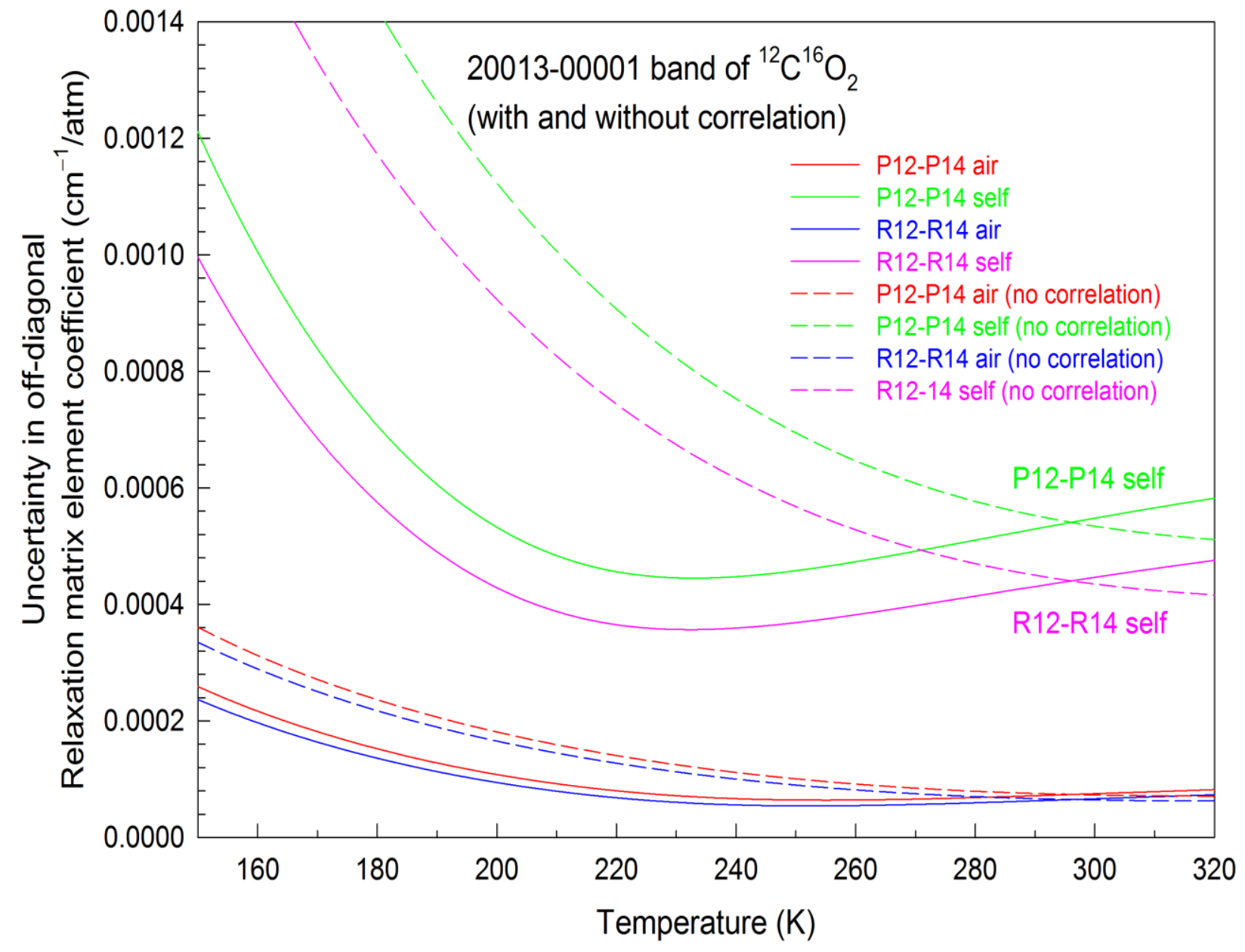

Fig. 17 

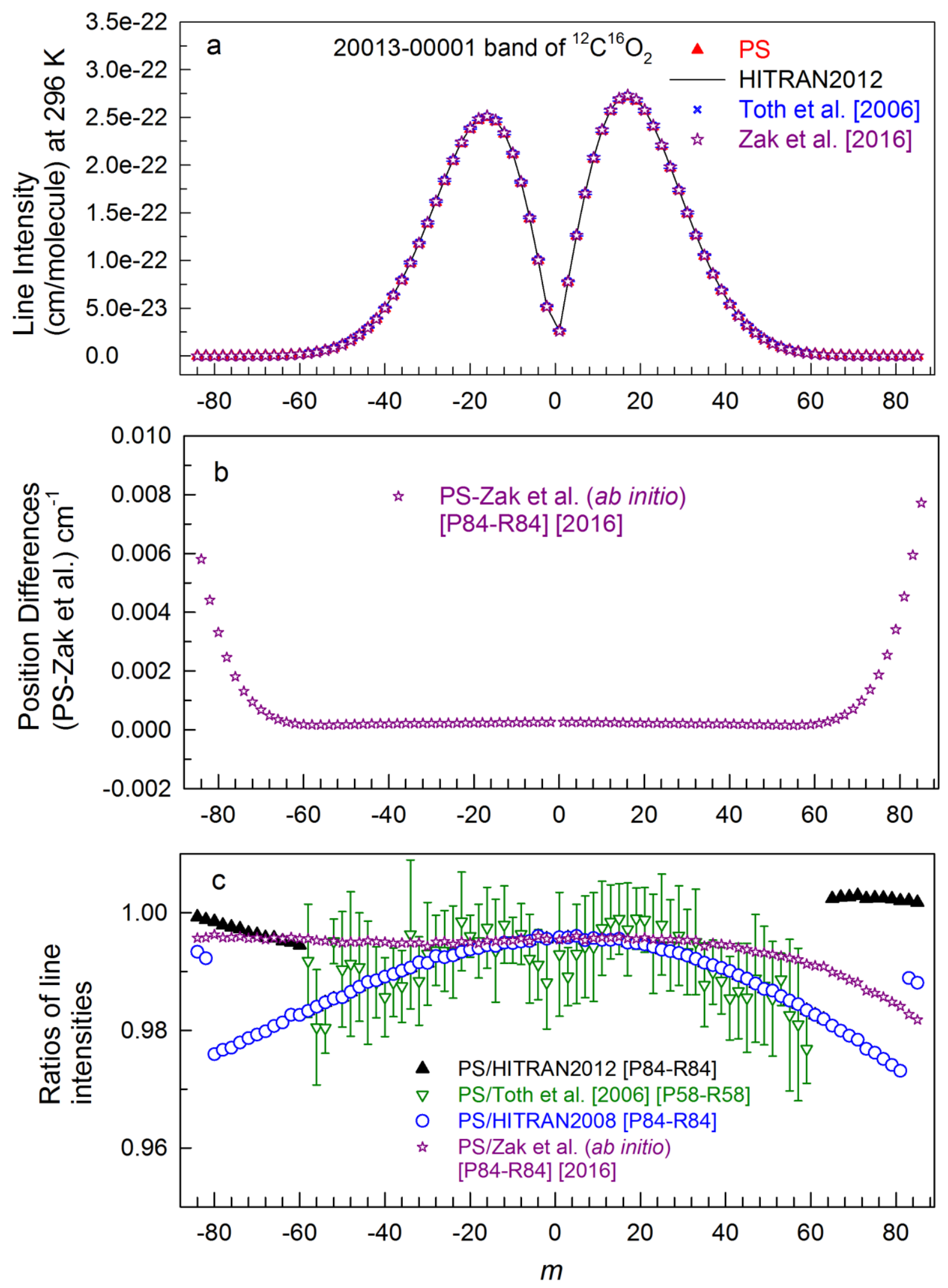
a)

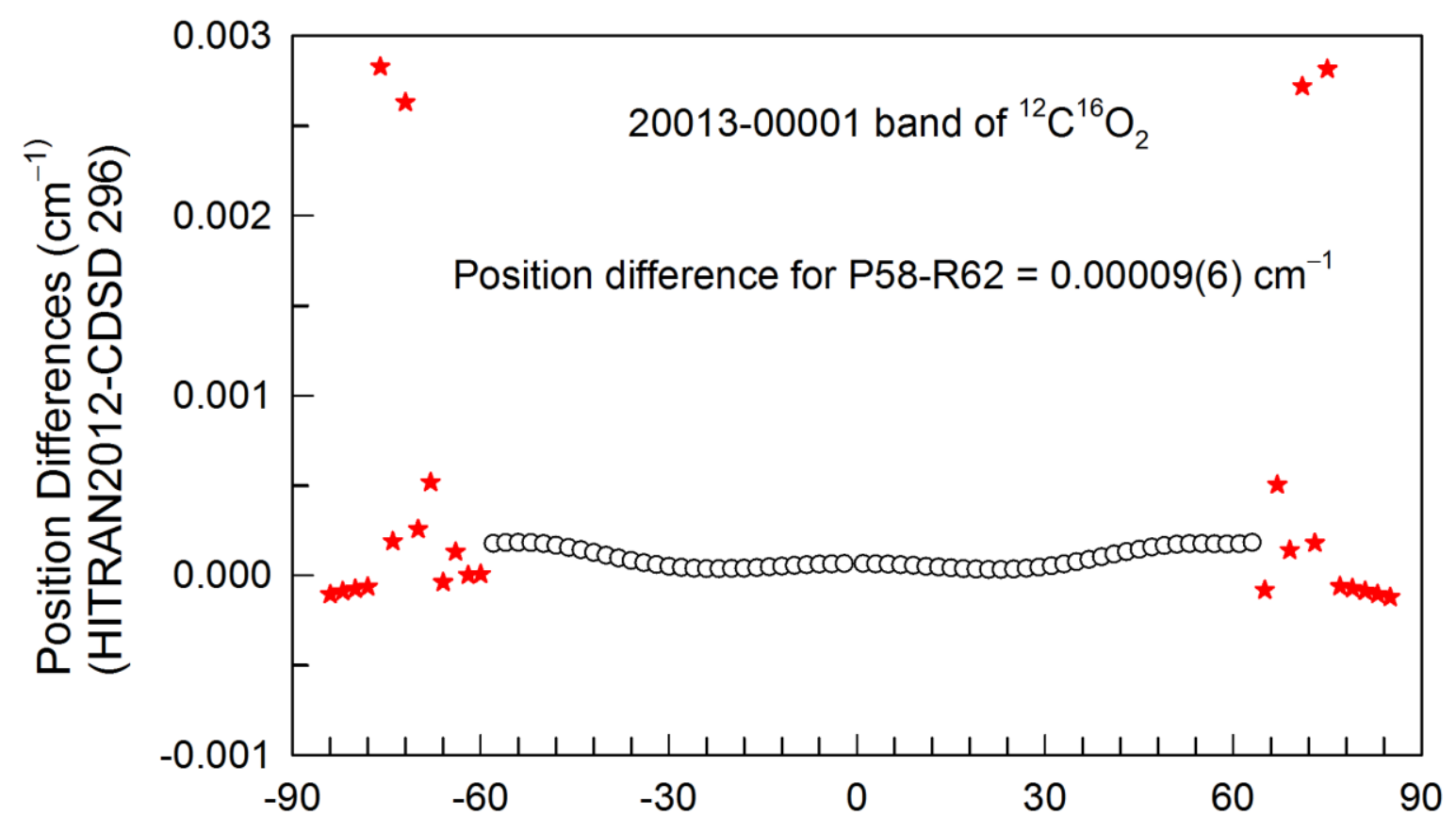

b)

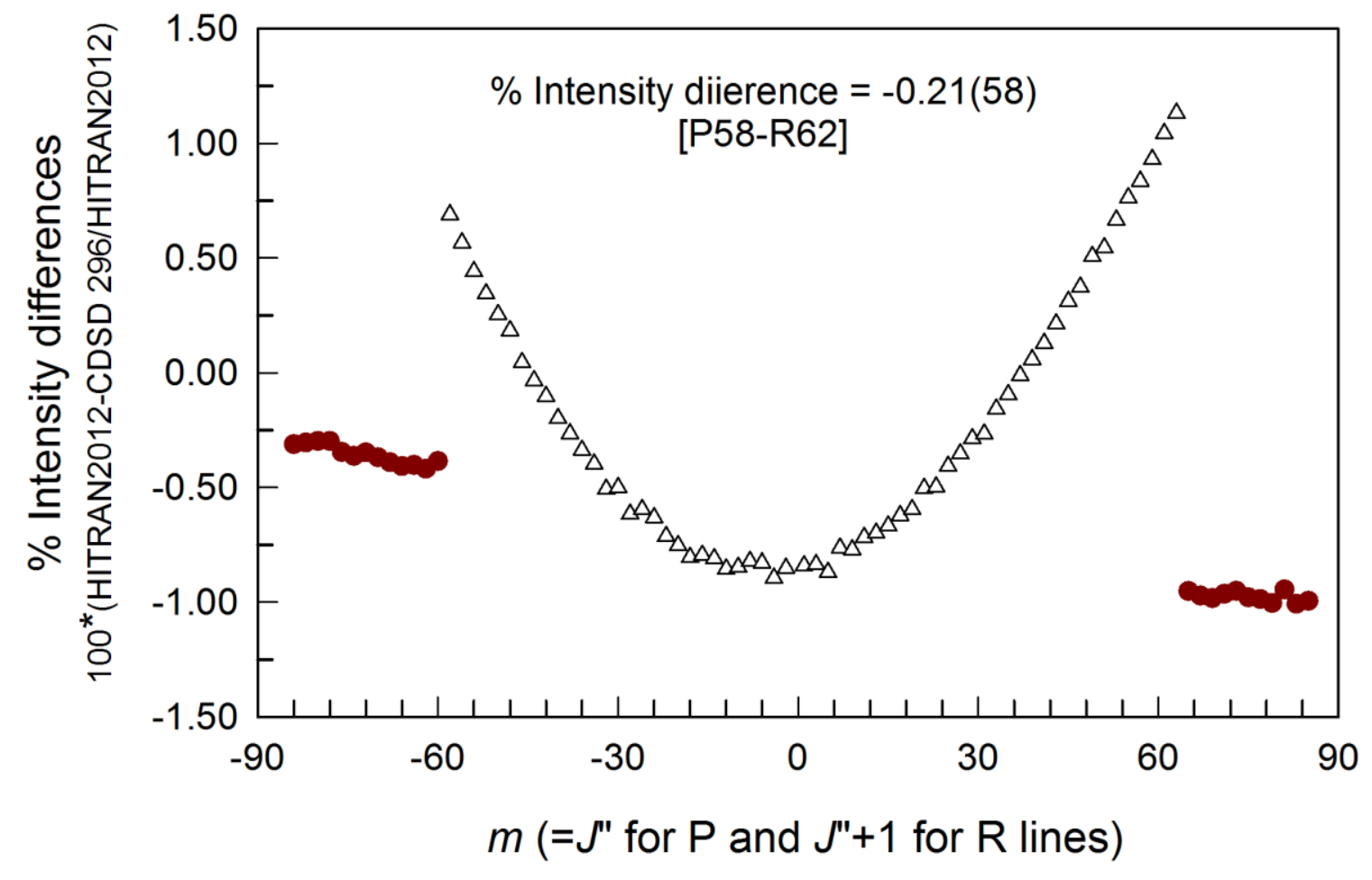

Fig. 19 
a)

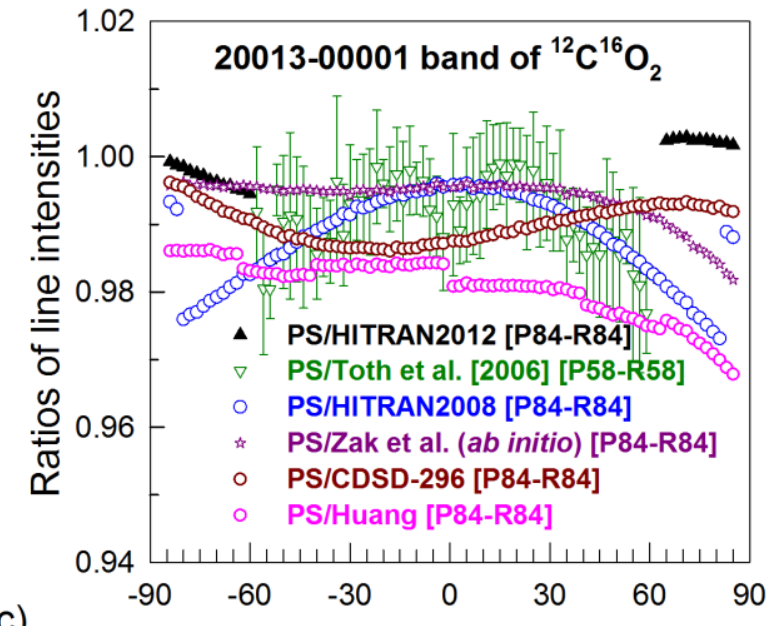

c)

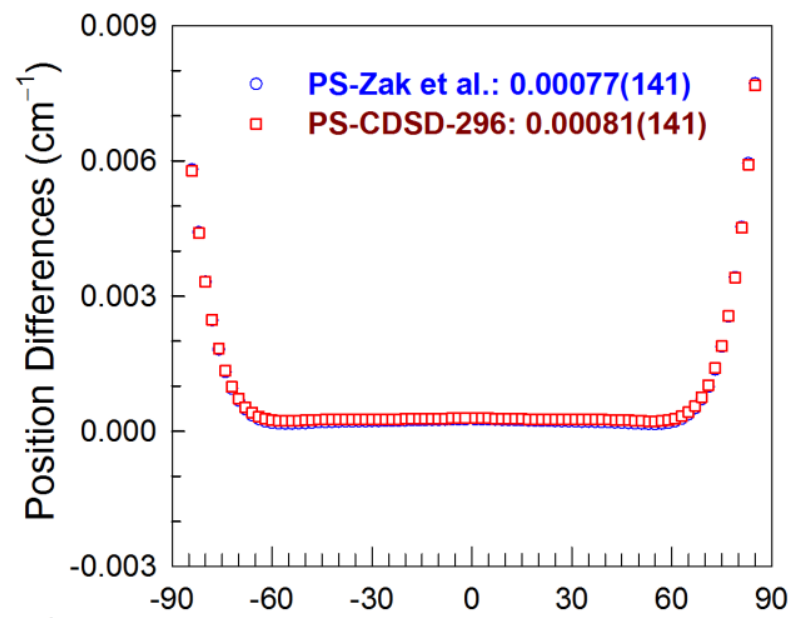

e)

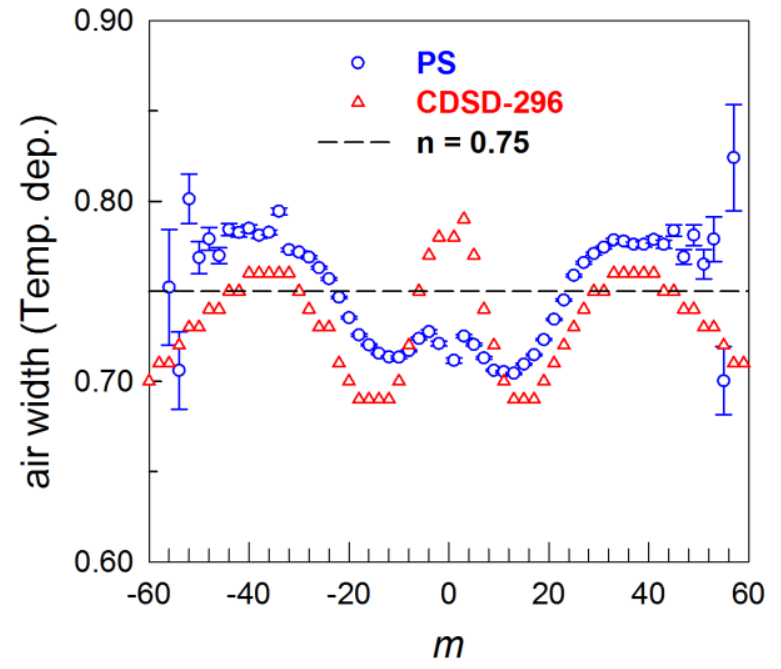

b)

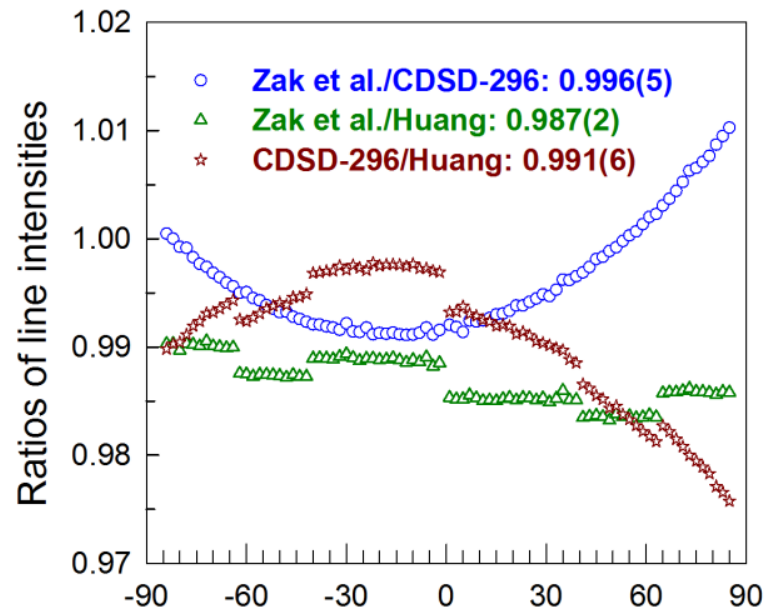

d)

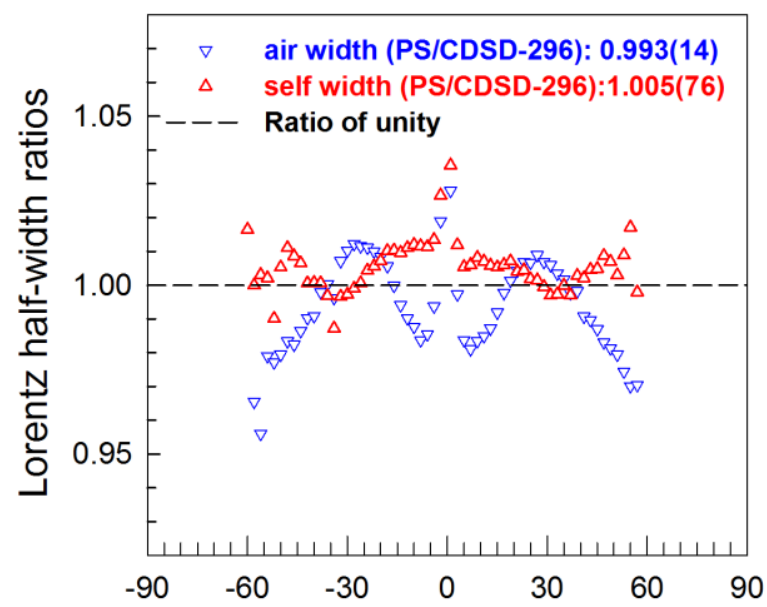

f)

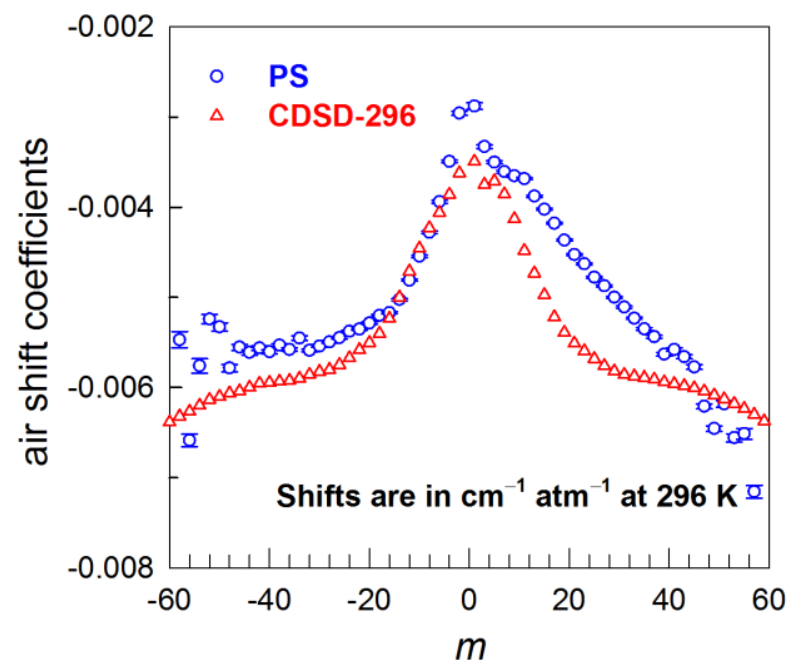

Fig. 20 
Table 1

Summary of line shape measurements ${ }^{\mathrm{a}}$ reported for $\mathrm{CO}_{2}$ bands near $2 \mu \mathrm{m}$.

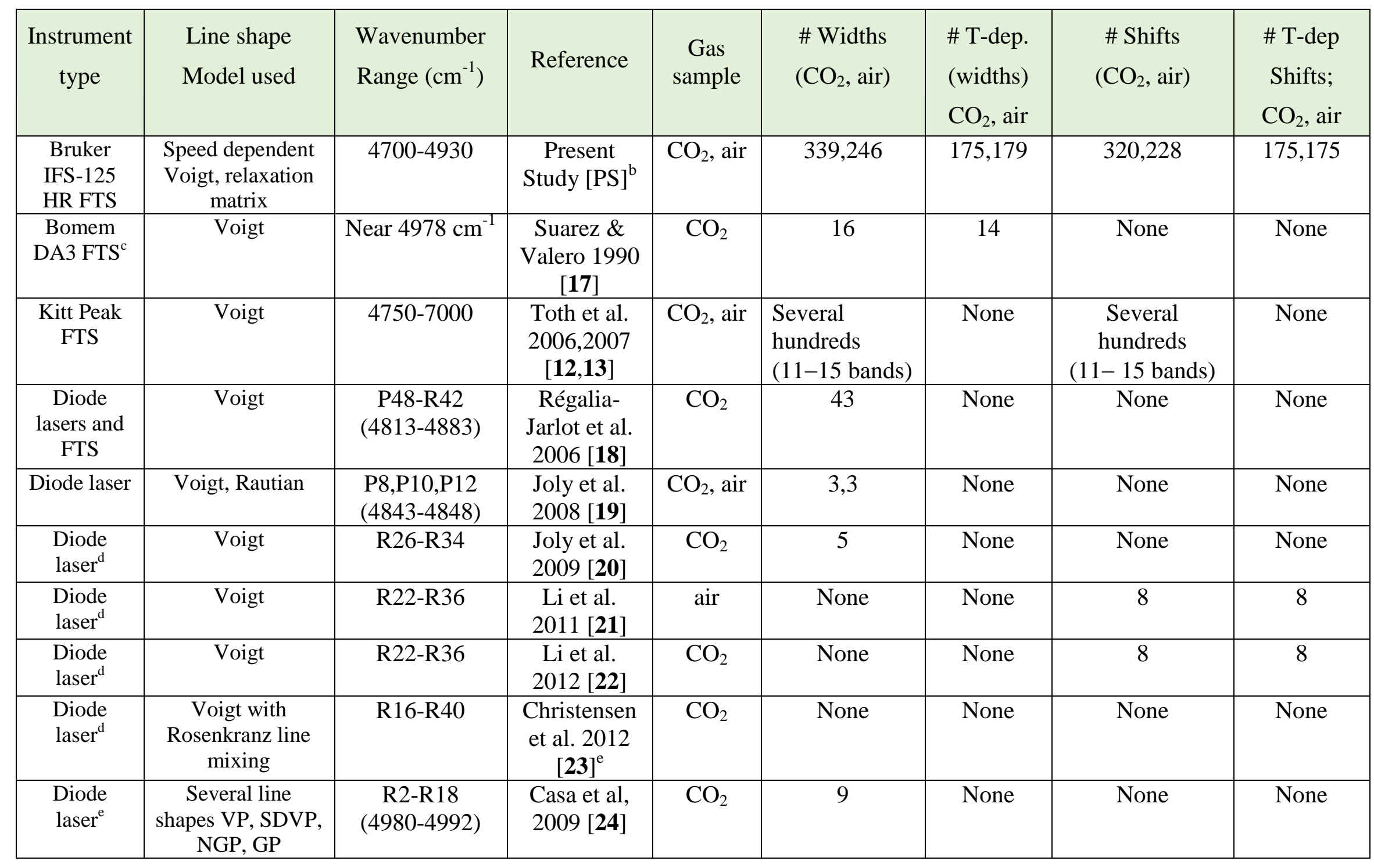


${ }^{a}$ This table lists examples of recent line shape measurements performed using different experimental techniques and analysis methods. For further details, see the original publications. Measurements that involve only line positions and intensities are not included in this table, though they are referenced in the text.

${ }^{\mathrm{b}}$ Line mixing for $\mathrm{CO}_{2}$-air and $\mathrm{CO}_{2}-\mathrm{CO}_{2}$ via off-diagonal relaxation matrix elements formalism are also obtained for a number of transition pairs $(\sim 50)$ for the $20013 \leftarrow 00001$ band of ${ }^{12} \mathrm{C}^{16} \mathrm{O}_{2}$

${ }^{\mathrm{c}} 20012 \leftarrow 00001$ band of ${ }^{12} \mathrm{C}^{16} \mathrm{O}_{2}$.

${ }^{\mathrm{d}}$ Wavenumber coverage is not specified in these papers.

${ }^{\mathrm{e}}$ Rosenkranz line mixing for $\mathrm{CO}_{2}$-air for 13 lines. 
Table 2

Experimental setups and physical conditions of analyzed $\mathrm{CO}_{2}$ spectra.

\begin{tabular}{|c|c|c|}
\hline Parameters & Kitt Peak FTS & $\begin{array}{c}\text { JPL Bruker IFS-125HR } \\
\text { FTS }\end{array}$ \\
\hline Light Source & Quartz halogen Lamp & Tungsten Lamp \\
\hline Bean Splitter & $\mathrm{CaF}_{2}$ & $\mathrm{CaF}_{2}$ \\
\hline Detector & Two matched InSb & $\mathrm{InSb}$ \\
\hline $\begin{array}{c}\text { Focal length of the } \\
\text { collimating lens (mm) }\end{array}$ & 2260 & 418 \\
\hline $\begin{array}{l}\text { Source aperture diameter } \\
\qquad(\mathrm{mm})\end{array}$ & 8 & 1.3 \\
\hline Filter band pass $\left(\mathrm{cm}^{-1}\right)$ & $3000-9000$ & $4200-6400$ \\
\hline Resolution $\left(\mathrm{cm}^{-1}\right)$ Unapodized & $\sim 0.01$ & $0.004-0.011$ \\
\hline $\begin{array}{c}\text { Maximum Optical Path } \\
\text { Difference }(\mathrm{cm})\end{array}$ & 50 & $45-113$ \\
\hline Sample Pressure (Torr) & $10-252$ & $1.1-594$ \\
\hline $\begin{array}{c}\text { Total pressures of } \mathrm{CO}_{2}+\text { air } \\
\text { (Torr) }\end{array}$ & None & $200-711$ \\
\hline Volume mixing ratio & 1.0 & $0.014-.203$ \\
\hline Temperature $(\mathrm{K})$ & $292-297$ & $170-296$ \\
\hline Cell path length (m) & $0.347,2.46,49.0$ & $\begin{array}{l}0.2038,13.09 \\
29.30,20.941 \\
\end{array}$ \\
\hline Scanning time $(\mathrm{h})$ & 1.3 & $5-8$ \\
\hline Signal-to-noise & $800-1000$ & $>2000$ \\
\hline Gas Samples & ${ }^{12} \mathrm{CO}_{2}{ }^{\mathrm{b}},{ }^{13} \mathrm{CO}_{2}{ }^{\mathrm{c}}$ & ${ }^{12}$ C-enriced $\mathrm{CO}_{2}{ }^{\mathrm{d}}$ \\
\hline Calibration standards $^{\mathrm{a}}$ & $\mathrm{CO}, \mathrm{C}_{2} \mathrm{H}_{2}$ & $\mathrm{HCl}, \mathrm{H}_{2} \mathrm{O}$ \\
\hline
\end{tabular}

${ }^{a}$ Calibration of wavenumber scales of spectra was achieved using $\mathrm{CO} / \mathrm{C}_{2} \mathrm{H}_{2} / \mathrm{HCl}$, as appropriate (See the text for details).

${ }^{\mathrm{b}} \mathrm{CO}_{2}$ sample with natural isotopologue abundances was used.

${ }^{\mathrm{c}} 99 \%{ }^{13} \mathrm{C}$-enriched $\mathrm{CO}_{2}$ was used.

${ }^{\mathrm{d}} 99.98 \%{ }^{12} \mathrm{C}$-enriched $\mathrm{CO}_{2}$ sample was used with the $29.3 \mathrm{~m}$ cell while a $99.99 \%{ }^{12} \mathrm{C}$-enriched $\mathrm{CO}_{2}$ sample was used for spectra obtained with the 0.2038 and $20.941 \mathrm{~m}$ cells. 
Table 3

Summary of experimental conditions of spectra analyzed

\begin{tabular}{|c|c|c|c|c|c|}
\hline Serial \# & File name & $\begin{array}{c}\text { Cell } \\
\text { length } \\
(\mathrm{m})^{\mathrm{e}}\end{array}$ & $\begin{array}{c}\text { Total } \\
\text { Pressure } \\
\text { (Torr) }^{\mathrm{f}}\end{array}$ & $\begin{array}{c}\text { Gas } \\
\text { temperature } \\
(\mathrm{K})^{\mathrm{g}}\end{array}$ & $\begin{array}{c}\text { Volume } \\
\text { mixing ratio } \\
\text { of } \mathrm{CO}_{2} \\
\end{array}$ \\
\hline \multicolumn{6}{|c|}{ Pure gas spectra } \\
\hline 1 & $4600 \times 714$. bus $^{\mathrm{a}}$ & 49.0 & 80.00 & 297.40 & 1.0 \\
\hline 2 & $4600 \times 23671$. urs $^{b}$ & 13.09 & 1.108 & 293.80 & 1.0 \\
\hline 3 & $4600 \times 1688 . u^{a} s^{a}$ & 2.46 & 10.005 & 292.38 & 1.0 \\
\hline 4 & $4600 \times 1690 . u^{a} s^{a}$ & 2.46 & 252.01 & 293.37 & 1.0 \\
\hline 5 & 4600x1705.ars ${ }^{\mathrm{c}}$ & 2.46 & 10.04 & 296.61 & 1.0 \\
\hline 6 & 4600x 1677.ars ${ }^{c}$ & 0.347 & 146.56 & 293.54 & 1.0 \\
\hline 7 & $4600 \times 2526$. urs $^{b}$ & 0.2038 & 594.00 & 240.00 & 1.0 \\
\hline 8 & $4600 \times 2529 . \mathrm{urs}^{\mathrm{b}}$ & 0.2038 & 403.60 & 240.00 & 1.0 \\
\hline 9 & 4600x3805.ars ${ }^{b}$ & 20.941 & 0.304 & 220.20 & 1.0 \\
\hline 10 & $4600 \times 2519$. urs $^{b}$ & 0.2038 & 80.00 & 181.65 & 1.0 \\
\hline 11 & $4600 \times 2522$. urs $^{b}$ & 0.2038 & 173.10 & 181.15 & 1.0 \\
\hline 12 & 4600x3831.ars ${ }^{b}$ & 20.941 & 29.70 & 170.30 & 1.0 \\
\hline \multicolumn{6}{|c|}{ Air broadened $\mathrm{CO}_{2}$ spectra } \\
\hline 13 & $4600 \times 2507$. urs $^{\mathrm{d}}$ & 29.30 & 598.90 & 294.10 & 0.014495 \\
\hline 14 & $4600 \times 2508$. urs $^{\mathrm{d}}$ & 29.30 & 401.60 & 294.80 & 0.014464 \\
\hline 15 & $4600 \times 2509$. urs $^{\mathrm{d}}$ & 29.30 & 207.30 & 293.75 & 0.014530 \\
\hline 16 & $4600 \times 2514$. urs $^{\mathrm{d}}$ & 29.30 & 787.71 & 295.70 & 0.05060 \\
\hline 17 & $4600 \times 2515$. urs $^{\mathrm{d}}$ & 29.30 & 599.80 & 296.10 & 0.05058 \\
\hline 18 & 4600x3800.ars & 20.941 & 711.68 & 249.90 & 0.04402 \\
\hline 19 & 4600x3802.ars & 20.941 & 499.00 & 249.90 & 0.04432 \\
\hline 20 & 4600x2527.urs & 0.2038 & 605.90 & 240.00 & 0.1940 \\
\hline 21 & 4600x3809.ars & 20.941 & 649.73 & 220.20 & 0.0509 \\
\hline 22 & 4600x3811.ars & 20.941 & 502.02 & 220.20 & 0.0510 \\
\hline 23 & 4600x3819.ars & 20.941 & 301.25 & 195.00 & 0.03086 \\
\hline 24 & 4600x2523.urs & 0.2038 & 610.34 & 180.00 & 0.2028 \\
\hline 25 & 4600x2520.urs & 0.2038 & 357.15 & 180.00 & 0.1930 \\
\hline 26 & 4600x3827.ars & 20.941 & 400.43 & 169.80 & 0.02985 \\
\hline 27 & 4600x3829.ars & 20.941 & 200.41 & 170.10 & 0.03000 \\
\hline
\end{tabular}

Notes:

$1 \mathrm{~atm}=101.3 \mathrm{kPa}=760$ Torr.

${ }^{\mathrm{a}}$ Natural $\mathrm{CO}_{2}$ sample.

${ }^{\mathrm{b}} 99.99 \%{ }^{12} \mathrm{C}$-enriched $\mathrm{CO}_{2}$.

${ }^{\mathrm{c}} 99 \%{ }^{13} \mathrm{C}$-enriched $\mathrm{CO}_{2}$.

${ }^{\mathrm{d}} 99.98 \%{ }^{12} \mathrm{C}$-enriched $\mathrm{CO}_{2}$ was used with the $29.30 \mathrm{~m}$ cell and a $99.99 \%{ }^{12} \mathrm{C}$-enriched sample was used for the data taken with the 0.2038 and $20.941 \mathrm{~m}$ cells.

${ }^{\mathrm{e}}$ Cell lengths are known with uncertainties of 0.01-0.3\% depending on the cell (see the text for details).

${ }^{\mathrm{f}}$ Gas pressures are measured with uncertainties $\pm 0.05 \%$ of full-scale pressure readings.

${ }^{\mathrm{g}}$ Cell (gas sample) temperatures are measured with uncertainties of $\pm 0.02-0.2 \mathrm{~K}$. 
Table 4

Major bands included in the $2 \mu \mathrm{m}$ region $^{\mathrm{a}}$ of the fitted interval.

\begin{tabular}{|c|c|c|c|c|c|c|}
\hline $\begin{array}{l}\# \text { of } \\
\text { band }\end{array}$ & $\mathrm{V}^{\prime}$ & $V^{\prime \prime}$ & $\begin{array}{c}\text { P branch } \\
\text { Range in } J\end{array}$ & $\begin{array}{l}\text { Q branch } \\
\text { Range in } J\end{array}$ & $\begin{array}{l}\text { R branch } \\
\text { Range in } J\end{array}$ & Iso. \\
\hline 1 & 20013 & 00001 & $2-84$ & & $0-84$ & ${ }^{12} \mathrm{C}^{16} \mathrm{O}_{2}$ \\
\hline 2 & 20012 & 00001 & $2-72$ & & $0-72$ & ${ }^{13} \mathrm{C}^{16} \mathrm{O}_{2}$ \\
\hline 3 & 20013 & 00001 & $2-60$ & & $0-64$ & ${ }^{13} \mathrm{C}^{16} \mathrm{O}_{2}$ \\
\hline 4 & 40002 & 01101 & $3-55$ & $2-20$ & $1-55$ & ${ }^{12} \mathrm{C}^{16} \mathrm{O}_{2}{ }^{\mathrm{a}}$ \\
\hline 5 & 21113 & $01101 e, f$ & $2-73$ & $1-38$ & $1-72$ & ${ }^{12} \mathrm{C}^{16} \mathrm{O}_{2}{ }^{\mathrm{a}}$ \\
\hline 6 & 20013 & 00001 & $1-64$ & & $0-64$ & ${ }^{12} \mathrm{C}^{16} \mathrm{O}^{18} \mathrm{O}$ \\
\hline 7 & 30014 & 10002 & $2-64$ & & $0-64$ & ${ }^{12} \mathrm{C}^{16} \mathrm{O}_{2}$ \\
\hline 8 & 20012 & 00001 & $48-88$ & & & ${ }^{12} \mathrm{C}^{16} \mathrm{O}_{2}$ \\
\hline 9 & 21112 & $01101 e, f$ & $36-77$ & & & ${ }^{12} \mathrm{C}^{16} \mathrm{O}_{2}$ \\
\hline 10 & 30013 & 10002 & $12-68$ & & & ${ }^{12} \mathrm{C}^{16} \mathrm{O}_{2}$ \\
\hline 11 & 20012 & 00001 & $1-67$ & & $0-49$ & ${ }^{12} \mathrm{C}^{16} \mathrm{O}^{18} \mathrm{O}$ \\
\hline 12 & 30012 & 10001 & $30-66$ & & & ${ }^{12} \mathrm{C}^{16} \mathrm{O}_{2}$ \\
\hline 13 & 22212 & $02201 e, f$ & $24-64$ & & & ${ }^{12} \mathrm{C}^{16} \mathrm{O}_{2}$ \\
\hline 14 & 30013 & 10001 & $2-60$ & & $0-60$ & ${ }^{12} \mathrm{C}^{16} \mathrm{O}_{2}$ \\
\hline 15 & 22213 & $02201 e, f$ & $3-60$ & $2-28$ & $2-60$ & ${ }^{12} \mathrm{C}^{16} \mathrm{O}_{2}$ \\
\hline 16 & 21112 & $01101 e, f$ & $2-57$ & $1-14$ & $1-58$ & ${ }^{12} \mathrm{C}^{16} \mathrm{O}_{2}$ \\
\hline 17 & 20013 & 00001 & $1-53$ & & $0-53$ & ${ }^{12} \mathrm{C}^{16} \mathrm{O}^{17} \mathrm{O}$ \\
\hline 18 & 20012 & 00001 & $8-58$ & & & ${ }^{12} \mathrm{C}^{16} \mathrm{O}^{17} \mathrm{O}$ \\
\hline 19 & 31113 & $11102 e, f$ & $2-53$ & $1-8$ & $1-2$ & ${ }^{12} \mathrm{C}^{16} \mathrm{O}_{2}$ \\
\hline 20 & 12212 & 00001 & $12-70$ & & $10-64$ & ${ }^{12} \mathrm{C}^{16} \mathrm{O}_{2}{ }^{b}$ \\
\hline 21 & 23301 & 00001 & $\begin{array}{c}\text { No line } \\
\text { observed }\end{array}$ & & & ${ }^{12} \mathrm{C}^{16} \mathrm{O}_{2}{ }^{b}$ \\
\hline
\end{tabular}

${ }^{a}$ Some of the bands are fragmentary and only the P-branch transitions appear in the fitted interval. Line parameters for a number of weaker transitions were kept fixed in the least squares solution. $\mathrm{V}^{\prime}$ : Upper vibrational level

$\mathrm{V}^{\prime \prime}$ : Lower vibrational level

The numbers (e.g., 2-84) under $\mathrm{P}, \mathrm{Q}$ and $\mathrm{R}$ branch denote the range in $J^{\prime \prime}$ for observed transitions Iso: Isotopologue $\left({ }^{12} \mathrm{C}^{16} \mathrm{O}_{2},{ }^{13} \mathrm{C}^{16} \mathrm{O}_{2},{ }^{12} \mathrm{C}^{16} \mathrm{O}^{18} \mathrm{O},{ }^{12} \mathrm{C}^{16} \mathrm{O}^{18} \mathrm{O}\right)$.

${ }^{a}$ Bands that are interacting.

b $23301 \leftarrow 00001$ is a dark state and no lines were observable in our spectral data; however, it interacts with the band $12212 \leftarrow 00001$.

Transitions from a total of 42 vibrational bands $\left(24,9,2,3\right.$ and 4 bands belonging to ${ }^{12} \mathrm{C}^{16} \mathrm{O}_{2}$, ${ }^{13} \mathrm{C}^{16} \mathrm{O}_{2},{ }^{12} \mathrm{C}^{16} \mathrm{O}^{18} \mathrm{O},{ }^{12} \mathrm{C}^{16} \mathrm{O}^{17} \mathrm{O}$ and ${ }^{13} \mathrm{C}^{16} \mathrm{O}^{18} \mathrm{O}$ ) were considered in the fitted region (several of which are very weak and their parameters were held fixed in the fittings). see Supplemental file \#3. 


\section{Table 5}

Measured line parameters and number of $\mathrm{CO}_{2}$ measurements.

\begin{tabular}{|c|c|c|c|}
\hline Parameters & $\begin{array}{c}\text { Number of } \\
\text { measurements }\end{array}$ & Parameters & $\begin{array}{c}\text { Number of } \\
\text { measurements }\end{array}$ \\
\hline Position $(v)^{\mathrm{a}}$ & $\begin{array}{c}533 \text { constrained } \\
(1464 \text { total })\end{array}$ & Intensity $(S)^{\mathrm{b}}$ & $\begin{array}{l}625 \text { constrained } \\
(1514 \text { total })\end{array}$ \\
\hline $\begin{array}{c}\text { Air-broadened width } \\
\text { Coefficient (air-Width) }\end{array}$ & 246 & $\begin{array}{c}\text { Self-broadened width } \\
\text { Coefficient (self-Width) }^{\mathrm{c}}\end{array}$ & 339 \\
\hline $\begin{array}{l}\text { Temperature dependence of } \\
\text { air-broadened width } \\
\text { coefficient }^{\mathrm{d}}\left(n_{1}\right)\end{array}$ & 179 & $\begin{array}{l}\text { Temperature dependence of } \\
\text { self-broadened width } \\
\text { coefficient }^{\mathrm{d}}\left(n_{2}\right)\end{array}$ & 175 \\
\hline 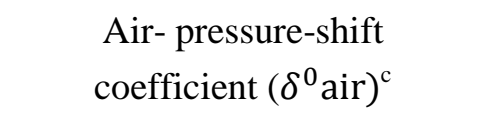 & 228 & $\begin{array}{l}\text { Self- pressure-shift } \\
\text { coefficient }\left(\delta^{0} \text { self }\right)^{c}\end{array}$ & 320 \\
\hline $\begin{array}{l}\text { Temperature dependence of } \\
\text { air- pressure-shift coefficient } \\
\left(\delta^{\prime}\right)\end{array}$ & 175 & $\begin{array}{c}\text { Temperature dependence of } \\
\text { self- pressure-shift } \\
\text { coefficient }^{\mathrm{e}}\left(\delta^{\prime}\right)\end{array}$ & 175 \\
\hline $\begin{array}{l}\text { Off-diagonal relaxation matrix } \\
\text { elements for } \mathrm{CO}_{2}+\text { air mixing } \\
\qquad\left(W_{\mathrm{ij}}\right)^{\mathrm{c}}\end{array}$ & 54 & $\begin{array}{l}\text { Off-diagonal relaxation } \\
\text { matrix elements for } \\
\mathrm{CO}_{2}+\mathrm{CO}_{2} \text { mixing }\left(W_{\mathrm{ij}}\right)^{\mathrm{c}}\end{array}$ & 49 \\
\hline $\begin{array}{l}\text { Temp. dependence of off- } \\
\text { diagonal relaxation matrix } \\
\text { element coefficients for } \\
\mathrm{CO}_{2}+\text { airmixing }\end{array}$ & 26 & $\begin{array}{l}\text { Temp. dependence of off- } \\
\text { diagonal relaxation matrix } \\
\text { element coefficients for } \\
\mathrm{CO}_{2}+\mathrm{CO}_{2} \text { mixing }\end{array}$ & 25 \\
\hline Speed dependence & 112 & Auxiliary parameters ${ }^{\mathrm{f}}$ & 98 \\
\hline
\end{tabular}

${ }^{a}$ The number of measured positions (in $\mathrm{cm}^{-1}$ ) is less than the measured line intensities; positions of many lines were held fixed due to their weakness, blends and proximity to stronger lines. First row: Number of measured positions and intensities with constraints are given first, followed by all measured positions and intensities at the bottom in parentheses (this number also includes the number given above).

${ }^{\mathrm{b}}$ Line intensity in $\mathrm{cm} /$ molecule at $296 \mathrm{~K}$.

${ }^{\mathrm{c}}$ Lorentz half-width and pressure-shift coefficients and the off-diagonal relaxation matrix element coefficients in $\mathrm{cm}^{-1} \mathrm{~atm}^{-1}$ at $296 \mathrm{~K}$.

${ }^{\mathrm{d}}$ Temperature dependence exponents of half-width coefficients and the off-diagonal relaxation matrix element coefficients, and the speed dependence parameters have no units.

${ }^{\mathrm{e}}$ Temperature dependence of pressure-shift coefficients in $\mathrm{cm}^{-1} \mathrm{~atm}^{-1} \mathrm{~K}^{-1}$.

${ }^{\mathrm{f}}$ Examples include ro-vibrational constants, vibrational band strengths $\left(S_{\mathrm{v}}\right)$ (see text for details). 
Table 6(a)

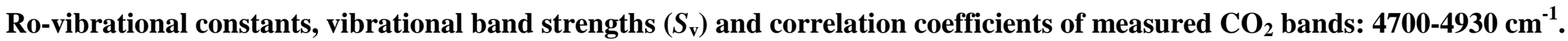

\begin{tabular}{|c|c|c|c|c|c|c|c|c|c|c|c|c|c|c|c|}
\hline \multirow[t]{2}{*}{ Band/iso. } & \multicolumn{8}{|c|}{ UpperState Rotational Constants in $\mathrm{cm}^{-1}$} & \multicolumn{7}{|c|}{ Intensity Constants } \\
\hline & \multicolumn{2}{|c|}{$G^{\prime}-G^{\prime \prime}$} & \multicolumn{3}{|c|}{$B^{\prime}$} & \multicolumn{2}{|c|}{$D^{\prime} \times 10^{7}$} & $H^{\prime} \times 10^{12}$ & \multicolumn{2}{|c|}{$S_{V} \times 10^{23}$} & \multicolumn{2}{|c|}{$a_{1} \times 10^{4}$} & $a_{2} \times 10^{5}$ & \multicolumn{2}{|c|}{$a_{3} \times 10^{8}$} \\
\hline $20013 \leftarrow 00001(626)$ & \multicolumn{2}{|c|}{$4853.623370(1)$} & \multicolumn{3}{|c|}{$0.3881977360(44)$} & \multicolumn{2}{|c|}{$1.817979(43)$} & $0.5833(10)$ & \multicolumn{2}{|c|}{$688.420(2)$} & \multicolumn{2}{|c|}{$+2.342(6)$} & $+3.0063(16)$ & \multicolumn{2}{|c|}{$-0.371(80)$} \\
\hline $\begin{array}{l}21113 \leftarrow 01101 e(626)^{\mathrm{a}} \\
21113 \leftarrow 01101 f\end{array}$ & \multicolumn{2}{|c|}{$4807.694373(12)$} & \multicolumn{3}{|c|}{$\begin{array}{l}0.388134609(160) \\
0.389436575(53)\end{array}$} & \multicolumn{2}{|c|}{$\begin{array}{l}1.624471(115) \\
1.759166(57)\end{array}$} & $\begin{array}{l}0.396(24) \\
0.233(15)\end{array}$ & \multicolumn{2}{|c|}{$65.488(6)^{\mathrm{c}}$} & \multicolumn{2}{|c|}{$-0.07(11)$} & $-15.5(10)$ & \multicolumn{2}{|c|}{$+2.85(5)$} \\
\hline $40002 \longleftarrow 01101(626)^{b}$ & \multicolumn{2}{|c|}{$4808.173871(83)$} & \multicolumn{3}{|c|}{$0.39008047(47)$} & \multicolumn{2}{|c|}{$0.76606(57)$} & $1.68(19)$ & \multicolumn{2}{|c|}{$0.00123^{\mathrm{c}}$} & \multicolumn{2}{|c|}{$+641 .(25)$} & $+958 .(51)$ & \multicolumn{2}{|c|}{$-3789 .(280)$} \\
\hline $30014 \leftarrow 10002(626)$ & \multicolumn{2}{|c|}{$4790.571556(57)$} & & $8906302(272$ & & 2.1 & $22(29)$ & $1.647(84)$ & 3.331 & (41) & & $0.69(35)$ & $0.11(3)$ & 0.00 & $3(4)$ \\
\hline $30013 \leftarrow 10002(626)$ & 4942.50875 & $8(112)$ & 0.3 & $671049(36)$ & & 1.7 & $496(307)$ & $1.052(75)$ & 11.229 & & & $9.8(3)$ & $-8.60(5)$ & 0.0 & fixed) \\
\hline $20012 \leftarrow 00001(636)$ & 4887.38460 & $5(8)$ & 0.3 & $6855580(30)$ & & 1.5 & $655(30)$ & $0.566(8)$ & 26.690 & & & $1.841(8)$ & $1.288(2)$ & -1.9 & \\
\hline $20013 \leftarrow 00001(636)$ & 4748.05936 & $7(24)$ & 0.3 & $8854741(116)$ & & 1.8 & $10(14)$ & $10.52(7)$ & 2.7080 & & & $2.76(25)$ & $2.94(8)$ & $-4 .($ & \\
\hline $20013 \leftarrow 00001(628)$ & 4791.25951 & 2(19) & 0.3 & $5760127(111)$ & & 1.5 & $43(15)$ & $0.401(50)$ & 4.8692 & & & $4.32(25)$ & $1.43(8)$ & -9.4 & \\
\hline $20012 \leftarrow 00001(628)$ & 4904.85974 & $3(21)$ & 0.3 & $4844268(107)$ & & 1.1 & 49(13) & $0.846(42)$ & 12.9875 & 58) & & $2.66(14)$ & $-6.78(50)$ & -12.9 & 18) \\
\hline & & & & LowerState & $\mathrm{Co}$ & tants & & & & & & & & & \\
\hline 00001 (fixed) (626) & 0. & & 0.3 & 0218949 & & & 4088 & 1.918 & & & & & & & \\
\hline $\begin{array}{l}01101 e \text { (fixed) (626) } \\
01101 f \text { (fixed) }(626)\end{array}$ & 667.379826 & & & $\begin{array}{l}0639109 \\
1254698\end{array}$ & & & 3930 & $\begin{array}{l}2.967 \\
3.040\end{array}$ & & & & & & & \\
\hline $\begin{array}{l}10001 \text { (fixed) (626) } \\
10002 \text { (fixed) (626) }\end{array}$ & $\begin{array}{l}1388.18409 \\
1285.40811\end{array}$ & & & $\begin{array}{l}0188916 \\
0482292\end{array}$ & & & $\begin{array}{l}94271 \\
15707\end{array}$ & $\begin{array}{l}1.86596 \\
2.33398\end{array}$ & & & & & & & \\
\hline 00001 (fixed) (636) & 0. & & 0.3 & 0237280 & & & 2981 & 1.34 & & & & & & & \\
\hline 00001 (fixed) (628) & 0. & & 0.3 & 8184557 & & & 7385 & 62.60 & & & & & & & \\
\hline Band & & Cor & tior & Coefficients fo & or $\mathbf{U}$ & perS & te Consta & & & Corr & lation & Coefficien & ts for Intensit & Constant & \\
\hline & $G^{\prime}-G^{\prime \prime} \& B^{\prime}$ & $G^{\prime}-G$ & & $G^{\prime}-G^{\prime \prime} \& H^{\prime}$ & & & $B^{\prime} \& H^{\prime}$ & $D^{\prime} \& H^{\prime}$ & $S_{V} \& A_{I}$ & $S_{V} d$ & & $S_{V} \& A_{3}$ & $A_{1} \& A_{2}$ & $A_{1} \& A_{3}$ & $A_{2} \& A_{3}$ \\
\hline $20013 \leftarrow 00001 \quad(626)$ & -0.7798 & -0.62 & & -0.4985 & & 355 & +0.8129 & +0.9478 & -0.0540 & -0.5 & 935 & +0.0900 & +0.1154 & -0.8130 & -0.2405 \\
\hline $30014 \leftarrow 10002 \quad(626)$ & -0.8464 & -0.72 & & -0.6106 & & 576 & +0.8582 & +0.9663 & +0.0560 & -0.7 & 540 & -0.0311 & -0.0382 & -0.8392 & -0.0096 \\
\hline $30013 \leftarrow 10002 \quad(626)$ & -0.9652 & -0.91 & & -0.8350 & & 804 & +0.9242 & +0.9782 & +0.9886 & +0. & 567 & ---- & +0.9878 & ---- & ---- \\
\hline $20012 \leftarrow 00001 \quad(636)$ & -0.7951 & -0.61 & & -0.4689 & & 234 & +0.7806 & +0.9511 & +0.0185 & -0.7 & 140 & -0.0198 & -0.0161 & +0.7872 & +0.0214 \\
\hline $20013 \leftarrow 00001 \quad(636)$ & -0.8535 & -0.70 & & -0.5860 & & 465 & +0.8449 & +0.9625 & -0.0703 & -0.7 & 680 & +0.0812 & +0.0617 & -0.8489 & -0.1110 \\
\hline $20012 \longleftarrow 00001 \quad(628)$ & -0.7941 & -0.61 & & -0.4689 & & 234 & +0.7806 & +0.9511 & +0.0185 & -0.7 & 140 & -0.0198 & -0.0161 & -0.7872 & +0.0214 \\
\hline $20013 \leftarrow 00001 \quad(628)$ & -0.8253 & -0.67 & & -0.5432 & & 385 & +0.8185 & +0.9506 & +0.0447 & -0.7 & 307 & -0.0203 & -0.0084 & -0.8187 & -0.0126 \\
\hline
\end{tabular}

Notes: The bands identified with superscripts $\left({ }^{\mathrm{a}}\right.$ and $\left.{ }^{\mathrm{b}}\right)$ are in Fermi resonance. The interaction coefficient determined in this study $=0.019295(5)$. 
${ }^{\mathrm{c}}$ The strength of this band was too small to be determined and hence fixed to the listed value after several trials during the least squares solution. Because of the presence of a very weak $Q$ branch, the $a_{4}$ term of the Herman-Wallis parameter needed adjustments and its value is determined to be: For the $21113 \leftarrow 01101$ band, $a_{4}$ (even $\left.Q\right)=+0.000177(10)$ and for the $40002 \leftarrow 100002$ band $a_{4}($ odd $Q)=-0.0054(5)$

The band centers and rotational constants are in $\mathrm{cm}^{-1}$. The rotational constants for the lower states (ground state, 01101, 10001, 10002) are from Miller and Brown [41].

$\mathrm{L}^{\prime \prime}(10001)=0.5663 \times 10^{-18} \mathrm{~cm}^{-1}$ and $\mathrm{L}^{\prime \prime}(10002)=-0.9928 \times 10^{-18} \mathrm{~cm}^{-1}$ where $\mathrm{L}^{\prime \prime}$ corresponds to the higher order (octal) term, $L^{\prime \prime}\left\{J^{\prime \prime}\left[J^{\prime \prime}+\right.\right.$ 1] $\}^{4}$, after the $H^{\prime \prime}\left\{J^{\prime \prime}\left[J^{\prime \prime}+1\right]\right\}^{3}$ term in Eq. (1), for the lower vibrational state. We did not include this term in our analysis. However, this term was determined by Miller and Brown [41] and hence we listed the L" values for completeness. Since our present study involved the lower states 10001 and 10002 in some of the bands, we listed the L" values for these two lower states. The lower level 10001 was involved in the fragmentary band $\left(30012 \leftarrow 100001\right.$ of $\left.{ }^{12} \mathrm{C}^{16} \mathrm{O}_{2}\right)$ that appeared at the upper wavenumber limit in our fitted interval with band center located at $\sim 4959.66689(5) \mathrm{cm}^{-1}$.

The intensity constants are the same for the $e$ and $f$ levels of the $21113 \leftarrow 01101$ band. The lower state rotational constants for the 636 and 628 bands were taken from Elliot et al. [42].

The vibrational band strengths $\left(S_{V}\right)$ are in $\mathrm{cm}^{-1} /\left(\right.$ molecule $\left.\cdot \mathrm{cm}^{-2}\right) \times 10^{-23}$ at $296 \mathrm{~K} \mathrm{CO}_{2}$ samples with natural abundance $\left({ }^{16} \mathrm{O}^{12} \mathrm{C}^{16} \mathrm{O}=0.9842\right)$ and high-purity ${ }^{12} \mathrm{C}^{16} \mathrm{O}_{2}$ samples with $\left(99.99 \%{ }^{12} \mathrm{C}\right.$-enriched) abundances (see text for details) were used in recording the spectra. The rotational partition sums used for ${ }^{12} \mathrm{C}^{16} \mathrm{O}_{2},{ }^{13} \mathrm{C}^{16} \mathrm{O}_{2},{ }^{12} \mathrm{C}^{16} \mathrm{O}^{18} \mathrm{O}$ at $296 \mathrm{~K}$ are from R.R. Gamache [43] and their values are: 263.87063, 527.71608, and 559.30454 , respectively. Contribution due to vibrational partition function for the lower vibrational levels was applied in computing the vibrational band strengths $\left(S_{\mathrm{v}}\right)$ listed in the Table above. 
Table 6(b)

Correlation coefficients for the upper state ro-vibrational constants for the $21113 \leftarrow 01101 e, 21113 \leftarrow 01101 f$ and the $40002 \leftarrow 10002$ interacting bands of ${ }^{12} \mathrm{C}^{16} \mathrm{O}_{2}$

\begin{tabular}{|c|c|c|c|c|c|c|c|c|c|c|c|}
\hline & \multicolumn{3}{|l|}{$21113 e$} & \multicolumn{3}{|l|}{$21113 f$} & \multicolumn{5}{|l|}{40002} \\
\hline & $B_{e}^{\prime}$ & $D_{e}^{\prime}$ & $H_{e}^{\prime}$ & $B_{f}^{\prime}$ & $D_{f}^{\prime}$ & $H_{f}^{\prime}$ & $G^{\prime}-G^{\prime \prime}$ & $B_{e}^{\prime}$ & $D_{e}^{\prime}$ & $H_{e}^{\prime}$ & $\Omega$ \\
\hline $\begin{array}{l}21113 e \\
G^{\prime}-G^{\prime \prime}\end{array}$ & -0.8159 & -0.6427 & -0.5309 & +0.3229 & +0.2518 & +0.2009 & -0.1679 & -0.0844 & -0.0277 & -0.0069 & +0.5382 \\
\hline$B_{e}^{\prime}$ & $\mathrm{X}$ & +0.9280 & +0.8306 & -0.2683 & -0.2095 & -0.1671 & +0.1321 & +0.0703 & +0.0214 & +0.0031 & -0.4441 \\
\hline$D_{e}^{\prime}$ & $\mathrm{x}$ & $\mathrm{X}$ & +0.9691 & -0.2106 & -0.1641 & -0.1306 & +0.1030 & +0.0538 & +0.0144 & -0.0005 & -0.3495 \\
\hline$H_{e}^{\prime}$ & $\mathrm{x}$ & $\mathrm{x}$ & $\mathrm{X}$ & -0.1732 & -0.1348 & -0.1071 & +0.0845 & +0.0440 & +0.0112 & -0.0012 & -0.2883 \\
\hline $\begin{array}{c}21113 \mathrm{f} \\
B_{f}^{\prime}\end{array}$ & $\mathrm{x}$ & $\mathrm{x}$ & $\mathrm{x}$ & $\mathrm{X}$ & +0.9665 & +0.8962 & +0.0372 & -0.3944 & -0.2464 & -0.1725 & +0.9458 \\
\hline$D_{f}^{\prime}$ & $\mathrm{X}$ & $\mathrm{x}$ & $\mathrm{x}$ & $\mathrm{x}$ & $\mathrm{X}$ & +0.9757 & +0.0546 & -0.4130 & -0.2912 & -0.2247 & +0.8605 \\
\hline$H_{f}^{\prime}$ & $\mathrm{X}$ & $\mathrm{x}$ & $\mathrm{X}$ & $X$ & $\mathrm{X}$ & $\mathrm{X}$ & +0.0739 & -0.4204 & -0.3281 & -0.2779 & +0.7665 \\
\hline $\begin{array}{l}40002 \\
G^{\prime}-G^{\prime \prime}\end{array}$ & $\mathrm{x}$ & $\mathrm{x}$ & $\mathrm{x}$ & $\mathrm{x}$ & $\mathrm{X}$ & $\mathrm{x}$ & $\mathrm{x}$ & -0.7773 & -0.6819 & -0.5930 & -0.0082 \\
\hline$B_{e}^{\prime}$ & $\mathrm{X}$ & $\mathrm{X}$ & $\mathrm{X}$ & $\mathrm{X}$ & $\mathrm{X}$ & $\mathrm{X}$ & $\mathrm{X}$ & $\mathrm{X}$ & +0.9487 & +0.8609 & -0.3388 \\
\hline$D_{e}^{\prime}$ & $\mathrm{x}$ & $\mathrm{x}$ & $\mathrm{x}$ & $\mathrm{X}$ & $\mathrm{x}$ & $\mathrm{x}$ & $\mathrm{x}$ & $\mathrm{x}$ & $\mathrm{X}$ & +0.9685 & -0.1820 \\
\hline$H_{e}^{\prime}$ & $\mathrm{x}$ & $\mathrm{x}$ & $\mathrm{x}$ & $\mathrm{x}$ & $\mathrm{x}$ & $\mathrm{x}$ & $\mathrm{x}$ & $\mathrm{x}$ & $\mathrm{x}$ & $\mathrm{X}$ & -0.1118 \\
\hline
\end{tabular}

Notes: The $e$ levels of $21113 \leftarrow 01101$ and the $40002 \leftarrow 01101$ bands are in Fermi resonance. The interaction parameter $\Omega$ is determined to be 0.019295(5). 
Correlation coefficients for the intensity parameters of the interacting bands $21113 \leftarrow 01101$ and the $40002 \leftarrow 10002$ bands of ${ }^{12} \mathrm{C}^{16} \mathrm{O}_{2}$

\begin{tabular}{|c|c|c|c|c|c|c|c|c|c|c|}
\hline & \multicolumn{5}{|c|}{$21113 \leftarrow 01101$} & \multicolumn{4}{|c|}{$40002 \longleftarrow 10002$} & \multirow[b]{2}{*}{$\Omega$} \\
\hline & $S_{v}$ & $a_{1}$ & $a_{2}$ & $a_{3}$ & $a_{4}$ & $a_{1}$ & $a_{2}$ & $a_{3}$ & $a_{4}$ & \\
\hline $\begin{array}{l}21113 \leftarrow 01101 \\
S_{v}\end{array}$ & $\mathrm{x}$ & -0.1183 & -0.1100 & +0.0545 & +0.1004 & +0.0155 & -0.0675 & -0.0095 & +0.0651 & -0.0126 \\
\hline$a_{1}$ & $\mathrm{X}$ & $\mathrm{X}$ & +0.9098 & -0.3407 & -0.9098 & +0.0060 & -0.0179 & -0.0166 & +0.0180 & -0.0023 \\
\hline$a_{2}$ & $\mathrm{X}$ & $\mathrm{x}$ & $\mathrm{X}$ & -0.0116 & $-0.999904^{\mathrm{a}}$ & -0.0029 & -0.0263 & +0.0003 & +0.0249 & -0.0035 \\
\hline$a_{3}$ & $\mathrm{x}$ & $\mathrm{x}$ & $\mathrm{x}$ & $\mathrm{X}$ & +0.0114 & -0.0715 & -0.0154 & +0.1410 & +0.0089 & +0.0021 \\
\hline$a_{4}$ & $\mathrm{x}$ & $\mathrm{x}$ & $\mathrm{x}$ & $\mathrm{x}$ & $\mathrm{X}$ & +0.0024 & +0.0269 & -0.0002 & -0.0254 & +0.0034 \\
\hline $\begin{array}{l}40002 \leftarrow 10002 \\
a_{1}\end{array}$ & $\mathrm{x}$ & $\mathrm{x}$ & $\mathrm{x}$ & $\mathrm{x}$ & $\mathrm{x}$ & $\mathrm{X}$ & +0.1939 & -0.8419 & -0.1993 & +0.0053 \\
\hline$a_{2}$ & $\mathrm{X}$ & $\mathrm{X}$ & $\mathrm{X}$ & $\mathrm{X}$ & $\mathrm{X}$ & $\mathrm{X}$ & $\mathrm{X}$ & +0.0012 & -0.0059 & +0.0060 \\
\hline$a_{3}$ & $\mathrm{x}$ & $\mathrm{x}$ & $\mathrm{x}$ & $\mathrm{x}$ & $\mathrm{x}$ & $\mathrm{x}$ & $\mathrm{x}$ & $\mathrm{X}$ & -0.0014 & -0.0059 \\
\hline$a_{4}$ & $\mathrm{x}$ & $\mathrm{x}$ & $\mathrm{x}$ & $\mathrm{x}$ & $\mathrm{x}$ & $\mathrm{x}$ & $\mathrm{x}$ & $\mathrm{x}$ & $\mathrm{X}$ & -0.0080 \\
\hline$\Omega$ & $\mathrm{x}$ & $\mathrm{x}$ & $\mathrm{x}$ & $\mathrm{x}$ & $\mathrm{x}$ & $\mathrm{X}$ & $\mathrm{X}$ & $\mathrm{x}$ & $\mathrm{x}$ & $\mathrm{X}$ \\
\hline
\end{tabular}

Notes: Although separate ro-vibrational constants $(B, D, H)$ were determined for the $e$ and the $f$ levels for the $21113 \leftarrow 01101$ band, the band strength and the Herman-Wallis parameters could not be determined for each level and hence only one value for both levels were determined (see Table 6(a)). The off-diagonal terms marked $\mathrm{x}$ are symmetric with those listed above the diagonal.

${ }^{a}$ Due to the very large correlation between the $a_{2}$ and the $a_{4}$ terms, $(21113 \leftarrow 01101$ band), more digits are provided to avoid any problems that could arise due to truncation while computing the error analysis. 


\section{Table 7}

Comparisons of vibrational band strengths, $S_{\mathrm{v}}$, $(\mathrm{cm} /$ molecule at $296 \mathrm{~K})$ for select bands ${ }^{\mathrm{a}}$ measured in the $2-\mu \mathrm{m}$ region of $\mathrm{CO}_{2}$.

\begin{tabular}{|c|c|c|c|c|c|c|}
\hline \multirow[t]{2}{*}{ Iso. } & \multirow{2}{*}{$\begin{array}{l}\text { Band } \\
\text { er } \leftarrow \text { Lower }\end{array}$} & \multirow{2}{*}{$\begin{array}{l}\text { Band } \\
\text { center } \\
\left(\mathrm{cm}^{-1}\right)^{b}\end{array}$} & \multicolumn{2}{|c|}{$\begin{array}{l}\text { Vibrational Band strength } \\
\qquad\left(S_{\mathrm{v}}\right)\end{array}$} & \multicolumn{2}{|c|}{$\#$ lines and range in $J$} \\
\hline & & & PS & Toth et al. & PS & Toth et al. \\
\hline 626 & $21113 \leftarrow 01101$ & 4807.694 & $6.5488(6) \times 10^{-22}$ & $6.558(10) \times 10^{-22}$ & 183; (P73-R72) & 258;(P95-R96) \\
\hline 626 & $40002 \leftarrow 01101^{\mathrm{c}}$ & 4808.174 & $\begin{array}{l}1.23 \times 10^{-26} \\
\text { (Fixed) }\end{array}$ & $5.24(72) \times 10^{-26}$ & $\begin{array}{c}65 ;(\mathrm{P} 55- \\
\mathrm{R} 55 ; \mathrm{Q} 2-\mathrm{Q} 20)\end{array}$ & 40; (P51-R53) \\
\hline 626 & $20013 \leftarrow 00001$ & 4853.623 & $6.88420(2) \times 10^{-21}$ & $6.897(6) \times 10^{-21}$ & 85; (P84-R84) & 105; (P104-R104) \\
\hline 626 & $30013 \leftarrow 10002^{\mathrm{d}}$ & 4942.508 & $1.123(1) \times 10^{-22}$ & $1.277(2) \times 10^{-22}$ & 29; (P68-P12) & 93; (P92-R92) \\
\hline 626 & $21112 \leftarrow 01101^{\mathrm{d}}$ & 4965.385 & $1.686(2) \times 10^{-21}$ & $4.73 \times 10^{-21}$ & 42; (P77-P36) & 268; (P98-R98) \\
\hline 626 & $30014 \leftarrow 10002$ & 4790.572 & $3.331(4) \times 10^{-23}$ & $3.346 \times 10^{-23}$ & 65; (P64-R64) & 89; (P88-R88) \\
\hline 636 & $20012 \leftarrow 00001^{\mathrm{e}}$ & 4887.385 & $2.6690(8) \times 10^{-22}$ & $2.61 \times 10^{-22}$ & 73; (P72-R72) & 95; (P94-R94) \\
\hline 636 & $20013 \leftarrow 00001^{\mathrm{e}}$ & 4848.066 & $2.7080(2) \times 10^{-23}$ & $2.72 \times 10^{-23}$ & 82; (P60-R64) & 89; (P88-R88) \\
\hline 628 & $20012 \leftarrow 00001^{f}$ & 4904.859 & $1.2988(6) \times 10^{-22}$ & $1.25 \times 10^{-22}$ & 117; (P67-R49) & 185; (P92-R92) \\
\hline 628 & $20013 \leftarrow 00001^{\mathrm{f}}$ & 4791.259 & $4.8692(4) \times 10^{-23}$ & $4.65 \times 10^{-23}$ & 129; P(64-R64) & 182; (P90-R91) \\
\hline
\end{tabular}

${ }^{a}$ Only those bands whose Vibrational band strengths $\left(S_{\mathrm{v}}\right)$ have been determined using theoretical quantum mechanical expressions (Eqs.1-3) are listed in this Table. For other bands (including a number of weaker bands) individual line positions and intensities were adjusted in the least squares fits when appropriate, and their values are given in the Supplemental files.

${ }^{\mathrm{b}}$ For purpose of identification only.

c The band was weak and its vibrational band strength was fixed in the least squares fit (see Table 6(a)).

${ }^{\mathrm{d}}$ Transitions from only $\mathrm{P}$ branch were observed in the present study.

${ }^{\mathrm{e}}$ Only two ${ }^{13} \mathrm{C}$-enriched spectra were included in the fit (see the text for details).

${ }^{\mathrm{f}}$ One spectrum with natural $\mathrm{CO}_{2}$ sample was included in the fit. All other spectra (including airbroadened) contained $99.99 \%{ }^{12} \mathrm{C}$-enhancement where abundances of rarer isotopologues (e.g., $\left.{ }^{13} \mathrm{C}^{16} \mathrm{O}_{2},{ }^{12} \mathrm{C}^{16} \mathrm{O}^{18} \mathrm{O},{ }^{12} \mathrm{C}^{16} \mathrm{O}^{18} \mathrm{O}\right)$ in each spectrum were determined. The differences observed between PS and Toth et al. $[\mathbf{1 1 , 1 5}]$ reflect those estimates and should be considered as only estimated vibrational band strengths. Because of the sample purity used, the intensities for 636 and 628 bands listed in $[\mathbf{1 1}, \mathbf{1 5}]$ should be more accurate than from present study. 
Table 8

A sample of measured line parameters for the P20-R20 transitions in the $20013 \leftarrow 00001$ band of ${ }^{12} \mathrm{C}^{16} \mathrm{O}_{2}$.

\begin{tabular}{|c|c|c|c|c|c|c|c|c|c|c|c|}
\hline Line & $\begin{array}{l}\text { Position and } \\
\text { unc. }^{\text {a }}\end{array}$ & Intensity $S^{b}$ & $\begin{array}{l}\mathrm{S} \\
(\% \text { unc })\end{array}$ & $\begin{array}{l}\text { Lorentz half- } \\
\text { width } \\
\text { coefficients }^{c}\end{array}$ & $\begin{array}{l}\% \\
\text { unc }\end{array}$ & $\begin{array}{l}n_{1} \text { and } \\
n_{2}{ }^{\mathrm{d}}\end{array}$ & $\%$ unc & $\begin{array}{l}\text { Pressure shift } \\
\left(\delta^{0}\right) \text { and unc. }\end{array}$ & $\begin{array}{l}\text { T-dep. pressure } \\
\text { shift }\left(\delta^{\prime}\right) \text { and } \\
\text { unc. }\end{array}$ & $\begin{array}{l}\text { Speed } \\
\text { dependence }^{f}\end{array}$ & $\%$ unc \\
\hline P20 & 4837.243864( 1) & $2.376 \times 10^{-22}$ & 0.003 & $\begin{array}{l}0.07341 \\
0.10032\end{array}$ & $\begin{array}{l}0.01 \\
0.06\end{array}$ & $\begin{array}{l}0.7353 \\
0.6616\end{array}$ & $\begin{array}{l}0.08 \\
0.21\end{array}$ & $\begin{array}{l}-0.005287(12) \\
-0.006792(102)\end{array}$ & $\begin{array}{l}+0.0198(22) \\
-0.2100(122)\end{array}$ & 0.1061 & 0.5 \\
\hline P18 & $4838.955594(1)$ & $2.471 \times 10^{-22}$ & 0.003 & $\begin{array}{l}0.07462 \\
0.10223\end{array}$ & $\begin{array}{l}0.01 \\
0.06\end{array}$ & $\begin{array}{l}0.7257 \\
0.6736\end{array}$ & $\begin{array}{l}0.08 \\
0.19\end{array}$ & $\begin{array}{l}-0.005203(12) \\
-0.006761(100)\end{array}$ & $\begin{array}{l}+0.0151(22) \\
-0.2350(118)\end{array}$ & 0.1125 & 0.5 \\
\hline P16 & $4840.650679(1)$ & $2.502 \times 10^{-22}$ & 0.003 & $\begin{array}{l}0.07599 \\
0.10396\end{array}$ & $\begin{array}{l}0.01 \\
0.06\end{array}$ & $\begin{array}{l}0.7202 \\
0.6846\end{array}$ & $\begin{array}{l}0.08 \\
0.19\end{array}$ & $\begin{array}{l}-0.005171(12) \\
-0.006627(99)\end{array}$ & $\begin{array}{l}+0.0082(22) \\
-0.2342(115)\end{array}$ & 0.1178 & 0.4 \\
\hline P14 & $4842.329244(1)$ & $2.456 \times 10^{-22}$ & 0.003 & $\begin{array}{l}0.07754 \\
0.10581\end{array}$ & $\begin{array}{l}0.03 \\
0.06\end{array}$ & $\begin{array}{l}0.7155 \\
0.6909\end{array}$ & $\begin{array}{l}0.08 \\
0.19\end{array}$ & $\begin{array}{l}-0.005016(12) \\
-0.006418(100)\end{array}$ & $\begin{array}{l}+0.0013(22) \\
-0.2492(115)\end{array}$ & 0.1224 & 0.4 \\
\hline P12 & $4843.991396(1)$ & $2.328 \times 10^{-22}$ & 0.003 & $\begin{array}{l}0.07922 \\
0.10808\end{array}$ & $\begin{array}{l}0.03 \\
0.06\end{array}$ & $\begin{array}{l}0.7135 \\
0.6891\end{array}$ & $\begin{array}{l}0.08 \\
0.19\end{array}$ & $\begin{array}{l}-0.004811(12) \\
-0.006155(103)\end{array}$ & $\begin{array}{l}-0.0110(23) \\
-0.2434(117)\end{array}$ & 0.1250 & 0.4 \\
\hline P10 & $4845.637224(1)$ & $2.114 \times 10^{-22}$ & 0.003 & $\begin{array}{l}0.08109 \\
0.11061\end{array}$ & $\begin{array}{l}0.02 \\
0.06\end{array}$ & $\begin{array}{l}0.7134 \\
0.6886\end{array}$ & $\begin{array}{l}0.08 \\
0.19\end{array}$ & $\begin{array}{l}-0.004546(12) \\
-0.006286(110)\end{array}$ & $\begin{array}{l}-0.0205(23) \\
-0.2901(124)\end{array}$ & 0.1260 & 0.4 \\
\hline P8 & $4847.266801(1)$ & $1.816 \times 10^{-22}$ & 0.003 & $\begin{array}{l}0.08302 \\
0.11340\end{array}$ & $\begin{array}{l}0.02 \\
0.06\end{array}$ & $\begin{array}{l}0.7169 \\
0.6903\end{array}$ & $\begin{array}{l}0.08 \\
0.20\end{array}$ & $\begin{array}{l}-0.004281(14) \\
-0.006199(120)\end{array}$ & $\begin{array}{l}-0.0394(24) \\
-0.3165(134)\end{array}$ & 0.1231 & 0.4 \\
\hline P6 & $4848.880178(1)$ & $1.441 \times 10^{-22}$ & 0.003 & $\begin{array}{l}0.08505 \\
0.11641\end{array}$ & $\begin{array}{l}0.02 \\
0.07\end{array}$ & $\begin{array}{l}0.7237 \\
0.6988\end{array}$ & $\begin{array}{l}0.10 \\
0.23\end{array}$ & $\begin{array}{l}-0.003941(15) \\
-0.006380(138)\end{array}$ & $\begin{array}{l}-0.0504(26) \\
-0.3529(153)\end{array}$ & 0.1159 & 0.5 \\
\hline $\mathrm{P} 4$ & $4850.477391(1)$ & $1.002 \times 10^{-22}$ & 0.003 & $\begin{array}{l}0.08735 \\
0.11928\end{array}$ & $\begin{array}{l}0.02 \\
0.08\end{array}$ & $\begin{array}{l}0.7275 \\
0.7195\end{array}$ & $\begin{array}{l}0.14 \\
0.25\end{array}$ & $\begin{array}{l}-0.003495(16) \\
-0.006324(151)\end{array}$ & $\begin{array}{l}-0.0618(24) \\
-0.3935(163)\end{array}$ & 0.1031 & 0.7 \\
\hline P2 & $4852.058456(1)$ & $5.146 \times 10^{-22}$ & 0.003 & $\begin{array}{l}0.09099 \\
0.12319\end{array}$ & $\begin{array}{l}0.03 \\
0.11\end{array}$ & $\begin{array}{l}0.7210 \\
0.7447\end{array}$ & $\begin{array}{l}0.19 \\
0.32\end{array}$ & $\begin{array}{l}-0.002959(22) \\
-0.006038(217)\end{array}$ & $\begin{array}{l}-0.1046(34) \\
-0.4302(224)\end{array}$ & 0.0843 & 1.3 \\
\hline R0 & $4854.399765(1)$ & $2.607 \times 10^{-22}$ & 0.003 & $\begin{array}{l}0.09529 \\
0.12964\end{array}$ & $\begin{array}{l}0.05 \\
0.16\end{array}$ & $\begin{array}{l}0.7116 \\
0.7581\end{array}$ & $\begin{array}{l}0.20 \\
0.44\end{array}$ & $\begin{array}{l}-0.002882(37) \\
-0.005635(291)\end{array}$ & $\begin{array}{l}-0.0682(62) \\
-0.2994(272)\end{array}$ & 0.0558 & 4.1 \\
\hline $\mathrm{R} 2$ & $4855.940408(1)$ & $7.746 \times 10^{-22}$ & 0.003 & $\begin{array}{l}0.08826 \\
0.12011\end{array}$ & $\begin{array}{l}0.02 \\
0.09\end{array}$ & $\begin{array}{l}0.7251 \\
0.7414\end{array}$ & $\begin{array}{l}0.11 \\
0.27\end{array}$ & $\begin{array}{l}-0.003330(20) \\
-0.006366(174)\end{array}$ & $\begin{array}{l}-0.0756(33) \\
-0.3900(185)\end{array}$ & 0.0757 & 1.2 \\
\hline $\mathrm{R} 4$ & $4857.464813(1)$ & $1.260 \times 10^{-22}$ & 0.003 & $\begin{array}{l}0.08578 \\
0.11703\end{array}$ & $\begin{array}{l}0.02 \\
0.07\end{array}$ & $\begin{array}{l}0.7204 \\
0.7173\end{array}$ & $\begin{array}{l}0.10 \\
0.22\end{array}$ & $\begin{array}{l}-0.003503(16) \\
-0.006172(149)\end{array}$ & $\begin{array}{l}-0.0902(27) \\
-0.4195(165)\end{array}$ & 0.0951 & 0.7 \\
\hline R6 & $4858.972913(1)$ & $1.696 \times 10^{-22}$ & 0.003 & $\begin{array}{l}0.08378 \\
0.11419\end{array}$ & $\begin{array}{l}0.02 \\
0.07\end{array}$ & $\begin{array}{l}0.7129 \\
0.7014\end{array}$ & $\begin{array}{l}0.10 \\
0.21\end{array}$ & $\begin{array}{l}-0.003608(14) \\
-0.005814(128)\end{array}$ & $\begin{array}{l}-0.0985(25) \\
-0.3453(141)\end{array}$ & 0.1128 & 0.5 \\
\hline R8 & $4860.464622(1)$ & $2.067 \times 10^{-22}$ & 0.003 & 0.08182 & 0.02 & 0.7061 & 0.09 & $-0.003655(13)$ & $-0.1059(23)$ & 0.1226 & 0.4 \\
\hline
\end{tabular}




\begin{tabular}{|c|c|c|c|c|c|c|c|c|c|c|c|}
\hline & & & & 0.11150 & 0.06 & 0.6907 & 0.19 & $-0.006118(112)$ & $-0.3410(125)$ & & \\
\hline R10 & 4861.939835( 1) & $2.359 \times 10^{-22}$ & 0.003 & $\begin{array}{l}0.07978 \\
0.10876\end{array}$ & $\begin{array}{l}0.03 \\
0.06\end{array}$ & $\begin{array}{l}0.7053 \\
0.6917\end{array}$ & $\begin{array}{l}0.09 \\
0.19\end{array}$ & $\begin{array}{l}-0.003685(13) \\
-0.006188(103)\end{array}$ & $\begin{array}{l}-0.0977(23) \\
-0.3093(116)\end{array}$ & 0.1248 & 0.4 \\
\hline R12 & $4863.398430(1)$ & $2.566 \times 10^{-22}$ & 0.003 & $\begin{array}{l}0.07799 \\
0.10641\end{array}$ & $\begin{array}{l}0.03 \\
0.06\end{array}$ & $\begin{array}{l}0.7045 \\
0.6916\end{array}$ & $\begin{array}{l}0.09 \\
0.19\end{array}$ & $\begin{array}{l}-0.003880(12) \\
-0.006121(99)\end{array}$ & $\begin{array}{l}-0.0979(22) \\
-0.2675(113)\end{array}$ & 0.1246 & 0.4 \\
\hline R14 & $4864.840267(1)$ & $2.685 \times 10^{-22}$ & 0.003 & $\begin{array}{l}0.07628 \\
0.10435\end{array}$ & $\begin{array}{l}0.01 \\
0.06\end{array}$ & $\begin{array}{l}0.7095 \\
0.6889\end{array}$ & $\begin{array}{l}0.08 \\
0.19\end{array}$ & $\begin{array}{l}-0.004025(12) \\
-0.006319(96)\end{array}$ & $\begin{array}{l}-0.0886(22) \\
-0.2596(130)\end{array}$ & 0.1214 & 0.4 \\
\hline R16 & $4866.265187(1)$ & $2.718 \times 10^{-22}$ & 0.003 & $\begin{array}{l}0.07483 \\
0.10262\end{array}$ & $\begin{array}{l}0.01 \\
0.06\end{array}$ & $\begin{array}{l}0.7147 \\
0.6815\end{array}$ & $\begin{array}{l}0.08 \\
0.19\end{array}$ & $\begin{array}{l}-0.004181(12) \\
-0.006534(96)\end{array}$ & $\begin{array}{l}-0.0804(22) \\
-0.2351(111)\end{array}$ & 0.1188 & 0.4 \\
\hline R18 & 4867.673013( 1) & $2.675 \times 10^{-22}$ & 0.003 & $\begin{array}{l}0.07360 \\
0.10111\end{array}$ & $\begin{array}{l}0.01 \\
0.05\end{array}$ & $\begin{array}{l}0.7231 \\
0.6658\end{array}$ & $\begin{array}{l}0.08 \\
0.20\end{array}$ & $\begin{array}{l}-0.004368(12) \\
-0.006665(95)\end{array}$ & $\begin{array}{l}-0.0718(22) \\
-0.2059(112)\end{array}$ & 0.1147 & 0.5 \\
\hline R20 & 4869.063551( 1) & $2.566 \times 10^{-22}$ & 0.003 & $\begin{array}{l}0.07251 \\
0.09920\end{array}$ & $\begin{array}{l}0.01 \\
0.06\end{array}$ & $\begin{array}{l}0.7344 \\
0.6557\end{array}$ & $\begin{array}{l}0.08 \\
0.21\end{array}$ & $\begin{array}{l}-0.004529(12) \\
-0.006792(98)\end{array}$ & $\begin{array}{l}-0.0656(22) \\
-0.1828(116)\end{array}$ & 0.1096 & 0.5 \\
\hline
\end{tabular}

The percent $(\%)$ uncertainties listed under various columns correspond to one sigma internal statistical errors obtained from least-squares fits.

${ }^{a}$ Positions and uncertainties are in $\mathrm{cm}^{-1}$.

${ }^{\mathrm{b}}$ Line intensities $(S)$ are in $\mathrm{cm} / \mathrm{molecule}$ at $296 \mathrm{~K}$.

${ }^{\mathrm{c}}$ Lorentz half-width coefficients $\left(b_{L}^{0}\right)$ and pressureshift coefficients $\left(\delta^{0}\right)$ are in units $\mathrm{of} \mathrm{cm}^{-1} \mathrm{~atm}^{-1}$ at $296 \mathrm{~K}$. The numbers in the top row in each column correspond to air-broadening and the bottom self-broadening.

${ }^{\mathrm{d}} n_{1}$ and $n_{2}$ are the temperature dependence exponents of air- and self-width coefficients, respectively, and have no units.

${ }^{\mathrm{e}}$ Temperature dependences of pressure shift coefficients, $\delta^{\prime}$ (air) and $\delta^{\prime}$ (self), are in units of $\mathrm{cm}^{-1} \mathrm{~atm}^{-1} \mathrm{~K}^{-1}$.

${ }^{\mathrm{f}}$ Quadratic speed dependence parameter has no units. 


\section{Table 9}

Measured off-diagonal relaxation matrix element coefficients, $W_{\mathrm{ij}}$, $\left(\mathrm{cm}^{-1} \mathrm{~atm}^{-1}\right.$ at $\left.296 \mathrm{~K}\right)$, and their temperature dependence exponents (unitless) for $\mathrm{CO}_{2}-\mathrm{CO}_{2}$ and $\mathrm{CO}_{2}$-air for the $20013 \leftarrow 00001$ band of ${ }^{12} \mathrm{C}^{16} \mathrm{O}_{2}$.

\begin{tabular}{|c|c|c|c|c|}
\hline $\begin{array}{l}\text { Mixing } \\
\text { between }\end{array}$ & $\begin{array}{l}\mathrm{RMEC}\left(\mathrm{CO}_{2^{-}}\right. \\
\left.\mathrm{CO}_{2}\right) \\
\left(W_{\mathrm{ij}}\right) \text { at } 296 \mathrm{~K}\end{array}$ & $\begin{array}{l}\text { Temp. dep. } \\
\text { Exponents for } \\
\left(\mathrm{CO}_{2}-\mathrm{CO}_{2}\right)\end{array}$ & $\begin{array}{l}\text { RMEC } \\
\left(\mathrm{CO}_{2} \text {-air }\right) \\
\left(W_{\mathrm{ij}}\right) \text { at } 296 \mathrm{~K}\end{array}$ & $\begin{array}{l}\text { Temp. dep. } \\
\text { exponents for } \\
\mathrm{CO}_{2} \text {-air }\end{array}$ \\
\hline $\mathrm{P} 2 \& \mathrm{P} 4$ & $0.00471(32)$ & $0.80 f x d$ & $0.00637(5)$ & $0.80 f x d$ \\
\hline P4 \& P6 & $0.00830(33)$ & $0.80 \mathrm{fxd}$ & $0.01107(5)$ & $0.80 \mathrm{fxd}$ \\
\hline P6 \& P8 & $0.01135(43)$ & $0.958(81)$ & $0.01450(6)$ & $0.860(12)$ \\
\hline P8 \&P 10 & $0.01427(47)$ & $1.060(77)$ & $0.01703(7)$ & $0.892(13)$ \\
\hline $\mathrm{P} 10 \& \mathrm{P} 12$ & $0.01714(50)$ & $1.104(71)$ & $0.01896(7)$ & $0.917(13)$ \\
\hline $\mathrm{P} 12$ \&P 14 & $0.02092(54)$ & $0.975(66)$ & $0.02024(7)$ & $0.948(13)$ \\
\hline P14 \& P16 & $0.02463(59)$ & $0.870(64)$ & $0.02111(8)$ & $0.948(13)$ \\
\hline P16 \& P18 & $0.02674(63)$ & $0.850(65)$ & $0.02162(9)$ & $0.934(14)$ \\
\hline P18 \& P20 & $0.02789(69)$ & 0.809 (67) & $0.02206(9)$ & $0.881(15)$ \\
\hline P20 \& P22 & $0.02904(69)$ & $0.846(70)$ & $0.02226(10)$ & $0.766(17)$ \\
\hline P22 \& P24 & $0.03056(76)$ & $0.780(71)$ & $0.02221(11)$ & $0.696(18)$ \\
\hline P24 \&P 26 & $0.03428(84)$ & $0.778(73)$ & $0.02193(13)$ & $0.599(20)$ \\
\hline P26 \& P28 & $0.03673(93)$ & $0.647(78)$ & $0.02146(14)$ & $0.556(21)$ \\
\hline P28 \& P30 & $0.04033(103)$ & $0.629(75)$ & $0.02084(15)$ & 0.623 (19) \\
\hline P30 \& P32 & $0.04295(114)$ & $0.580(64)$ & $0.01967(17)$ & $0.70 \mathrm{fxd}$ \\
\hline P32 \& P34 & 0.04451 (119) & $0.80 f x d$ & $0.01841(20)$ & $0.70 \mathrm{fxd}$ \\
\hline P34 \& P36 & $0.04412(123)$ & $0.80 f x d$ & $0.01785(23)$ & $0.70 f x d$ \\
\hline P36 \& P38 & $0.04697(140)$ & $0.80 f x d$ & $0.01663(27)$ & $0.70 f x d$ \\
\hline P38 \& P40 & $0.04270(163)$ & $0.80 f x d$ & $0.01579(33)$ & $0.70 f x d$ \\
\hline $\mathrm{P} 40$ \& P42 & $0.03890(190)$ & $0.70 f x d$ & $0.01431(38)$ & $0.70 f x d$ \\
\hline P42 \& P44 & $0.03162(217)$ & $0.70 f x d$ & $0.01324(45)$ & $0.70 f x d$ \\
\hline P44 \& P46 & $0.02225(242)$ & $0.70 f x d$ & $0.01132(51)$ & $0.70 f x d$ \\
\hline P46 \& P48 & $0.01363(254)$ & $0.70 f x d$ & $0.00940(56)$ & $0.70 \mathrm{fxd}$ \\
\hline P48 \& P50 & $0.00810(269)$ & $0.70 \mathrm{fxd}$ & $0.00470(51)$ & $0.70 f x d$ \\
\hline P50 \& P52 & $0.005 f x d$ & $0.70 f x d$ & 0.005 fxd & $0.70 f x d$ \\
\hline P52 \& P54 & $0.005 f x d$ & $0.70 \mathrm{fxd}$ & $0.005 f x d$ & $0.70 f x d$ \\
\hline P54 \& P56 & $0.005 f x d$ & 0.70 fxd & $0.005 f x d$ & $0.70 f x d$ \\
\hline P56 \& P58 & $0.005 f x d$ & $0.70 f x d$ & $0.005 f x d$ & $0.70 f x d$ \\
\hline P58 \& P60 & $0.005 f x d$ & $0.70 f x d$ & $0.005 f x d$ & $0.70 f x d$ \\
\hline R2 \& R4 & $0.00429(22)$ & $0.80 f x d$ & $0.00253(5)$ & $1.126(63)$ \\
\hline R4 \& R6 & $0.01554(32)$ & $0.80 f x d$ & $0.00997(7)$ & $0.959(25)$ \\
\hline R6 \& R8 & $0.02065(41)$ & $0.876(44)$ & $0.01453(7)$ & $0.962(17)$ \\
\hline R8 \& R10 & $0.02344(43)$ & $0.998(44)$ & $0.01763(7)$ & $0.975(14)$ \\
\hline R10 \& R12 & $0.02596(44)$ & $1.003(42)$ & $0.01986(6)$ & $0.965(12)$ \\
\hline
\end{tabular}




\begin{tabular}{|l|l|l|l|l|}
\hline R12 \& R14 & $0.02744(44)$ & $0.981(42)$ & $0.02114(7)$ & $0.981(11)$ \\
\hline R14 \& R16 & $0.02834(45)$ & $0.939(43)$ & $0.02197(7)$ & $0.981(11)$ \\
\hline R16 \& R18 & $0.02802(46)$ & $0.945(45)$ & $0.02245(7)$ & $0.969(11)$ \\
\hline R18 \& R20 & $0.02757(47)$ & $0.930(48)$ & $0.02260(7)$ & $0.949(11)$ \\
\hline R20 \& R22 & $0.02679(49)$ & $0.917(51)$ & $0.02254(7)$ & $0.931(11)$ \\
\hline R22 \& R24 & $0.02526(51)$ & $0.879(57)$ & $0.02227(7)$ & $0.877(12)$ \\
\hline R24 \& R26 & $0.02314(53)$ & $0.785(66)$ & $0.02173(8)$ & $0.826(12)$ \\
\hline R26 \& R28 & $0.02133(54)$ & $0.627(74)$ & $0.02097(8)$ & $0.780(12)$ \\
\hline R28 \& R30 & $0.01855(54)$ & $0.557(75)$ & $0.01995(8)$ & $0.737(11)$ \\
\hline R30 \& R32 & $0.01560(49)$ & $0.70 f x d$ & $0.01905(8)$ & $0.70 f x d$ \\
\hline R32 \& R34 & $0.01131(50)$ & $0.70 f x d$ & $0.01800(9)$ & $0.70 f x d$ \\
\hline R34 \& R36 & $0.00794(50)$ & $0.70 f x d$ & $0.01686(10)$ & $0.70 f x d$ \\
\hline R36 \& R38 & $0.00602(44)$ & $0.70 f x d$ & $0.01573(11)$ & $0.70 f x d$ \\
\hline R38 \& R40 & $0.004 f x d$ & $0.70 f x d$ & $0.01437(12)$ & $0.70 f x d$ \\
\hline R40 \& R42 & $0.004 f x d$ & $0.70 f x d$ & $0.01280(13)$ & $0.70 f x d$ \\
\hline R42 \& R44 & $0.004 f x d$ & $0.70 f x d$ & $0.01036(13)$ & $0.70 f x d$ \\
\hline R44 \& R46 & $0.004 f x d$ & $0.70 f x d$ & $0.00739(12)$ & $0.70 f x d$ \\
\hline R46 \& R48 & $0.004 f x d$ & $0.70 f x d$ & $0.004 f x d$ & $0.70 f x d$ \\
\hline R48 \& R50 & $0.004 f x d$ & $0.70 f x d$ & $0.004 f x d$ & $0.70 f x d$ \\
\hline R50 \& R52 & $0.004 f x d$ & $0.70 f x d$ & $0.004 f x d$ & $0.70 f x d$ \\
\hline R52 \& R54 & $0.004 f x d$ & $0.70 f x d$ & $0.004 f x d$ & $0.70 f x d$ \\
\hline
\end{tabular}

Measured off-diagonal relaxation matrix element coefficients, $W_{\mathrm{ij}}$, for $\mathrm{CO}_{2}-\mathrm{CO}_{2}$ and $\mathrm{CO}_{2}$-air for the $Q$ branch lines in the $21113 \longleftarrow 01101$ band of ${ }^{12} \mathrm{C}^{16} \mathrm{O}_{2}$.

\begin{tabular}{|c|l|l|l|c|}
\hline $\begin{array}{l}\text { Mixing } \\
\text { between }\end{array}$ & $\begin{array}{l}\text { RMEC } \\
\text { CO2-CO2 }\left(W_{\mathrm{ij}}\right) \\
\text { at 296 K }\end{array}$ & $\begin{array}{l}\text { Temperature } \\
\text { dependence } \\
\text { exponent, Self- } \\
\text { mixing }\end{array}$ & $\begin{array}{l}\text { RMEC CO2- } \\
\text { air, }\left(W_{\mathrm{ij}}\right) \text { at 296 }\end{array}$ & $\begin{array}{l}\text { Temperature } \\
\text { dependence } \\
\text { exponent, air } \\
\left.\text { mixing } W_{\mathrm{ij}}\right)\end{array}$ \\
\hline Q1 \& Q3 & $0.00527(59)$ & $0.70 f x d$ & $0.00090(9)$ & $0.70 f x d$ \\
\hline Q3 \& Q2 & $0.00471(92)$ & $0.70 f x d$ & $0.00228(24)$ & $0.70 f x d$ \\
\hline Q2 \& Q5 & $0.00464(87)$ & $0.70 f x d$ & $0.00293(28)$ & $0.70 f x d$ \\
\hline Q5 \& Q7 & $0.02636(284)$ & $0.70 f x d$ & $0.01803(54)$ & $0.70 f x d$ \\
\hline Q7 \& Q4 & $0.0010(223)$ & $0.70 f x d$ & $0.01877(67)$ & $0.70 f x d$ \\
\hline Q4 \& Q9 & $0.0042(16)$ & $0.70 f x d$ & $0.00863(44)$ & $0.70 f x d$ \\
\hline Q9 \& Q6 & $0.01175(410)$ & $0.70 f x d$ & $0.02363(65)$ & $0.70 f x d$ \\
\hline Q6 \& Q11 & $0.00723(42)$ & $0.70 f x d$ & $0.00358(38)$ & $0.70 f x d$ \\
\hline Q11 \& Q13 & $0.005 f x d$ & $0.70 f x d$ & $0.005 f x d$ & $0.70 f x d$ \\
\hline Q13 \& Q8 & $0.005 f x d$ & $0.70 f x d$ & $0.005 f x d$ & $0.70 f x d$ \\
\hline
\end{tabular}

The relaxation matrix element coefficients for only the $Q$ lines were measured in this band. These parameter values could not be measured accurately; nevertheless needed adjustments to minimize the fit residuals. The relaxation matrix element coefficients for the nearest neighbor $Q$ lines only were considered (regardless of their assignments) in this analysis. 